\title{
Hard Squares with Negative Activity and Rhombus Tilings of the Plane
}

\author{
Jakob Jonsson* \\ Department of Mathematics \\ Massachusetts Institute of Technology, Cambridge, MA 02139 \\ jakob@math.mit.edu
}

Submitted: Mar 24, 2006; Accepted: Jul 28, 2006; Published: Aug 7, 2006

Mathematics Subject Classifications: 05A15, 05C69, 52C20

\begin{abstract}
Let $S_{m, n}$ be the graph on the vertex set $\mathbb{Z}_{m} \times \mathbb{Z}_{n}$ in which there is an edge between $(a, b)$ and $(c, d)$ if and only if either $(a, b)=(c, d \pm 1)$ or $(a, b)=(c \pm 1, d)$ modulo $(m, n)$. We present a formula for the Euler characteristic of the simplicial complex $\Sigma_{m, n}$ of independent sets in $S_{m, n}$. In particular, we show that the unreduced Euler characteristic of $\Sigma_{m, n}$ vanishes whenever $m$ and $n$ are coprime, thereby settling a conjecture in statistical mechanics due to Fendley, Schoutens and van Eerten. For general $m$ and $n$, we relate the Euler characteristic of $\Sigma_{m, n}$ to certain periodic rhombus tilings of the plane. Using this correspondence, we settle another conjecture due to Fendley et al., which states that all roots of $\operatorname{det}\left(x I-T_{m}\right)$ are roots of unity, where $T_{m}$ is a certain transfer matrix associated to $\left\{\Sigma_{m, n}: n \geq 1\right\}$. In the language of statistical mechanics, the reduced Euler characteristic of $\Sigma_{m, n}$ coincides with minus the partition function of the corresponding hard square model with activity -1 .
\end{abstract}

\section{Introduction}

An independent set in a simple and loopless graph $G$ is a subset of the vertex set of $G$ with the property that no two vertices in the subset are adjacent. The family of independent sets in $G$ forms a simplicial complex, the independence complex $\Sigma(G)$ of $G$.

The purpose of this paper is to analyze the independence complex of square grids with periodic boundary conditions. Specifically, define $S_{m, n}$ to be the graph with vertex set $\mathbb{Z}_{m} \times \mathbb{Z}_{n}$ and with an edge between $(a, b)$ and $(c, d)$ if and only if either $(a, b)=(c, d \pm 1)$ or $(a, b)=(c \pm 1, d)$ (computations carried out modulo $(m, n))$. Defining $\mathbb{L}_{m, n}:=m \mathbb{Z} \times n \mathbb{Z}$,

\footnotetext{
* Research supported by the European Graduate Program "Combinatorics, Geometry, and Computation", DFG-GRK 588/2.
} 
we may identify the vertex set of $S_{m, n}$ with the quotient group $\mathbb{Z}^{2} / \mathbb{L}_{m, n}$. In particular, we may refer to vertices of $S_{m, n}$ as cosets of $\mathbb{L}_{m, n}$ in $\mathbb{Z}^{2}$.

To avoid misconceptions, we state already at this point that we label elements in $\mathbb{Z}^{2}$ according to the matrix convention; $(i, j)$ is the element in the $i$ th row below row 0 and the $j$ th column to the right of column 0 .

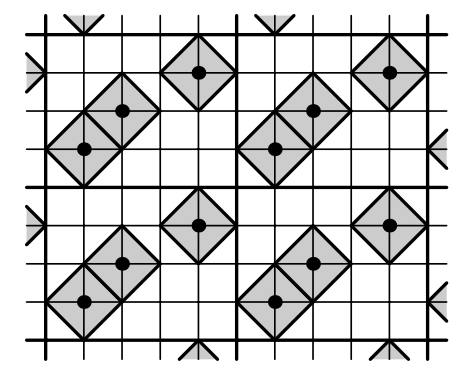

Figure 1: Configuration of hard squares invariant under translation with the vectors $(4,0)$ and $(0,5)$. The corresponding member of $\Sigma_{4,5}$ is the set of cosets of $\mathbb{L}_{4,5}$ containing the square centers.

Properties of $\Sigma_{m, n}:=\Sigma\left(S_{m, n}\right)$ were discussed by Fendley, Schoutens, and van Eerten [4] in the context of the "hard square model" in statistical mechanics. This model deals with configurations of non-overlapping ("hard") squares in $\mathbb{R}^{2}$ such that the four corners of any square in the configuration coincide with the four neighbors $(x, y \pm 1)$ and $(x \pm 1, y)$ of a lattice point $(x, y) \in \mathbb{Z}^{2}$. Identifying each such square with its center $(x, y)$, one obtains a bijection between members of the complex $\Sigma_{m, n}$ and hard square configurations that are invariant under the translation maps $(x, y) \mapsto(x+m, y)$ and $(x, y) \mapsto(x, y+n)$. See Figure 1 for an example.

\subsection{The conjectures of Fendley et al.}

Let $\Delta$ be a family of subsets of a finite set. Borrowing terminology from statistical mechanics, we define the partition function $Z(\Delta ; z)$ of $\Delta$ as

$$
Z(\Delta ; z):=\sum_{\sigma \in \Delta} z^{|\sigma|}
$$

Observe that the coefficient of $z^{k}$ in $Z(\Delta ; z)$ is the number of sets in $\Delta$ of size $k$. In particular, if $\Delta$ is a simplicial complex, then $-Z(\Delta ;-1)$ coincides with the reduced Euler characteristic of $\Delta$. Write $Z(\Delta):=Z(\Delta ;-1)$.

Conjecture 1 (Fendley et al. [4]). If $\operatorname{gcd}(m, n)=1$, then $Z\left(\Sigma_{m, n}\right)=1$.

Note that the conjecture is equivalent to saying that the unreduced Euler characteristic $\chi\left(\Sigma_{m, n}\right):=-Z\left(\Sigma_{m, n}\right)+1$ vanishes. One of the main results of this paper is a proof of Conjecture 1; see Theorems 1.1 and 2.7 below. 
A second conjecture due to Fendley et al. relates to the transfer matrix $T_{m}(z)$ of the hard square model for any fixed $m \geq 1$. The rows and columns of $T_{m}(z)$ are indexed by all subsets of $\mathbb{Z}_{m}$ avoiding pairs $(i, j)$ satisfying $j-i \equiv \pm 1(\bmod m)$. Equivalently, these subsets form the independence complex of the cycle graph $C_{m}$ with vertex set $\mathbb{Z}_{m}$ and edge set $\left\{\{i, i+1\}: i \in \mathbb{Z}_{m}\right\}$. The element on position $(\sigma, \tau)$ in $T_{m}(z)$ is defined to be

$$
t_{\sigma, \tau}(z):= \begin{cases}z^{|\sigma|} & \text { if } \sigma \cap \tau=\emptyset \\ 0 & \text { if } \sigma \cap \tau \neq \emptyset\end{cases}
$$

It is a straightforward exercise to prove that

$$
\operatorname{Tr}\left(\left(T_{m}(z)\right)^{n}\right)=Z\left(\Sigma_{m, n} ; z\right) .
$$

In particular, $\operatorname{Tr}\left(\left(T_{m}(-1)\right)^{n}\right)=Z\left(\Sigma_{m, n}\right)$. Let $P_{m}(t)$ be the characteristic polynomial of $T_{m}(-1)$; hence $P_{m}(t):=\operatorname{det}\left(t I-T_{m}(-1)\right)$.

Conjecture 2 (Fendley et al. [4]). For every $m \geq 1$, all roots of $P_{m}(t)$ are roots of unity. Specifically, $P_{m}(t)$ is a product consisting of the linear polynomial $t-1$ and $a$ number of factors of the form $t^{s} \pm 1$ such that $\operatorname{gcd}(m, s) \neq 1$.

Another of the main results of this paper is a proof of Conjecture 2; see Theorem 3.4 below.

\subsection{Balanced rhombus tilings}

Our proofs of Conjectures 1 and 2 are based on a concrete formula for $Z\left(\Sigma_{m, n}\right)$ in terms of certain rhombus tilings of the plane; see Theorem 1.1 below. The kind of rhombus tiling that we are interested in has the following properties:

- The entire plane is tiled.

- The intersection of two rhombi is either empty, a common corner, or a common side.

- The four corners of each rhombus belong to $\mathbb{Z}^{2}$.

- Each rhombus has side length $\sqrt{5}$, meaning that each side is parallel to and has the same length as $(1,2),(-1,2),(2,1)$, or $(-2,1)$.

Most rhombus tilings in the literature are built from rhombi with completely different measures; the acute angle in the rhombi is typically 60 degrees (in the case of hexagon tilings) or 36 or 72 degrees (in the case of Penrose tilings). For more information and further references, see Fulmek and Krattenthaler $[6,7]$ in the former case and Penrose [10] and de Bruijn [2] in the latter case. As far as we know, our tilings have very little, if anything, in common with these tilings.

One easily checks that a rhombus tiling with properties as above is uniquely determined by the set of rhombus corners in the tiling. From now on, we always identify a rhombus tiling with this set. 


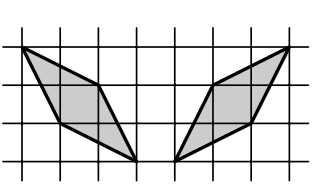

3 -rhombi

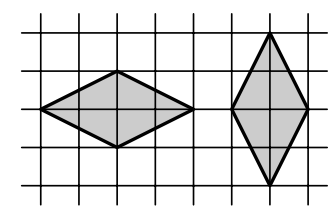

4-rhombi

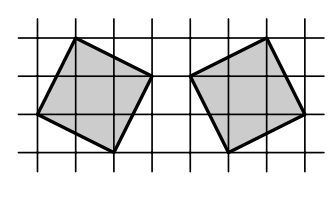

5-rhombi

Figure 2: Six different rhombi.

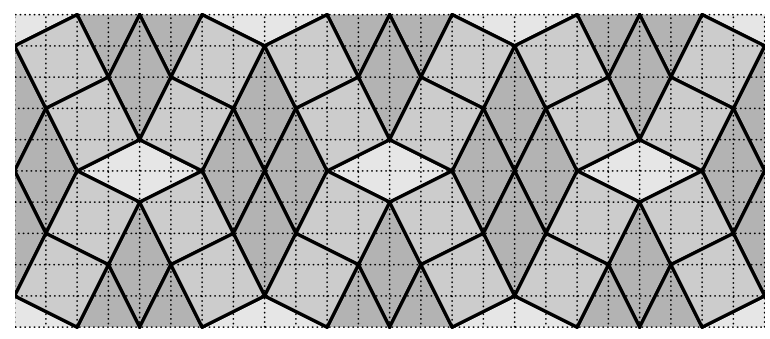

Figure 3: Portion of a balanced rhombus tiling.

We refer to a rhombus of area $k$ as a $k$-rhombus. There are six kinds of rhombi in which all corners are integer points and all sides have length $\sqrt{5}$ : two 3-rhombi, two 4-rhombi, and two 5-rhombi; see Figure 2. Note that the 5-rhombi are squares. We will restrict our attention to tilings of the plane with 4 - and 5-rhombi (i.e., the four rightmost rhombi in Figure 2); see Figure 3 for an example. Such rhombi have the property that if we divide them into four pieces via a horizontal and a vertical cut through the center, then the four resulting pieces all have the same size and shape (up to rotation and reflection). For this reason, we refer to tilings with only 4 - and 5-rhombi as balanced. Further rationale for this terminology is given in Proposition 2.1.

\subsection{Relating $\Sigma_{m, n}$ to balanced rhombus tilings}

A rhombus tiling $\rho$ is $(m, n)$-invariant if $\rho$ is invariant under translation with the vectors $(m, 0)$ and $(0, n)$. Let $R_{m, n}$ be the family of balanced $(m, n)$-invariant rhombus tilings. Each $(m, n)$-invariant rhombus tiling is a union of cosets of $\mathbb{L}_{m, n}$; recall that we identify a given tiling with its set of corners. We define $R_{m, n}^{+}$as the subfamily of $R_{m, n}$ consisting of all rhombus tilings with an even number of cosets of $\mathbb{L}_{m, n}$. Write $R_{m, n}^{-}:=R_{m, n} \backslash R_{m, n}^{+}$. For $d \in \mathbb{Z}$, define

$$
\theta_{d}:= \begin{cases}2 & \text { if } 3 \mid d \\ -1 & \text { otherwise }\end{cases}
$$

The following theorem provides a concrete formula for $Z\left(\Sigma_{m, n}\right)$ in terms of balanced rhombus tilings. 
Theorem 1.1. For every $m, n \geq 1$, we have that

$$
Z\left(\Sigma_{m, n}\right)=-(-1)^{d} \theta_{d}^{2}+\left|R_{m, n}^{+}\right|-\left|R_{m, n}^{-}\right|,
$$

where $d=\operatorname{gcd}(m, n)$.

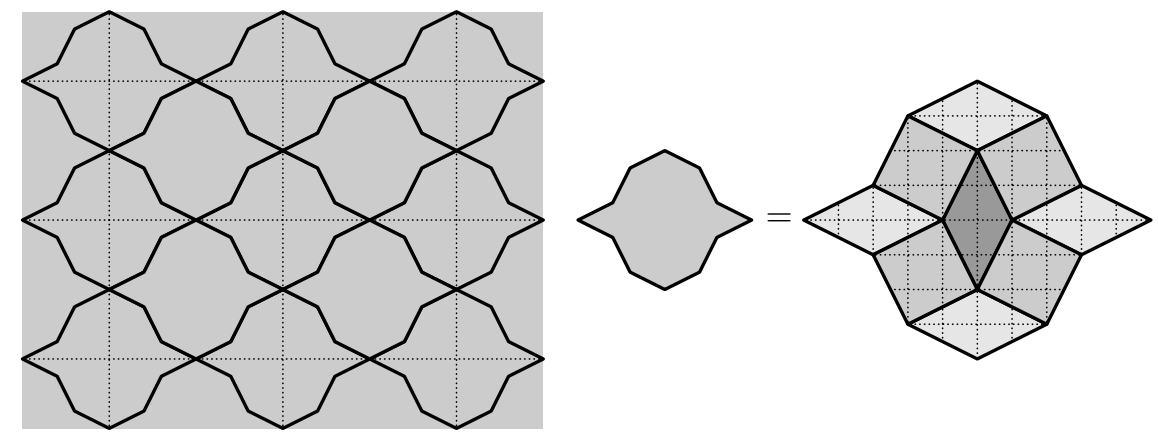

Figure 4: Translating the above rhombus tiling in all possible ways, we obtain 40 balanced $(8,10)$-invariant rhombus tilings. We get another four tilings with the same property by tiling the plane with the diamond rhombus, i.e., the dark rhombus in the very middle of the magnified picture on the right.

For example, $Z\left(\Sigma_{8,10}\right)=43$, as there are 44 tilings in $R_{8,10}$, each being the union of an even number of cosets of $\mathbb{L}_{8,10}$; see Figure 4 .

Remark. We express the value $-(-1)^{d} \theta_{d}^{2}$ in the way we do for alignment with Theorem 1.2 below.

See Section 5 for a proof of Theorem 1.1. In Section 3, we settle Conjecture 1 by proving that $R_{m, n}$ is empty whenever $\operatorname{gcd}(m, n)=1$; see Theorem 2.7. Analyzing $R_{m, n}^{+}$and $R_{m, n}^{-}$ in greater detail, showing that they satisfy certain nice enumerative properties, we also settle Conjecture 2; see Theorem 3.4. What we obtain is a formula for the characteristic polynomial $P_{m}(t)$ in terms of rhombus tilings.

\subsection{A generalization}

When proving Theorem 1.1, we will consider a slightly more general situation. Let $S$ be the infinite two-dimensional square grid; $S$ is the infinite graph on the vertex set $\mathbb{Z}^{2}$ in which there is an edge between $\left(a_{1}, a_{2}\right)$ and $\left(b_{1}, b_{2}\right)$ if and only if $\left|a_{1}-b_{1}\right|+\left|a_{2}-b_{2}\right|=1$.

Throughout this paper, $u:=\left(u_{1}, u_{2}\right)$ and $v:=\left(v_{1}, v_{2}\right)$ are two linearly independent vectors in $\mathbb{Z}^{2}$. The canonical special case is $u=(m, 0)$ and $v=(0, n)$. Let $\langle u, v\rangle$ be the subgroup of $\mathbb{Z}^{2}$ generated by $u$ and $v$. We consider the finite graph $S_{u, v}$ on the vertex set $V_{u, v}:=\mathbb{Z}^{2} /\langle u, v\rangle$ induced by the canonical map $\varphi_{u, v}: \mathbb{Z}^{2} \rightarrow V_{u, v}$; two vertices $w_{1}$ and $w_{2}$ are adjacent in $S_{u, v}$ if and only if there are adjacent vertices $w_{1}^{\prime}$ and $w_{2}^{\prime}$ in $S$ such that 
$\varphi_{u, v}\left(w_{1}^{\prime}\right)=w_{1}$ and $\varphi_{u, v}\left(w_{2}^{\prime}\right)=w_{2}$. Note that the size of $V_{u, v}$ equals the absolute value $\left|u_{1} v_{2}-u_{2} v_{1}\right|$ of the determinant of the matrix with columns $u$ and $v$. Moreover, observe that $S_{u, v}=S_{m, n}$ when $u=(m, 0)$ and $v=(0, n)$.

We refer to a rhombus tiling as $\langle u, v\rangle$-invariant if the tiling is invariant under the translation $x \mapsto x+w$ for every $w \in\langle u, v\rangle$. Of course, this is equivalent to the tiling being invariant under the two translations $x \mapsto x+u$ and $x \mapsto x+v$. Define $R_{u, v}$ to be the family of balanced $\langle u, v\rangle$-invariant rhombus tilings. Let $R_{u, v}^{+}$be the subfamily of $R_{u, v}$ consisting of those rhombus tilings with an even number of cosets of $\langle u, v\rangle$ and write $R_{u, v}^{-}:=R_{u, v} \backslash R_{u, v}^{+}$.

Write $\Sigma_{u, v}:=\Sigma\left(S_{u, v}\right)$. Our generalization of Theorem 1.1 reads as follows:

Theorem 1.2. Write $d:=\operatorname{gcd}\left(u_{1}-u_{2}, v_{1}-v_{2}\right)$ and $d^{*}:=\operatorname{gcd}\left(u_{1}+u_{2}, v_{1}+v_{2}\right)$. Then

$$
Z\left(\Sigma_{u, v}\right)=-(-1)^{d} \theta_{d} \theta_{d^{*}}+\left|R_{u, v}^{+}\right|-\left|R_{u, v}^{-}\right|,
$$

where $\theta_{d}$ is defined as in (1) in Section 1.3.

Since $d=d^{*}$ if $u=(m, 0)$ and $v=(0, n)$, Theorem 1.2 implies Theorem 1.1. Note that the expression $(-1)^{d} \theta_{d} \theta_{d^{*}}$ is symmetric in $d$ and $d^{*} ; d$ is even if and only if $d^{*}$ is even.

Our analysis of the partition function of $\Sigma_{u, v}$ does not seem to provide much insight into the homology of the complex. Nevertheless, it turns out [8] that one may exploit the nice structure of balanced rhombus tilings to detect nonvanishing free homology in dimension $k-1$ whenever there are balanced $\langle u, v\rangle$-invariant rhombus tilings with exactly $k$ cosets of $\langle u, v\rangle$.

\section{Organization of the paper}

We deal with periodic and balanced rhombus tilings in Section 2, proving that such tilings satisfy certain nice properties. Section 3 is devoted to settling Conjectures 1 and 2, assuming that Theorem 1.2 is true; we postpone the difficult proof of Theorem 1.2 until Section 5. Translation permutations form an important part of this proof; we discuss such permutations in Section 4. Finally, we make some concluding remarks in Section 6.

\section{Periodic and balanced rhombus tilings}

For any element $x \in \mathbb{Z}^{2}$, define $\mathrm{s}(x):=x+(1,0)$ (south), $\mathrm{e}(x):=x+(0,1)$ (east), $\mathrm{n}(x):=x+(-1,0)$ (north), and $\mathrm{w}(x):=x+(0,-1)$ (west); recall our matrix convention for indexing elements in $\mathbb{Z}^{2}$. Given a set $\sigma$, we refer to an element $x$ as blocked in $\sigma$ if at least one of its neighbors $\mathrm{s}(x), \mathrm{e}(x), \mathrm{n}(x)$, and $\mathrm{w}(x)$ belongs to $\sigma$. Such a neighbor is said to block $x$.

Proposition 2.1. A nonempty set $\rho \subset \mathbb{Z}^{2}$ is a balanced rhombus tiling if and only if all elements in $\rho$ are pairwise non-blocking and the following holds: For each $x \in \rho$ and each choice of signs $t, u \in\{+1,-1\}$, exactly one of the elements $\mathbf{s}^{t} \mathrm{e}^{2 u}(x)$ and $\mathbf{s}^{2 t} \mathrm{e}^{u}(x)$ belongs to $\rho$. 
Proof. ( $\Longrightarrow)$ Suppose that $\rho$ is a balanced rhombus tiling. By symmetry, it suffices to consider the case $t=u=1$. If both $y:=\operatorname{se}^{2}(x)$ and $z:=\mathrm{s}^{2} \mathrm{e}(x)$ belong to $\rho$, then the rhombus defined by the three corners $x, y, z$ has area three and is hence not allowed. If neither $y$ nor $z$ belongs to $\rho$, then we have another contradiction, as the region just to the south-east of $p$ cannot be a 4 - or 5 -rhombus; consider Figure 2.

$(\Longleftarrow)$ Suppose that $\rho$ satisfies the latter condition in the lemma. Partition $\mathbb{Z}^{2}$ into regions by drawing a line segment between any two elements in $\rho$ on distance $\sqrt{5}$. By symmetry, it suffices to prove that the region just to the east of any element $x$ in $\rho$ is a 4- or 5-rhombus. We have four cases:

- $y:=\operatorname{se}^{2}(x)$ and $z:=\mathrm{ne}^{2}(x)$ belong to $\sigma$. Since $\mathrm{n}^{2} \mathrm{e}(y)=\mathrm{e}(z)$ is blocked by $z$, we have that $\mathrm{ne}^{2}(y)=\mathrm{e}^{4}(x)$ belongs to $\sigma$, which yields a 4 -rhombus.

- $y:=\operatorname{se}^{2}(x)$ and $z:=\mathrm{n}^{2} \mathrm{e}(x)$ belong to $\sigma$. Since $\mathrm{s}^{2} \mathrm{e}(z)=\mathrm{n}(y)$ is blocked by $y$, we have that $\operatorname{se}^{2}(z)=\mathrm{ne}^{3}(x)$ belongs to $\sigma$, which yields a 5-rhombus.

- $\mathrm{s}^{2} \mathrm{e}(x)$ and $\mathrm{ne}^{2}(x)$ belong to $\sigma$. By symmetry, this case is analogous to the second case.

- $y:=\mathrm{s}^{2} \mathrm{e}(x)$ and $z:=\mathrm{n}^{2} \mathrm{e}(x)$ belong to $\sigma$. If $\mathrm{e}^{2}(x) \notin \sigma$, then $y^{\prime}:=\mathrm{se}^{2}(z) \in \sigma$. However, $\mathrm{sw}^{2}\left(y^{\prime}\right)=\mathrm{e}(x)$ is blocked by $x$ and hence not in $\sigma$. Moreover, $\mathrm{s}^{2} \mathrm{w}\left(y^{\prime}\right)=$ $\mathrm{se}^{2}(x)$ is not in $\sigma$ either, because $\mathrm{s}^{2} \mathrm{e}(x) \in \sigma$. This is a contradiction; hence $\mathrm{e}^{2}(x)$ belongs to $\sigma$, which yields a 4 -rhombus.

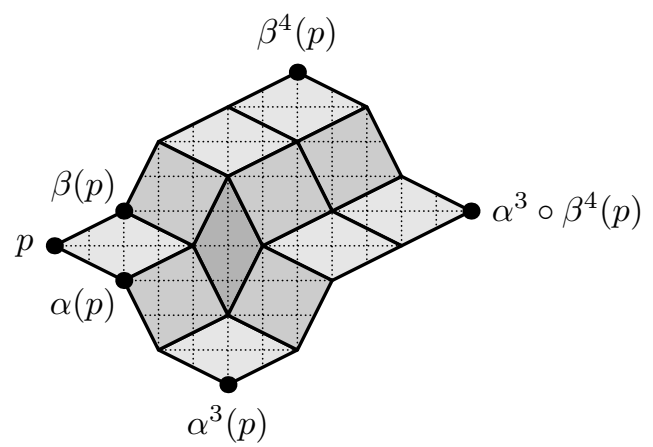

Figure 5: Illustration of the functions $\alpha$ and $\beta$. As predicted by Lemma 2.2, we have that $\alpha^{3} \circ \beta^{4}(p)=\alpha^{3}(p)+\beta^{4}(p)-p$.

Let $\rho$ be a balanced rhombus tiling. For a given element $p \in \rho$, let $\alpha(p)$ be the one element among $\mathrm{s}^{2} \mathrm{e}(p)$ and $\mathrm{se}^{2}(p)$ that belongs to $\rho$; by Proposition 2.1, $\alpha(p)$ is welldefined. Furthermore, let $\beta(p)$ be the one element among $\mathrm{n}^{2} \mathrm{e}(p)$ and $\mathrm{ne}^{2}(p)$ that belongs to $\rho$. See Figure 5 for an illustration. By symmetry, $\alpha$ and $\beta$ have well-defined inverses; hence $\alpha^{r}(p)$ and $\beta^{r}(p)$ are well-defined for all $r \in \mathbb{Z}$. 
Lemma 2.2. For any balanced rhombus tiling $\rho$, the functions $\alpha$ and $\beta$ satisfy the identity

$$
\alpha^{r} \circ \beta^{s}(p)=\beta^{s} \circ \alpha^{r}(p)=\alpha^{r}(p)+\beta^{s}(p)-p
$$

for all $p \in \rho$ and $r, s \in \mathbb{Z}$.

Proof. The lemma is trivially true for $r=0$ or $s=0$. By symmetry, it suffices to consider the case $r, s \geq 1$. Use induction on $r, s$. For $r=s=1$, we have that $p, \alpha(p), \beta(p)$ constitute three of the corners in a rhombus contained in the tiling. The fourth corner is clearly $\alpha(p)+\beta(p)-p$, which is equal to $\beta(\alpha(p))$ and $\alpha(\beta(p))$ as desired. This also yields that $\alpha$ and $\beta$ commmute.

Now, suppose that either $r$ or $s$, say $s$, is at least two. Assuming inductively that the lemma holds for smaller values of $s$, we obtain that

$$
\begin{aligned}
\alpha^{r} \circ \beta^{s}(p) & =\alpha^{r} \circ \beta^{s-1}(\beta(p))=\alpha^{r} \circ \beta(p)+\beta^{s-1} \circ \beta(p)-\beta(p) \\
& =\alpha^{r}(p)+\beta(p)-p+\beta^{s}(p)-\beta(p)=\alpha^{r}(p)+\beta^{s}(p)-p .
\end{aligned}
$$

The following lemma is straightforward to prove.

Lemma 2.3. Let $\rho$ be a balanced rhombus tiling and let $p, q \in \rho$. Then there are unique integers $r$ and $s$ such that $q=\alpha^{r} \circ \beta^{s}(p)$.

For $i \in \mathbb{Z}$, define $\delta_{i}(p):=\alpha^{i}(p)-\alpha^{i-1}(p)$ and $\epsilon_{i}(p):=\beta^{i}(p)-\beta^{i-1}(p)$; by symmetry, this is well-defined for $i \leq 0$.

Corollary 2.4. Let $\rho$ be a balanced rhombus tiling, let $p \in \rho$, and let $q:=\alpha^{r} \circ \beta^{s}(p)$ be another element in $\rho$. Then $\delta_{i}(q)=\delta_{i+r}(p)$ and $\epsilon_{i}(q)=\epsilon_{i+s}(p)$.

Proof. By Lemma 2.2, we have that

$$
\begin{aligned}
\delta_{i}(q) & =\alpha^{i}(q)-\alpha^{i-1}(q)=\alpha^{r+i} \circ \beta^{s}(p)-\alpha^{r+i-1} \circ \beta^{s}(p) \\
& =\alpha^{r+i}(p)+\beta^{s}(p)-p-\left(\alpha^{r+i-1}(p)+\beta^{s}(p)-p\right) \\
& =\alpha^{r+i}(p)-\alpha^{r+i-1}(p)=\delta_{i+r}(p) .
\end{aligned}
$$

The proof for $\epsilon_{i}(q)$ is analogous.

Recall that $u$ and $v$ are linearly independent integer vectors. Let $\rho$ be a balanced $\langle u, v\rangle$-invariant rhombus tiling. By finiteness of $\mathbb{Z}^{2} /\langle u, v\rangle$ and Lemma 2.2, the sequences $\left(\delta_{i}(p): i \in \mathbb{Z}\right)$ and $\left(\epsilon_{i}(p): i \in \mathbb{Z}\right)$ are periodic. Let $K$ and $L$ be minimal such that $\delta_{i}=\delta_{i+K}(p)$ and $\epsilon_{i}(p)=\epsilon_{i+L}(p)$ for all $i \in \mathbb{Z}$. By Corollary 2.4, $K$ and $L$ do not depend on $p$. Define

$$
\begin{aligned}
& x(p):=\alpha^{K}(p)-p=\sum_{i=1}^{K} \delta_{i}(p) \\
& y(p):=\beta^{L}(p)-p=\sum_{i=1}^{L} \epsilon_{i}(p) .
\end{aligned}
$$




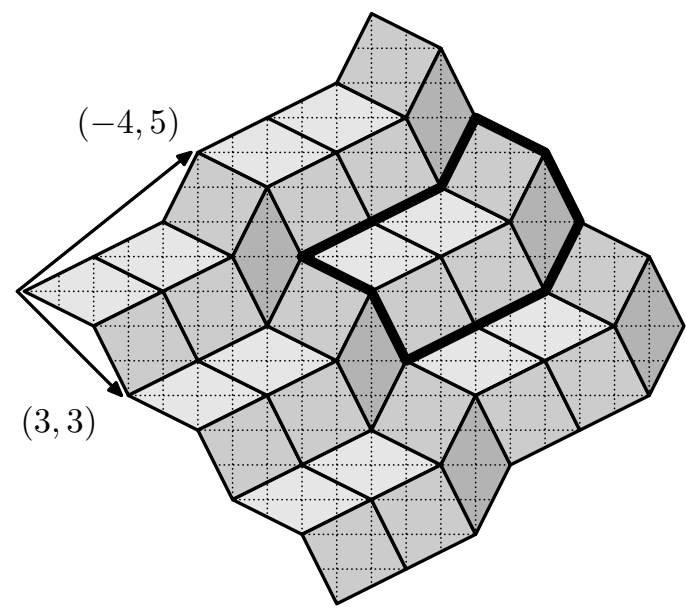

Figure 6: Portion of a balanced periodic rhombus tiling with axes $(3,3)$ and $(4,-5)$. The border of one "period" of the tiling is marked in bold.

$x(p)$ and $y(p)$ are the axes of $\rho$. See Figure 6 for an illustration. We claim that the axes do not depend on the choice of origin $p$. Namely, if $q:=\alpha^{r} \circ \beta^{s}(p)$, then Corollary 2.4 yields that

$$
x(q)=\alpha^{K}(q)-q=\sum_{i=1}^{K} \delta_{i}(q)=\sum_{i=r+1}^{K+r} \delta_{i}(p)=\sum_{i=1}^{K} \delta_{i}(p)=x(p) .
$$

By symmetry, the same property holds for $y(p)$. We summarize:

Lemma 2.5. Let $\rho$ be a balanced $\langle u, v\rangle$-invariant rhombus tiling. Then, for every $p, q \in$ $V(\rho), x(p)=x(q)$ and $y(p)=y(q)$.

Theorem 2.6. Let $x:=\left(x_{1}, x_{2}\right)$ and $y:=\left(-y_{1}, y_{2}\right)$ be vectors such that $x_{1}, x_{2}, y_{1}$, and $y_{2}$ are all positive. Then there are balanced $\langle u, v\rangle$-invariant rhombus tilings with axes $x^{\prime}$ and $y^{\prime}$ such that $x$ and $y$ are integer multiples of $x^{\prime}$ and $y^{\prime}$, respectively, if and only if the following hold:

(i) $x_{1} / 2 \leq x_{2} \leq 2 x_{1}$ and $y_{1} / 2 \leq y_{2} \leq 2 y_{1}$.

(ii) $x_{1}+x_{2}$ and $y_{1}+y_{2}$ are divisible by three.

(iii) $\langle x, y\rangle$ contains $\langle u, v\rangle$.

Assuming that the above conditions hold and defining

$$
\left(\begin{array}{ll}
a & b \\
c & d
\end{array}\right):=\frac{1}{3} \cdot\left(\begin{array}{ll}
x_{1} & x_{2} \\
y_{1} & y_{2}
\end{array}\right) \cdot\left(\begin{array}{cc}
-1 & 2 \\
2 & -1
\end{array}\right)
$$


(i.e., $x=a \cdot(1,2)+b \cdot(2,1)$ and $y=c \cdot(-1,2)+d \cdot(-2,1))$, the number of such tilings is

$$
\left(\begin{array}{c}
a+b \\
a
\end{array}\right)\left(\begin{array}{c}
c+d \\
c
\end{array}\right) \cdot\left(\frac{a d+b c}{(a+b)(c+d)}+4\right) .
$$

Proof. It is clear that the axes of any $\langle u, v\rangle$-invariant rhombus tiling must satisfy conditions (i)-(iii); $x^{\prime}$ is a nonnegative sum of vectors of the form $(1,2)$ and $(2,1)$, whereas $y^{\prime}$ is a nonnegative sum of vectors of the form $(-1,2)$ and $(-2,1)$.

Conversely, suppose that conditions (i)-(iii) are satisfied and define $a, b, c, d$ as in the theorem. Conditions (i)-(ii) mean that these numbers are all nonnegative integers such that $a+b$ and $c+d$ are positive. Write $K:=a+b$ and $L:=c+d$. Let $\left(\lambda_{1}, \ldots, \lambda_{K}\right)$ and $\left(\mu_{1}, \ldots, \mu_{L}\right)$ be binary sequences such that $\sum_{i} \lambda_{i}=a$ and $\sum_{i} \mu_{i}=c$. Define $\lambda_{i}$ and $\mu_{i}$ for all $i \in \mathbb{Z}$ via the identities $\lambda_{i}=\lambda_{i+K}$ and $\mu_{i}=\mu_{i+L}$. Define $\delta_{i}$ and $\epsilon_{i}$ as

$$
\delta_{i}:= \begin{cases}(1,2) & \text { if } \lambda_{i}=1 \\ (2,1) & \text { if } \lambda_{i}=0\end{cases}
$$

and

$$
\epsilon_{i}:= \begin{cases}(-1,2) & \text { if } \mu_{i}=1 \\ (-2,1) & \text { if } \mu_{i}=0\end{cases}
$$

Define the set $\left\{p_{i, j}: i, j \in \mathbb{Z}\right\}$ by $p_{0,0}=(0,0)$ and

$$
\left\{\begin{array}{l}
p_{r, s}-p_{r-1, s}=\delta_{r} \\
p_{r, s}-p_{r, s-1}=\epsilon_{s}
\end{array}\right.
$$

for all $r, s \in \mathbb{Z}$. This means that $p_{r, s}=\sum_{i=1}^{r} \delta_{i}+\sum_{j=1}^{s} \epsilon_{j}$ for $r, s \geq 0$. One easily checks that $\left\{p_{r-1, s-1}, p_{r-1, s}, p_{r, s-1}, p_{r, s}\right\}$ is the set of corners in a 4 - or 5 -rhombus for each $r, s \in \mathbb{Z}$.

To prove that $\left\{p_{r, s}: r, s \in \mathbb{Z}\right\}$ is a rhombus tiling, it suffices by Proposition 2.1 to show that $p_{r, s}$ is not equal or adjacent to $p_{r^{\prime}, s^{\prime}}$ unless $(r, s)=\left(r^{\prime}, s^{\prime}\right)$. This is a straightforward exercise.

Since $\sum_{i=1}^{K} \delta_{i}=a \cdot(1,2)+(K-a) \cdot(2,1)=x$ and $\sum_{i=1}^{L} \epsilon_{i}=c \cdot(-1,2)+(L-c) \cdot(-2,1)=$ $y$, it follows that $x$ and $y$ are integer multiples of the axes of the tiling. This settles the other direction in the first part of the proof.

To compute the number of rhombus tilings with desired properties, note that the previous results of this section imply that these tilings have properties as described in this proof, except that we do not necessarily have that $(0,0)$ is a corner.

First, let us compute the number of tilings having $(0,0)$ as a corner such that the rhombus $P$ in which $(0,0)$ is the western corner has a given fixed area. The number of sequences $\left\{\delta_{i}\right\}$ such that $\delta_{1}=(1,2)$ is $\left(\begin{array}{c}a+b-1 \\ a-1\end{array}\right)$; the number of sequences such that $\delta_{1}=(2,1)$ is $\left(\begin{array}{c}a+b-1 \\ a\end{array}\right)$. The number of sequences $\left\{\epsilon_{i}\right\}$ such that $\epsilon_{1}=(-1,2)$ is $\left(\begin{array}{c}c+d-1 \\ c-1\end{array}\right)$; the number of sequences such that $\epsilon_{1}=(-2,1)$ is $\left(\begin{array}{c}c+d-1 \\ c\end{array}\right)$. The area of $P$ being five means that the second coefficients of $\delta_{1}$ and $\epsilon_{1}$ do not coincide. Hence there are

$$
\left(\begin{array}{c}
a+b-1 \\
a-1
\end{array}\right)\left(\begin{array}{c}
c+d-1 \\
c
\end{array}\right)+\left(\begin{array}{c}
a+b-1 \\
a
\end{array}\right)\left(\begin{array}{c}
c+d-1 \\
c-1
\end{array}\right)
$$


tilings such that the area of $P$ is five and there are

$$
\left(\begin{array}{c}
a+b-1 \\
a-1
\end{array}\right)\left(\begin{array}{c}
c+d-1 \\
c-1
\end{array}\right)+\left(\begin{array}{c}
a+b-1 \\
a
\end{array}\right)\left(\begin{array}{c}
c+d-1 \\
c
\end{array}\right)
$$

tilings such that the area of $P$ is four.

Now, let us compute the total number of tilings. Fixing as starting point $p_{0,0}$ the western corner of the rhombus in which $(0,0)$ is either the western corner or an interior point, we obtain that the total number of tilings that we want to compute equals

$$
5\left(\left(\begin{array}{c}
a+b-1 \\
a-1
\end{array}\right)\left(\begin{array}{c}
c+d-1 \\
c
\end{array}\right)+\left(\begin{array}{c}
a+b-1 \\
a
\end{array}\right)\left(\begin{array}{c}
c+d-1 \\
c-1
\end{array}\right)\right)+4\left(\left(\begin{array}{c}
a+b-1 \\
a-1
\end{array}\right)\left(\begin{array}{c}
c+d-1 \\
c-1
\end{array}\right)+\left(\begin{array}{c}
a+b-1 \\
a
\end{array}\right)\left(\begin{array}{c}
c+d-1 \\
c
\end{array}\right)\right) .
$$

This is easily seen to equal the expression in the theorem; hence we are done.

Theorem 2.7. If $m$ and $n$ are coprime, then there are no balanced $(m, n)$-invariant rhombus tilings.

Proof. Suppose that there is such a tiling $\rho$ and let $x:=\left(x_{1}, x_{2}\right)$ and $y:=\left(-y_{1}, y_{2}\right)$ be the axes of $\rho ; x_{1}, x_{2}, y_{1}, y_{2}$ are all positive integers. Since $\langle x, y\rangle$ contains $\mathbb{L}_{m, n}$ by Theorem 2.6, we have that there is a $2 \times 2$ integer matrix $A$ such that

$$
\left(\begin{array}{cc}
x_{1} & -y_{1} \\
x_{2} & y_{2}
\end{array}\right) \cdot A=\left(\begin{array}{cc}
m & 0 \\
0 & n
\end{array}\right)
$$

However, we also have that the determinant $x_{1} y_{2}+x_{2} y_{1}$ divides $m n$. Define $m_{0}$ and $n_{0}$ such that $x_{1} y_{2}+x_{2} y_{1}=m_{0} n_{0}$, where $m_{0} \mid m$ and $n_{0} \mid n$. Since

$$
A=\frac{1}{m_{0} n_{0}}\left(\begin{array}{cc}
m y_{2} & n y_{1} \\
-m x_{2} & n x_{1}
\end{array}\right)
$$

it follows that $m_{0}$ divides $x_{1}$ and $y_{1}$ and that $n_{0}$ divides $x_{2}$ and $y_{2}$. As a consequence,

$$
\left(\begin{array}{cc}
x_{1} / m_{0} & -y_{1} / m_{0} \\
x_{2} / n_{0} & y_{2} / n_{0}
\end{array}\right)
$$

is an integer matrix with unit determinant, which is impossible.

\section{Establishing Conjectures 1 and 2}

For continuity, we show how to deduce Conjectures 1 and 2 from Theorem 1.2 before actually proving the theorem, which we do in Section 5. Nothing in the present section is used in Sections 4 and 5 .

First, let us settle Conjecture 1:

Corollary 3.1. If $m$ and $n$ are coprime, then $Z\left(\Sigma_{m, n}\right)=1$.

Proof. This is an immediate consequence of Theorems 1.2 and 2.7. 
For $m \geq 1$, let $R_{m}$ be the family of balanced rhombus tilings that are $(m, n)$-invariant for some $n \geq 1 .^{1}$ For two vectors $x$ and $y$ to satisfy the conditions in Theorem 2.6, we must have that $x_{1}+y_{1} \leq m$. In particular, there are only finitely many vectors $x$ and $y$ with desired properties, which implies that $R_{m}$ is a finite family.

Theorem 3.2. Let $f(t):=\sum_{m \geq 1}\left|R_{m}\right| t^{m}$. Then

$$
f(t)=\frac{t+2 t^{2}}{1-t-t^{2}}+\frac{-t+t^{2}-4 t^{3}}{1+t^{3}} .
$$

Equivalently,

$$
\left|R_{m}\right|=\left(\frac{1+\sqrt{5}}{2}\right)^{m}+\left(\frac{1-\sqrt{5}}{2}\right)^{m}+(-1)^{m} \theta_{m}^{2},
$$

where $\theta_{m}$ is defined as in (1) in Section 1.3.

Proof. First, we compute the number $b_{m}$ of balanced rhombus tilings that are $(m, n)$ invariant for some $n$ and contain the origin $p_{0}:=(0,0)$. Let $\rho$ be such a tiling. $(m, n)$ invariance implies that $\rho$ contains the element $p_{m}:=(m, 0)$.

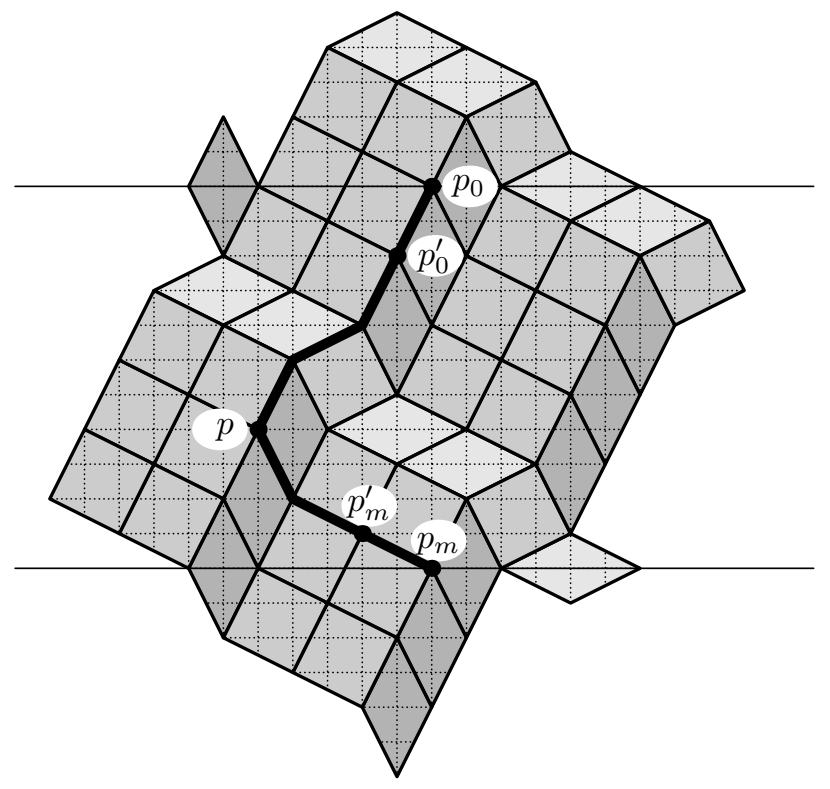

Figure 7: Portion of a rhombus tiling invariant under translation with the vector $(m, 0)$ for $m=11$, along with the elements $p_{0}, p_{m}, p, p_{0}^{\prime}, p_{m}^{\prime}$ in the proof of Theorem 3.2. Note that $p_{m}^{\prime}=p_{0}^{\prime}+(m-3,-1)=(8,-1)$.

Let $\alpha$ and $\beta$ be defined as in Section 2 (recall Figure 5). Lemma 2.3 yields unique elements $r$ and $s$ such that $\alpha^{r} \beta^{-s}\left(p_{0}\right)=p_{m}$. It is clear that $r$ and $s$ are both positive. Define $p:=\beta^{-s}\left(p_{0}\right)=\alpha^{-r}\left(p_{m}\right)$. See Figure 7 for an illustration.

\footnotetext{
${ }^{1}$ It is not hard to show that $R_{m}$ coincides with the family of balanced rhombus tilings that are invariant under translation with the vector $(m, 0)$.
} 
We claim that $p_{m}-p$ and $p_{0}-p$ are multiples of the two axes of the tiling $\rho$. Namely, let $t$ be an integer. By Lemma 2.2, we have that

$$
\begin{aligned}
\alpha^{t}(p) & =\alpha^{t-r}\left(p_{m}\right)=\alpha^{t-r}\left(p_{0}\right)+p_{m}-p_{0} \\
& =\alpha^{t-r}\left(\beta^{s}(p)\right)+p_{m}-p_{0}=\alpha^{t-r}(p)+\beta^{s}(p)-p+p_{m}-p_{0} .
\end{aligned}
$$

As a consequence,

$$
\delta_{t}(p):=\alpha^{t}(p)-\alpha^{t-1}(p)=\alpha^{t-r}(p)-\alpha^{t-r-1}(p)=\delta_{t-r}(p),
$$

which implies that the period of $\left(\delta_{i}(p): i \in \mathbb{Z}\right)$ divides $r$. Analogously, we obtain that the period of $\left(\epsilon_{i}(p):=\beta^{i}(p)-\beta^{i-1}(p): i \in \mathbb{Z}\right)$ divides $s$. The claim follows.

To summarize, there is one rhombus tiling to count for each point $p$ and each pair of paths $\left(\mathrm{P}_{0}, \mathrm{P}_{m}\right)$ from $p_{0}$ and $p_{m}$, respectively, to $p$ with allowed steps being $(1,-2)$ and $(2,-1)$ for $\mathrm{P}_{0}$ and $(-1,-2)$ and $(-2,-1)$ for $\mathrm{P}_{m}$. See Figure 7 for an illustration.

Going one step in each of the paths, we end up on new positions $p_{0}^{\prime}$ and $p_{m}^{\prime}$. If the first steps in $\mathrm{P}_{0}$ and $\mathrm{P}_{m}$ are $(1,-2)$ and $(-1,-2)$, respectively, then $p_{m}^{\prime}=p_{0}^{\prime}+(m-2,0)$. Hence there are $b_{m-2}$ such paths. Defining $b_{0}:=1$ and $b_{i}:=0$ if $i<0$, this is true for all $m$. If the first steps in $P_{0}$ and $P_{m}$ are $(2,-1)$ and $(-2,-1)$, respectively, then $p_{m}^{\prime}=p_{0}^{\prime}+(m-4,0)$. Hence there are $b_{m-4}$ such paths.

The remaining case is that the second coordinates of the first steps in $\mathrm{P}_{0}$ and $\mathrm{P}_{m}$ do not coincide. By symmetry, we may assume that the steps are $(1,-2)$ and $(-2,-1)$, which yields the identity $p_{m}^{\prime}=p_{0}^{\prime}+(m-3,1)$. Let $c_{m-3}$ be the number of such paths. We obtain the recursion

$$
b_{m}=b_{m-2}+b_{m-4}+2 c_{m-3} .
$$

Now, proceed with the last case and go another step on the path $\mathrm{P}_{m}$. First, suppose that the second step is $(-1,-2)$. This yields a point $p_{m}^{\prime \prime}$ satisfying $p_{m}^{\prime \prime}=p_{0}^{\prime}+(m-4,-1)$. Hence there are $c_{m-4}$ such paths. Next, suppose that the second step is $(-2,-1)$. Then we obtain $p_{m}^{\prime \prime}=p_{0}^{\prime}+(m-5,0)$. Hence there are $b_{m-5}$ such paths. Summarizing, we get

$$
c_{m-3}=c_{m-4}+b_{m-5},
$$

which implies that

$$
\begin{aligned}
b_{m}-b_{m-1} & =b_{m-2}-b_{m-3}+b_{m-4}-b_{m-5}+2\left(c_{m-3}-c_{m-4}\right) \\
& \Longleftrightarrow b_{m}-b_{m-1}-b_{m-2}+b_{m-3}-b_{m-4}-b_{m-5}=0 ;
\end{aligned}
$$

use (2). Since $b_{1}=b_{3}=c_{0}=c_{1}=0$ and $b_{0}=b_{2}=c_{2}=1$, we obtain that $b_{4}=2$ and hence that

$$
B(t):=\sum_{i \geq 0} b_{i} t^{i}=\frac{1-t}{1-t-t^{2}+t^{3}-t^{4}-t^{5}}=\frac{1-t}{\left(1-t-t^{2}\right)\left(1+t^{3}\right)} .
$$

It remains to compute the total number of tilings, not only those containing the origin. Let $p_{0}$ be the point in a given tiling $\rho$ with the property that the origin equals $p_{0}$ or is an 
inner point in the rhombus with eastern corner $p_{0}$. Using exactly the same approach as above, taking one step on each of the paths $\mathrm{P}_{0}$ and $\mathrm{P}_{m}$, we obtain that

$$
\left|R_{m}\right|=4 b_{m-2}+4 b_{m-4}+5 c_{m-3}+5 c_{m-3}=5 b_{m}-b_{m-2}-b_{m-4} ;
$$

use (2) for the second equality. To understand the first equality, note that the first term corresponds to the case that the first steps in $P_{0}$ and $P_{m}$ are $(1,-2)$ and $(-1,-2)$, respectively. This yields a 4 -rhombus just to the west of $p_{0}$, which explains the factor four. The other three terms are explained in the same manner.

Applying (3) and observing that $\left|R_{1}\right|=\left|R_{3}\right|=0$ and $\left|R_{2}\right|=4$, we conclude that

$$
f(t)=\frac{\left(5-t^{2}-t^{4}\right)(1-t)}{\left(1-t-t^{2}\right)\left(1+t^{3}\right)}-5 .
$$

A straightforward computation yields the identities in the theorem.

Proposition 3.3. Let $D_{m}$ be the size of $\Sigma\left(C_{m}\right)$, where $C_{m}$ is the cycle graph on the vertex set $\mathbb{Z}_{m}$. Equivalently, $D_{m}$ is the number of rows in the transfer matrix $T_{m}(z)$. Then

$$
D_{m}=\left(\frac{1+\sqrt{5}}{2}\right)^{m}+\left(\frac{1-\sqrt{5}}{2}\right)^{m} \text {. }
$$

Equivalently, $D_{m}=\left|R_{m}\right|-(-1)^{m} \theta_{m}^{2}$ where $\theta_{m}$ is defined as in (1) in Section 1.3.

Proof. Clearly, $D_{1}=1$ and $D_{2}=3$. For $m \geq 3$, let $\Sigma_{1}$ be the subfamily of $\Sigma\left(C_{m}\right)$ consisting of all $\sigma$ such that $m-1 \notin \sigma$ and such that at most one of $m-2$ and 0 belongs to $\sigma$. We obtain a bijection from $\Sigma_{1}$ to $\Sigma\left(C_{m-1}\right)$ by removing the vertex $m-1$. The remaining family $\Sigma_{2}$ consists of all $\sigma$ such that either of the following holds:

- $m-2,0 \in \sigma$ and $m-1 \notin \sigma$.

- $m-2,0 \notin \sigma$ and $m-1 \in \sigma$.

We obtain a bijection from $\Sigma_{2}$ to $\Sigma\left(C_{m-2}\right)$ by removing the vertices $m-2$ and $m-1$. Combining the two bijections, we obtain the identity $D_{m}=D_{m-1}+D_{m-2}$, which yields the desired formula.

Let $R_{m}^{-}$be the family of tilings $\rho$ in $R_{m}$ such that $\rho \in R_{m, n}^{-}$for some $n$. Write $R_{m}^{+}:=R_{m} \backslash R_{m}^{-}$. For a tiling $\rho$, let $\nu:=\nu(\rho)$ be minimal such that $\rho$ is invariant under translation with $(0, \nu)$. One readily verifies that $\rho \in R_{m, n}^{-}$if and only if $\rho \in R_{m}^{-}$and $n / \nu(\rho)$ is an odd integer. Equivalently, $\rho \in R_{m, n}^{+}$if and only if either $\rho \in R_{m}^{+}$and $n / \nu(\rho)$ is an integer or $\rho \in R_{m}^{-}$and $n / \nu(\rho)$ is an even integer.

For each positive integer $n$, let $\psi_{m}^{+}(n)$ be the number of tilings $\rho \in R_{m}^{+}$such that $\nu(\rho)=n$. Define $\psi_{m}^{-}(n)$ analogously for tilings in $R_{m}^{-}$. It is clear that $\psi_{m}^{+}(n)$ and $\psi_{m}^{-}(n)$ are integer multiples of $n$, because a given tiling $\rho$ such that $\nu(\rho)=n$ must have the property that the tilings $\rho, \rho+(0,1), \rho+(0,2), \ldots, \rho+(0, n-1)$ are all distinct; otherwise, $\nu(\rho)$ would be a proper divisor of $n$. 

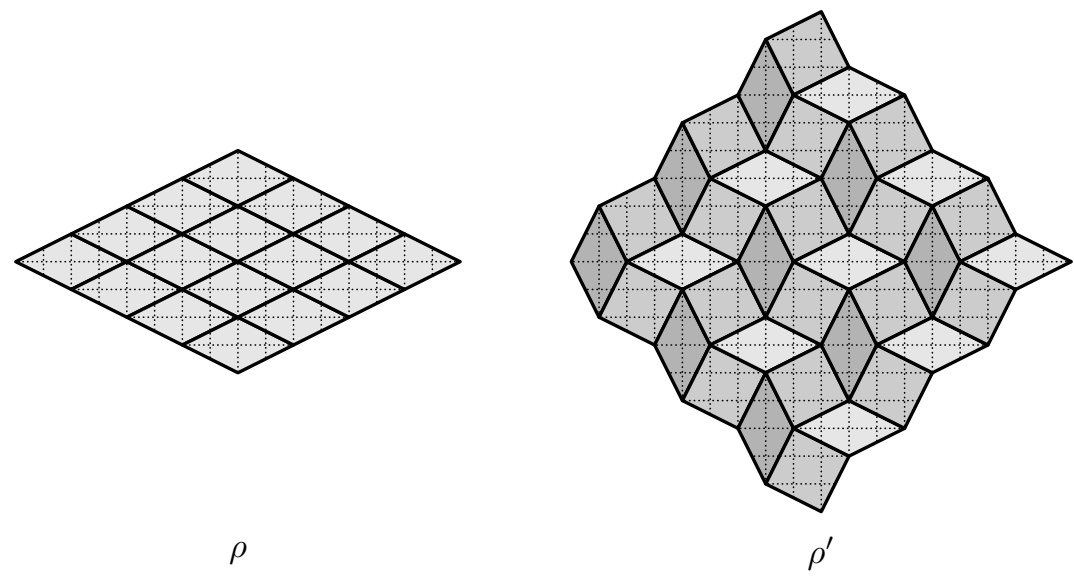

Figure 8: On the left a rhombus tiling $\rho$ invariant under four-step horizontal and two-step vertical translation. On the right a rhombus tiling $\rho^{\prime}$ invariant under six-step horizontal and vertical translation.

Example. One obtains all rhombus tilings in $R_{6}$ via translation of the two tilings in Figure 8. For the tiling $\rho$ on the left, $\nu(\rho)=4$ and $\rho \in R_{6}^{+}$, because $\nu=4$ is minimal such that $\rho+(0, \nu)=\rho$ and $\rho$ contains six cosets of $\mathbb{L}_{6,4}$, which is even. For the tiling $\rho^{\prime}$ on the right, $\nu\left(\rho^{\prime}\right)=6$ and $\rho^{\prime} \in R_{6}^{+} ; \rho^{\prime}$ contains eight cosets of $\mathbb{L}_{6,6}$, which is again even. Since there are four distinct translations of $\rho$ and 18 distinct translations of $\rho^{\prime}$, we conclude that $\psi_{6}^{ \pm}(n)$ is always zero with the two exceptions $\psi_{6}^{+}(4)=4$ and $\psi_{6}^{+}(6)=18$.

Theorem 3.4. For any integer $m \geq 1$, the characteristic polynomial $P_{m}(t)$ of the transfer matrix $T_{m}:=T_{m}(-1)$ satisfies

$$
P_{m}(t)=g_{m}(t) \cdot \prod_{n \geq 1}\left(t^{n}-1\right)^{\psi_{m}^{+}(n) / n} \cdot \prod_{n \geq 1}\left(t^{n}+1\right)^{\psi_{m}^{-}(n) / n},
$$

where

$$
g_{m}(t):= \begin{cases}(t-1) & \text { if } \operatorname{gcd}(m, 6)=1 \\ (t+1)^{-1} & \text { if } \operatorname{gcd}(m, 6)=2 ; \\ \left(t^{3}-1\right)(t-1) & \text { if } \operatorname{gcd}(m, 6)=3 \\ \left(t^{3}+1\right)^{-1}(t+1)^{-1} & \text { if } \operatorname{gcd}(m, 6)=6\end{cases}
$$

Remark. Note that all but finitely many factors in the right-hand side of (4) are equal to one.

Proof. First, note that Theorem 3.2 and Proposition 3.3 yield that the degree $D_{m}$ of the polynomial $P_{m}(t)$ satisfies $D_{m}=\left|R_{m}\right|-(-1)^{m} \cdot \theta_{m}^{2}$, where $\theta_{m}$ is defined as in (1) in Section 1.3. Since $\sum_{n}\left(\psi_{m}^{+}(n)+\psi_{m}^{-}(n)\right)=\left|R_{m}\right|$, the degree of the right-hand side of (4) is $D_{m}$ as desired. 
Note that

$$
D_{m}+\sum_{n \geq 1} \frac{Z\left(\Sigma_{m, n}\right)}{t^{n}}=\sum_{n \geq 0} \frac{\operatorname{Tr}\left(T_{m}^{n}\right)}{t^{n}}=\frac{t P_{m}^{\prime}(t)}{P_{m}(t)}
$$

the second equality is true for any matrix. Writing $\theta_{m, n}:=\theta_{\operatorname{gcd}(m, n)}$ and applying Theorem 1.2 , we conclude that it suffices to prove that

$$
\begin{aligned}
& D_{m}+\sum_{n \geq 1} \frac{-(-1)^{\operatorname{gcd}(m, n)} \theta_{m, n}^{2}+\left|R_{m, n}^{+}\right|-\left|R_{m, n}^{-}\right|}{t^{n}} \\
= & \frac{t g_{m}^{\prime}(t)}{g_{m}(t)}+\sum_{n \geq 1} \frac{\psi_{m}^{+}(n) t^{n}}{t^{n}-1}+\sum_{n \geq 1} \frac{\psi_{m}^{-}(n) t^{n}}{t^{n}+1} .
\end{aligned}
$$

Now, by construction, we have that

$$
\begin{aligned}
& \sum_{n \geq 1} \frac{\left|R_{m, n}^{+}\right|-\left|R_{m, n}^{-}\right|}{t^{n}}=\sum_{n \geq 1} \frac{\psi_{m}^{+}(n)}{t^{n}-1}-\sum_{n \geq 1} \frac{\psi_{m}^{-}(n)}{t^{n}+1} \\
= & \sum_{n \geq 1}\left(\frac{\psi_{m}^{+}(n) t^{n}}{t^{n}-1}-\psi_{m}^{+}(n)\right)+\sum_{n \geq 1}\left(\frac{\psi_{m}^{-}(n) t^{n}}{t^{n}+1}-\psi_{m}^{-}(n)\right) \\
= & \sum_{n \geq 1} \frac{\psi_{m}^{+}(n) t^{n}}{t^{n}-1}+\sum_{n \geq 1} \frac{\psi_{m}^{-}(n) t^{n}}{t^{n}+1}-D_{m}-(-1)^{m} \theta_{m}^{2} .
\end{aligned}
$$

Thus it remains to prove that

$$
\frac{t g_{m}^{\prime}(t)}{g_{m}(t)}=\sum_{n \geq 0} \frac{-(-1)^{\operatorname{gcd}(m, n)} \theta_{m, n}^{2}}{t^{n}}
$$

This is a straightforward exercise in all four cases; hence (4) follows.

Proof of Conjecture 2. Let $\rho$ and $\rho^{\prime}$ be the rhombus tilings in Figure 8. One easily checks that $\rho \in R_{2 k}^{+}$and $\rho^{\prime} \in R_{6 k}^{+}$for each integer $k$ and also that $\nu(\rho)=4$ and $\nu\left(\rho^{\prime}\right)=6$. In particular, for $m$ such that $\operatorname{gcd}(m, 6)=2$, the right-hand side of (4) contains a factor $t^{4}-1$. Multiplying with $g_{m}(t)=(t+1)^{-1}$, we obtain $\left(t^{2}-1\right)(t-1)$. For $m$ such that $\operatorname{gcd}(m, 6)=6$, the right-hand side of $(4)$ contains a factor $\left(t^{4}-1\right)\left(t^{6}-1\right)$. This time, multiplication with $g_{m}(t)=(t+1)^{-1}\left(t^{3}+1\right)^{-1}$ yields $\left(t^{2}-1\right)(t-1)\left(t^{3}-1\right)$. To see that every factor $t^{s} \pm 1$ in $P_{m}(t)$ (except the one linear term $t-1$ ) satisfies $\operatorname{gcd}(s, m) \neq 1$, simply note that we cannot have $\nu(\rho)=n$ if $\rho \in R_{m}$ and $\operatorname{gcd}(m, n)=1$; apply Theorem 2.7.

\section{Properties of translation permutations}

Recall that $\mathbf{s}(x)=x+(1,0), \mathrm{e}(x)=x+(0,1)$, and $V_{u, v}=\mathbb{Z}^{2} /\langle u, v\rangle$. We present basic facts about translation permutations of the form $\mathbf{s}^{a} \mathrm{e}^{b}: V_{u, v} \rightarrow V_{u, v}$. We do this in preparation for our proof of Theorem 1.2 in Section 5. Before proceeding, let us introduce some conventions for figures, which will be used throughout the remainder of the paper. 


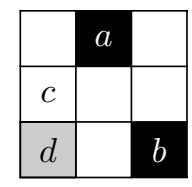

Figure 9: A subset $\sigma$ of $V_{u, v}$ restricted to a $3 \times 3$ piece of $S_{u, v}$. The black squares $a$ and $b$ belong to $\sigma$, the white square $c$ does not belong to $\sigma$, and the status is unknown or unimportant for the gray square $d$. All other squares in the figure are blocked by $a$ or $b$ and do not belong to $\sigma$ if $\sigma \in \Sigma\left(S_{u, v}\right)$.

We identify each point in $\mathbb{Z}^{2}$ with a unit square; two vertices being joined by an edge means that the corresponding squares share a common side. In any picture illustrating a subset $\sigma$ of $V_{u, v}$ restricted to a given piece of $S_{u, v}$, the following conventions apply for a given vertex $x$ :

- $x \in \sigma$ : the square representing $x$ is black.

- $x \notin \sigma$ : the square is white.

- The status of $x$ is unknown or unimportant: the square is gray.

See Figure 9 for an example.

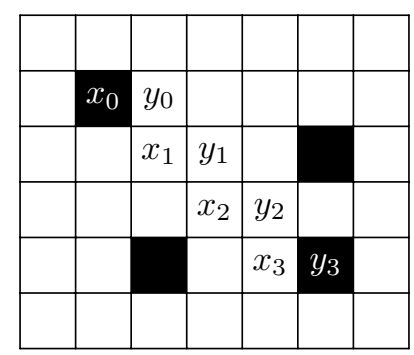

Figure 10: For $\pi=$ se, the elements $x_{0}, x_{1}, x_{2}$, and $y_{3}$ are $(\pi, \sigma)$-free, because they are all unblocked, $x_{0}, y_{3} \in \sigma$, and $\pi^{-2}\left(x_{2}\right)=\pi^{-1}\left(x_{1}\right)=x_{0}$. However, $x_{3}, y_{0}, y_{1}$, and $y_{2}$ are not $(\pi, \sigma)$-free; $x_{3}$ and $y_{0}$ are blocked by $y_{3}$ and $x_{0}$, respectively, whereas $\pi^{-2}\left(y_{2}\right)=\pi^{-1}\left(y_{1}\right)=$ $y_{0}$.

Let $\sigma$ be a set in $\Sigma_{u, v}:=\Sigma\left(S_{u, v}\right)$. For a permutation $\pi: V_{u, v} \rightarrow V_{u, v}$ and an element $y \in V_{u, v}$, let $\xi:=\xi_{\pi, \sigma}(y) \geq 0$ be minimal such that $\pi^{-\xi}(y) \in \sigma$; note the minus sign. If no such $\xi$ exists, we define $\xi_{\pi, \sigma}(y):=\infty$. We say that $y$ is $(\pi, \sigma)$-free if $\xi_{\pi, \sigma}(y)<\infty$ and all elements in the set $\left\{y, \pi^{-1}(y), \ldots, \pi^{-\xi_{\pi, \sigma}(y)}(y)\right\}$ are unblocked in $\sigma$; hence $\sigma+z \in \Sigma_{u, v}$ whenever $z$ belongs to this set. See Figure 10 for an example.

Lemma 4.1. Let $\pi: V_{u, v} \rightarrow V_{u, v}$ be a permutation and let $\sigma \in \Sigma_{u, v}$. If $y$ is $(\pi, \sigma)$-free, then $\pi^{-k}(y)$ is $(\pi, \sigma)$-free whenever $k \leq \xi_{\pi, \sigma}(y)$. 
Proof. This is immediate from the definition.

Lemma 4.2. Let $\pi:=\mathrm{s}^{a} \mathrm{e}^{b}$ be a translation permutation and let $\sigma \in \Sigma_{u, v}$. Then the set $\pi^{*}(\sigma)$ of $(\pi, \sigma)$-free elements belongs to $\Sigma_{u, v}$.

Proof. Assume the opposite; $\pi^{*}(\sigma)$ contains two neighbors $x$ and $y$. Write $\xi_{\sigma}:=\xi_{\pi, \sigma}$. By construction, $\pi^{-r}(x)$ and $\pi^{-s}(y)$ are $(\pi, \sigma)$-free whenever $r \leq \xi_{\sigma}(x)$ and $s \leq \xi_{\sigma}(y)$. Now, $\pi^{-\xi_{\sigma}(y)}(x)$ is blocked by $\pi^{-\xi_{\sigma}(y)}(y)$ in $\sigma$, which implies that $\xi_{\sigma}(x)<\xi_{\sigma}(y)$. However, we also have that $\pi^{-\xi_{\sigma}(x)}(y)$ is blocked by $\pi^{-\xi_{\sigma}(x)}(x)$ in $\sigma$, which implies that $\xi_{\sigma}(y)<\xi_{\sigma}(x)$. This is a contradiction.

Lemma 4.3. Let $\pi:=\mathrm{s}^{a} \mathrm{e}^{b}$ be a nontrivial translation permutation and let $\sigma \in \Sigma_{u, v}$. Let $x, y$ be $(\pi, \sigma)$-free elements such that $y=\pi(x)$ and $x \in \sigma$. Then an element $z$ is $(\pi, \sigma-y)$-free if and only if $z$ is $(\pi, \sigma+y)$-free.

Proof. Clearly, $\sigma+y \in \Sigma_{u, v}$. Moreover, $y$ is $(\pi, \sigma-y)$-free, because $y$ is unblocked and $y=\pi(x)$, which belongs to $\sigma$.

Now, let $z$ be some element outside $\sigma+y$. First, suppose that $z$ is $(\pi, \sigma+y)$-free but not $(\pi, \sigma-y)$-free. This implies that $\xi_{\sigma+y}(z) \neq \xi_{\sigma-y}(z)$. The only possibility is that $y=\pi^{-\xi_{\sigma+y}(z)}(z)$, which implies that $\xi_{\sigma-y}(z)=\xi_{\sigma+y}(z)+1$, because $x \in \sigma$. However, since $y$ is unblocked, this means that $z$ is $(\pi, \sigma-y)$-free, which is a contradiction.

Next, suppose that $z$ is $(\pi, \sigma-y)$-free but not $(\pi, \sigma+y)$-free. This means that $y$ is blocking some element $\pi^{-k}(z)$ such that $k \leq \xi_{\sigma+y}(z)$. However, since $\xi_{\sigma+y}(z) \leq \xi_{\sigma-y}(z)$, we have that $\pi^{-k}(z)$ is $(\pi, \sigma-y)$-free by Lemma 4.1. By Lemma 4.2, it follows that $y$ and $\pi^{-k}(z)$ are not neighbors, as both elements are $(\pi, \sigma-y)$-free. This is another contradiction.

Lemma 4.4. Let $(a, b)$ be an integer vector. Define

$$
d:=\operatorname{gcd}\left(b u_{1}-a u_{2}, b v_{1}-a v_{2},\left|V_{u, v}\right|\right) .
$$

Then the exponent of the translation permutation $\mathbf{s}^{a} \mathrm{e}^{b}: V_{u, v} \rightarrow V_{u, v}$ equals $\left|V_{u, v}\right| / d$.

Proof. We have that $\left(\mathrm{s}^{a} \mathrm{e}^{b}\right)^{k}$ is the identity if and only if there are integers $\lambda, \mu$ such that

$$
\lambda \cdot\left(u_{1}, u_{2}\right)+\mu \cdot\left(v_{1}, v_{2}\right)=k \cdot(a, b) .
$$

This implies that

$$
\lambda \cdot\left(b u_{1}-a u_{2}\right)+\mu \cdot\left(b v_{1}-a v_{2}\right)=0 .
$$

Write $d_{0}:=\operatorname{gcd}\left(b u_{1}-a u_{2}, b v_{1}-a v_{2}\right)$. We have that $(\lambda, \mu)$ is an integer solution to (6) if and only if $\lambda=-c\left(b v_{1}-a v_{2}\right) / d_{0}$ and $\mu=c\left(b u_{1}-a u_{2}\right) / d_{0}$ for some integer $c$. We obtain that

$$
k \cdot(a, b)=c \cdot \frac{u_{1} v_{2}-u_{2} v_{1}}{d_{0}} \cdot(a, b)= \pm c \cdot \frac{\left|V_{u, v}\right|}{d_{0}} \cdot(a, b) .
$$

As a consequence, the given $\lambda$ and $\mu$ yield a solution to (5) if and only if $c$ is an integer multiple of $d_{0} / \operatorname{gcd}\left(d_{0},\left|V_{u, v}\right|\right)=d_{0} / d$, which is equivalent to $k$ being an integer multiple of $\left|V_{u, v}\right| / d$.

Remark. One easily checks that $\operatorname{gcd}\left(u_{1}-u_{2}, v_{1}-v_{2},\left|V_{u, v}\right|\right)=\operatorname{gcd}\left(u_{1}-u_{2}, v_{1}-v_{2}\right)$ and $\operatorname{gcd}\left(u_{1}+u_{2}, v_{1}+v_{2},\left|V_{u, v}\right|\right)=\operatorname{gcd}\left(u_{1}+u_{2}, v_{1}+v_{2}\right)$. 


\section{Proof of Theorem 1.2}

The goal of this section is to prove Theorem 1.2, which we restate for convenience:

Write $d:=\operatorname{gcd}\left(u_{1}-u_{2}, v_{1}-v_{2}\right)$ and $d^{*}:=\operatorname{gcd}\left(u_{1}+u_{2}, v_{1}+v_{2}\right)$. Then

$$
Z\left(\Sigma_{u, v}\right)=-(-1)^{d} \theta_{d} \theta_{d^{*}}+\left|R_{u, v}^{+}\right|-\left|R_{u, v}^{-}\right|,
$$

where $\theta_{d}$ is defined as in (1) in Section 1.3.

The proof approach is to define a matching on $\Sigma_{u, v} \backslash\{\emptyset\}$ such that every matched pair $\{\sigma, \tau\}$ cancels out, meaning that $|\sigma| \not \equiv|\tau|(\bmod 2)$. Counting the unmatched sets, taking into account the parity of the sets, we will obtain the desired formula. We stress that our matching is purely combinatorial in nature and does not admit a topological interpretation in the language of discrete Morse theory [5].

By symmetry, $Z\left(\Sigma_{u, v}\right)=Z\left(\Sigma_{u^{\prime}, v^{\prime}}\right)$, where $u^{\prime}=\left(u_{1},-u_{2}\right)$ and $v^{\prime}=\left(v_{1},-v_{2}\right)$. This will be of some help in Step 12 at the end of the proof.

We divide the proof of Theorem 1.2 into several steps. Since the vectors $u$ and $v$ will be the same throughout the proof, we suppress $u$ and $v$ from notation and write $\Sigma$ instead of $\Sigma_{u, v}$.

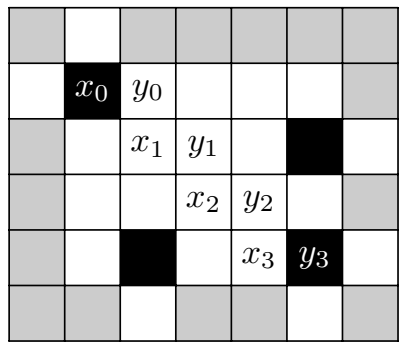

$\sigma$

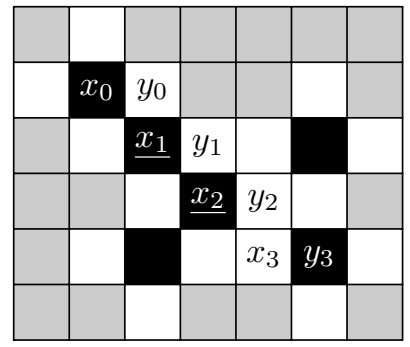

$(\mathrm{se})^{*}(\sigma)$

Figure 11: $x_{1}$ and $x_{2}$ belong to $(\mathrm{se})^{*}(\sigma)$; they are both unblocked and $(\mathrm{se})^{-2}\left(x_{2}\right)=$ $(\mathrm{se})^{-1}\left(x_{1}\right)=x_{0} \in \sigma$. However, $x_{3}, y_{0}, y_{1}$, and $y_{2}$ do not belong to $(\mathrm{se})^{*}(\sigma) ; x_{3}$ and $y_{0}$ are blocked, whereas $(\mathrm{se})^{-2}\left(y_{2}\right)=(\mathrm{se})^{-1}\left(y_{1}\right)=y_{0}$.

\section{Step 1: Partitioning $\Sigma$ into subfamilies $\Sigma^{0}$ and $\Delta$}

For a permutation $\pi$ and a set $\sigma \in \Sigma$, let $\pi^{*}(\sigma)$ be the set of $(\pi, \sigma)$-free elements. In this first step, we consider the set $(\mathrm{se})^{*}(\sigma)$; see Figure 11 for an illustration. Note that $(\mathrm{se})^{-1}=\mathrm{nw}$. We partition $\Sigma$ into two sets:

- $\Sigma^{0}$ is the subfamily of $\Sigma$ consisting of all $\sigma$ with the property that (se)* $(\sigma)$ contains an element $x$ such that $\mathrm{nw}(x) \in(\mathrm{se})^{*}(\sigma)$ and $(\mathrm{nw})^{2}(x) \notin(\mathrm{se})^{*}(\sigma)$.

- $\Delta=\Sigma \backslash \Sigma^{0}$. 


\section{Step 2: Getting rid of $\Sigma^{0}$}

For any set $X$, let $\Sigma^{0}(X)$ be the subfamily of $\Sigma^{0}$ consisting of all sets $\sigma$ such that $(\mathrm{se})^{*}(\sigma)=X$. We want to define a perfect matching on $\Sigma^{0}(X)$. Assume that this family is nonempty. In particular, there is an element $x$ in $X$ such that $\mathrm{nw}(x) \in X$ and $(\mathrm{nw})^{2}(x) \notin X$. To obtain the matching, note that $\mathrm{nw}(x) \in \sigma$ whenever $\sigma \in \Sigma^{0}(X)$, because $x$ is $(\mathbf{s e}, \sigma)$-free, whereas $(\mathrm{nw})^{2}(x)$ is not. By Lemma 4.3, we have that $(\mathbf{s e})^{*}(\sigma+x)=$ $(\mathrm{se})^{*}(\sigma-x)$, as $\mathrm{nw}(x) \in \sigma$. As a consequence, we obtain a perfect matching on $\Sigma^{0}(X)$ by pairing $\sigma-x$ and $\sigma+x$. We summarize:

Lemma 5.1. We have that $Z\left(\Sigma^{0}\right)=0$.

\section{Step 3: Proceeding with the remaining family $\Delta$}

It remains to consider the family $\Delta$ consisting of all sets $\sigma$ in $\Sigma$ with the property that $(\mathrm{se})^{*}(\sigma)$ does not contain any element $x$ such that $\mathrm{nw}(x) \in(\mathrm{se})^{*}(\sigma)$ and $(\mathrm{nw})^{2}(x) \notin$ $(\mathrm{se})^{*}(\sigma)$.

For a set $\sigma \in \Sigma$ and a permutation $\pi$, we say that an element $x \in \pi^{*}(\sigma)$ is $\pi$-cyclic in $\sigma$ if $\left\{\pi^{i}(x): i \in \mathbb{Z}\right\}$ is a subset of $\pi^{*}(\sigma)$; we refer to this subset as a $\pi$-cycle. Note that any $\pi$-cycle is also a $\pi^{-1}$-cycle, and vice versa. Let $\pi^{\infty}(\sigma)$ be the set of $\pi$-cyclic elements in $\pi^{*}(\sigma)$.

Lemma 5.2. Suppose that $\sigma \in \Delta$. If $x \in \sigma \backslash(\mathrm{se})^{\infty}(\sigma)$, then $\mathrm{s}^{2} \mathrm{e}(x) \in \sigma$ or $\mathrm{se}^{2}(x) \in \sigma$. Moreover, if $x \in \sigma \cap(\mathbf{s e})^{\infty}(\sigma)$, then the entire se-cycle $\left\{(\mathbf{s e})^{i}(x): i \in \mathbb{Z}\right\}$ is contained in $(\mathrm{se})^{*}(\sigma)$. Indeed, these two properties characterize $\Delta$ within $\Sigma$.

Proof. For the first part of the lemma, it suffices to prove that $y:=\operatorname{se}(x)$ is blocked in $\sigma$. Namely, since $x$ blocks $\mathrm{n}(y)=\mathrm{e}(x)$ and $\mathrm{w}(y)=\mathbf{s}(x)$, the element blocking $y$ must be either $\mathbf{s}(y)=\mathbf{s}^{2} \mathrm{e}(x)$ or $\mathrm{e}(y)=\mathbf{s e}^{2}(x)$. Let $k \geq 0$ be minimal such that $(\mathrm{nw})^{k+1}(x) \notin(\mathrm{se})^{*}(\sigma)$. Since $\sigma \in \Delta$, we have that $(\mathrm{nw})^{k-1}(x) \notin(\mathrm{se})^{*}(\sigma)$. The only possibility is that $k=0$, which settles the claim. The other statements in the lemma are obvious.

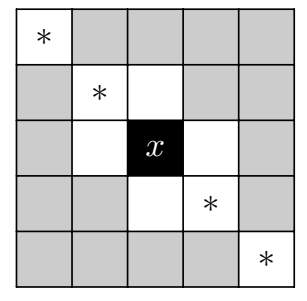

Figure 12: The situation around an element $x$ in $\left(\mathrm{n}^{3} \mathrm{w}^{3}\right)^{*}(\sigma)$, where $\sigma \in \Delta$ and $x \notin$ $(\mathrm{se})^{\infty}(\sigma)$. The elements marked with stars being absent is a consequence of Lemma 5.3.

For $\sigma \in \Delta$, we will consider the set $\left(\mathrm{n}^{3} \mathrm{w}^{3}\right)^{*}(\sigma)$ of $\left(\mathrm{n}^{3} \mathrm{w}^{3}, \sigma\right)$-free elements. Hence instead of going one step in the south-east direction, we go three steps in the opposite direction. 
Lemma 5.3. If $\sigma \in \Delta$ and $x \in\left(\mathrm{n}^{3} \mathrm{w}^{3}\right)^{*}(\sigma) \backslash(\mathrm{se})^{\infty}(\sigma)$, then the four elements $\mathrm{n}^{2} \mathrm{w}^{2}(x)$, $\mathrm{nw}(x)$, se $(x)$, and $\mathrm{s}^{2} \mathrm{e}^{2}(x)$ do not belong to $\left(\mathrm{n}^{3} \mathrm{w}^{3}\right)^{*}(\sigma)$; see Figure 12.

Proof. Let $x$ be as in the lemma. First, suppose that there is an element $y$ in $\left(\mathrm{n}^{3} \mathrm{w}^{3}\right)^{*}(\sigma) \cap$ $\left\{\mathrm{n}^{2} \mathrm{w}^{2}(x), \mathrm{nw}(x)\right\}$. We claim that this implies that $\mathrm{s}^{3} \mathrm{e}^{3}(y) \in\left(\mathrm{n}^{3} \mathrm{w}^{3}\right)^{*}(\sigma)$; we refer to this as "property A". To prove the claim, assume to the contrary that $\mathrm{s}^{3} \mathrm{e}^{3}(y) \notin\left(\mathrm{n}^{3} \mathrm{w}^{3}\right)^{*}(\sigma)$. This implies that $y \in \sigma$; hence either $\operatorname{se}^{2}(y)$ or $\mathrm{s}^{2} \mathrm{e}(y)$ belongs to $\sigma$ by Lemma 5.2. However, this is a contradiction to Lemma 4.2 , because both these elements are blocked by $x$.

We use induction on $\xi(x):=\xi_{\left(\mathrm{n}^{3} \mathrm{w}^{3}\right)^{*}, \sigma}(x)$ to prove the lemma. If $\xi(x)=0$, meaning that $x \in \sigma$, then Lemma 5.2 yields that either $\mathrm{se}^{2}(x)$ or $\mathrm{s}^{2} \mathrm{e}(x)$ belongs to $\sigma$. In particular, $\mathrm{se}(x)$ and $\mathrm{s}^{2} \mathrm{e}^{2}(x)$ are both blocked and hence not present in $\left(\mathrm{n}^{3} \mathrm{w}^{3}\right)^{*}(\sigma)$. By property A, $\mathrm{n}^{3} \mathrm{w}^{3}(\mathrm{se}(x))=\mathrm{n}^{2} \mathrm{w}^{2}(x)$ and $\mathrm{n}^{3} \mathrm{w}^{3}\left(\mathrm{~s}^{2} \mathrm{e}^{2}(x)\right)=\mathrm{nw}(x)$ are not present either.

If $\xi(x)>0$, then $x^{\prime}:=\mathrm{s}^{3} \mathrm{e}^{3}(x) \in\left(\mathrm{n}^{3} \mathrm{w}^{3}\right)^{*}(\sigma)$. Since $\xi\left(x^{\prime}\right)=\xi(x)-1$, induction yields that $\mathrm{n}^{2} \mathrm{w}^{2}\left(x^{\prime}\right)=\mathrm{se}(x)$ and $\mathrm{nw}\left(x^{\prime}\right)=\mathrm{s}^{2} \mathrm{e}^{2}(x)$ do not belong to $\left(\mathrm{n}^{3} \mathrm{w}^{3}\right)^{*}(\sigma)$. Another application of property $\mathrm{A}$ settles the lemma.

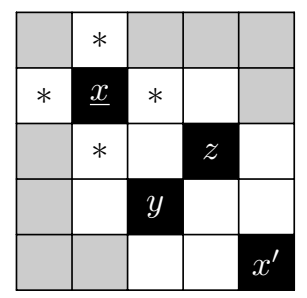

Figure 13: The situation in Lemma 5.4. Given that $x^{\prime}, y, z \in\left(\mathrm{n}^{3} \mathrm{w}^{3}\right)^{*}(\sigma)$, the elements marked with stars do not belong to $\left(\mathrm{n}^{3} \mathrm{w}^{3}\right)^{*}(\sigma)$; this is because of Lemma 5.3. It follows that the underlined element $x$ belongs to $\left(\mathrm{n}^{3} \mathrm{w}^{3}\right)^{*}(\sigma)$.

Lemma 5.4. Suppose that $\sigma \in \Delta$ and $\mathrm{s}^{3} \mathrm{e}^{3}(x), \mathrm{s}^{2} \mathrm{e}(x), \mathrm{se}^{2}(x) \in\left(\mathrm{n}^{3} \mathrm{w}^{3}\right)^{*}(\sigma)$. Then $x \in$ $\left(\mathrm{n}^{3} \mathrm{w}^{3}\right) *(\sigma)$.

Proof. Write $y:=\mathrm{s}^{2} \mathrm{e}(x), z:=\mathrm{se}^{2}(x)$, and $x^{\prime}:=\mathrm{s}^{3} \mathrm{e}^{3}(x)$. Clearly, these elements do not belong to $\mathrm{se}^{\infty}(\sigma)$. Since $x^{\prime} \in\left(\mathrm{n}^{3} \mathrm{w}^{3}\right)^{*}(\sigma), x^{\prime}$ is $\left(\mathrm{n}^{3} \mathrm{w}^{3}, \sigma\right)$-free. In particular, $x$ is free unless $x$ is blocked in $\sigma$. However, applying Lemma 5.3 to $y$, we obtain that $\mathrm{s}(x)$ and $\mathrm{w}(x)$ do not belong to $\sigma$, whereas the same lemma applied to $z$ yields that $\mathrm{e}(x)$ and $\mathrm{n}(x)$ do not belong to $\sigma$. Hence $x$ is not blocked in $\sigma$, and we are done. See Figure 13 for an illustration.

Lemma 5.5. If $\sigma \in \Delta$ and $x \in\left(\mathrm{n}^{3} \mathrm{w}^{3}\right)^{*}(\sigma) \backslash(\mathrm{se})^{\infty}(\sigma)$, then the following hold:

- Either $\mathrm{n}^{2} \mathrm{w}(x)$ or $\mathrm{nw}^{2}(x)$ belongs to $\left(\mathrm{n}^{3} \mathrm{w}^{3}\right)^{*}(\sigma)$.

- Either $\mathrm{s}^{2} \mathrm{e}(x)$ or $\mathrm{se}^{2}(x)$ belongs to $\left(\mathrm{n}^{3} \mathrm{w}^{3}\right)^{*}(\sigma)$. 
Proof. First, suppose that the first statement is true and the second statement is false. Let $x \in\left(\mathrm{n}^{3} \mathrm{w}^{3}\right)^{*}(\sigma) \backslash(\mathrm{se})^{\infty}(\sigma)$ be such that $\mathrm{s}^{2} \mathrm{e}(x)$ and $\mathrm{se}^{2}(x)$ do not belong to $\left(\mathrm{n}^{3} \mathrm{w}^{3}\right)^{*}(\sigma)$. By Lemma 5.2, $x \in\left(\mathrm{n}^{3} \mathrm{w}^{3}\right)^{*}(\sigma) \backslash \sigma$, which yields that $x^{\prime}:=\mathrm{s}^{3} \mathrm{e}^{3}(x) \in\left(\mathrm{n}^{3} \mathrm{w}^{3}\right)^{*}(\sigma)$. However, $\mathrm{nw}^{2}\left(x^{\prime}\right)=\mathrm{s}^{2} \mathrm{e}(x)$ and $\mathrm{n}^{2} \mathrm{w}\left(x^{\prime}\right)=\mathrm{se}^{2}(x)$, which contradicts the assumption that the first statement is true.

It hence suffices to prove the first statement. Refer to an element contradicting this statement as a bad element. Let $x$ be a bad element and let $k$ be minimal such that $x_{2 k}^{\prime}:=$ $\left(\mathrm{s}^{3} \mathrm{e}^{3}\right)^{k}(x) \in \sigma$. We may assume that $x_{2 i}^{\prime}:=\left(\mathbf{s}^{3} \mathrm{e}^{3}\right)^{i}(x)$ is not bad for $1 \leq i \leq k$; otherwise, replace $x$ with $x_{2 i}^{\prime}$, where $i$ is maximal such that $x_{2 i}^{\prime}$ is bad. In particular, for each $i$ such that $0 \leq i \leq k-1$, we have an element $x_{2 i+1}^{\prime}$ such that $x_{2 i+1}^{\prime} \in\left\{\operatorname{se}^{2}\left(x_{2 i}^{\prime}\right), \mathrm{s}^{2} \mathrm{e}\left(x_{2 i}^{\prime}\right)\right\}$.

Now, for each $y \in \sigma$, either $\mathrm{s}^{2} \mathrm{e}(y)$ or $\mathrm{se}^{2}(y)$ belongs to $\sigma$. In particular, by the construction of $x_{1}^{\prime}, \ldots, x_{2 k}^{\prime}$ above and by the fact that $x_{2 k}^{\prime} \in \sigma$, there is an infinite sequence $\left(x_{0}=x, x_{1}, x_{2}, x_{3}, \ldots\right)$ such that $x_{i} \in\left(\mathrm{n}^{3} \mathrm{w}^{3}\right)^{*}(\sigma)$ and $x_{i} \in\left\{\mathrm{s}^{2} \mathrm{e}\left(x_{i-1}\right), \mathrm{se}^{2}\left(x_{i-1}\right)\right\}$ for all $i \geq 1$. Note that $x_{i}$ is not necessarily equal to $x_{i}^{\prime}$ for $1 \leq i \leq 2 k$; we defined the elements $x_{i}^{\prime}$ just to be able to deduce that there is some infinite sequence.

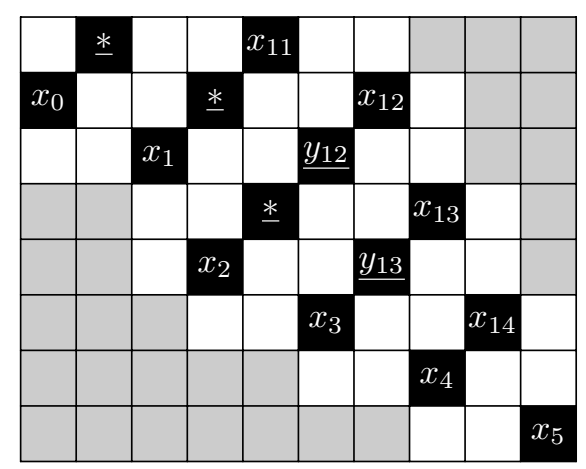

$$
x_{11}=x_{r}
$$

$$
\begin{aligned}
& x_{4}=y_{14} \\
& x_{5}=x_{j}=x_{k}
\end{aligned}
$$

Figure 14: The construction in the proof of Lemma 5.5 with $(j, k, r)=(5,15,11)$. By the proof, $y_{12}, y_{13} \in\left(\mathrm{n}^{3} \mathrm{w}^{3}\right)^{*}(\sigma)$. We obtain a path from $x_{11}=x_{r}$ to $x_{4}=x_{j-1}$ and hence a cycle through $x_{j-1}$, which contradicts the minimality of $j$. One may proceed to prove that the elements marked with stars also belong to $\left(\mathrm{n}^{3} \mathrm{w}^{3}\right)^{*}(\sigma)$.

We illustrate the following construction in Figure 14. Let $j \geq 0$ be minimal such that $x_{j}=x_{k}$ for some $k>j$. We assume that we have chosen the sequence $\left\{x_{i}: i \geq 0\right\}$ such that $j$ is as small as possible.

To prove the lemma, it suffices to show that $j=0$. Namely, $x_{k-1}$ will then be an element contradicting the assumption about $\mathrm{n}^{2} \mathrm{w}(x)$ and $\mathrm{nw}^{2}(x)$ not belonging to $\left(\mathrm{n}^{3} \mathrm{w}^{3}\right)^{*}(\sigma)$. Assume to the contrary that $j>0$. We have that $x_{j-1}$ and $x_{k-1}$ are distinct by the minimality of $j$. There are two possibilities:

- $x_{j-1}=\mathrm{nw}^{2}\left(x_{k}\right)$ and $x_{k-1}=\mathrm{n}^{2} \mathrm{w}\left(x_{k}\right)$. Let $r \leq k-2$ be maximal such that $x_{r}=$ $\mathrm{nw}^{2}\left(x_{r+1}\right)$; note that $r \geq j-1$. We claim that $y_{i}:=\mathrm{nw}^{2}\left(x_{i+1}\right) \in\left(\mathrm{n}^{3} \mathrm{w}^{3}\right)^{*}(\sigma)$ for $i=$ $r+1, \ldots, k-1$. This is clear for $i=k-1$, because $y_{k-1}=x_{j-1}$. For $i<k-1$, assume inductively that $y_{i+1} \in\left(\mathrm{n}^{3} \mathrm{w}^{3}\right)^{*}(\sigma)$. Since $\mathrm{se}^{2}\left(y_{i}\right)=x_{i+1}, \mathrm{~s}^{3} \mathrm{e}^{3}\left(y_{i}\right)=\mathrm{s}^{2} \mathrm{e}\left(x_{i+1}\right)=$ 
$x_{i+2}$, and $\mathrm{s}^{2} \mathrm{e}\left(y_{i}\right)=\mathrm{nw}^{2}\left(x_{i+2}\right)=y_{i+1}$, Lemma 5.4 implies that $y_{i} \in\left(\mathrm{n}^{3} \mathrm{w}^{3}\right)^{*}(\sigma)$, which settles the claim.

Now, we may form a new sequence by replacing $x_{i}$ with $y_{i}$ for $i=r+1, \ldots, k-1$. Since $y_{k-1}=x_{j-1}$, this contradicts the minimality of $j$. Hence we must have that $j=0$.

- $x_{j-1}=\mathrm{n}^{2} \mathrm{w}\left(x_{k}\right)$ and $x_{k-1}=\mathrm{nw}^{2}\left(x_{k}\right)$. By symmetry, this case is proved in exactly the same manner as the previous case.

Corollary 5.6. If $\sigma$ belongs to $\Delta$, then so does $\left(\mathrm{n}^{3} \mathrm{w}^{3}\right)^{*}(\sigma)$.

Proof. By Lemma 4.2, $\left(\mathrm{n}^{3} \mathrm{w}^{3}\right)^{*}(\sigma)$ belongs to $\Sigma$ whenever $\sigma$ belongs to $\Sigma$. It remains to prove that $\left(\mathrm{n}^{3} \mathrm{w}^{3}\right)^{*}(\sigma)$ belongs to $\Delta$ whenever $\sigma$ belongs to $\Delta$. By Lemma 5.2 , this is equivalent to saying the following: Whenever $x \in\left(\mathrm{n}^{3} \mathrm{w}^{3}\right)^{*}(\sigma) \backslash(\mathrm{se})^{\infty}(\sigma)$, at least one of the elements $\mathrm{s}^{2} \mathrm{e}(x)$ and $\mathrm{se}^{2}(x)$ belongs to $\left(\mathrm{n}^{3} \mathrm{w}^{3}\right)^{*}(\sigma)$. This is a consequence of Lemma 5.5.

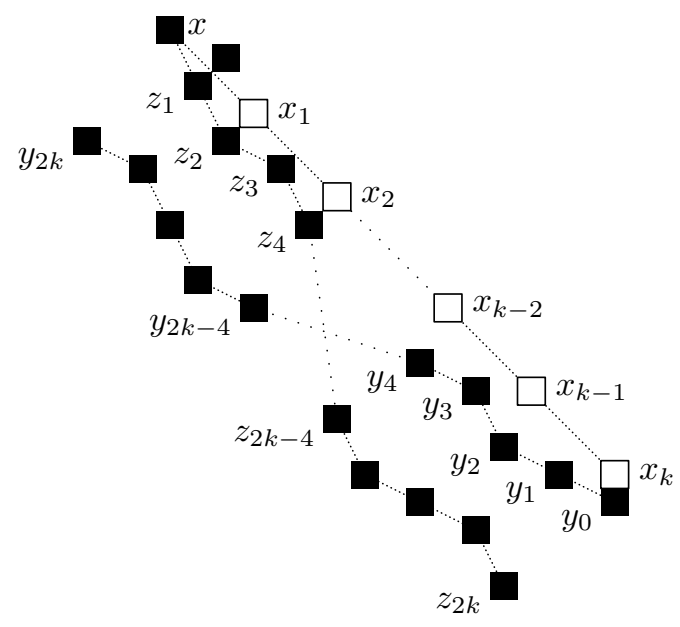

Figure 15: The construction in the proof of Lemma 5.7. The existence of elements $y_{2 k-i}$ and $z_{i}$ blocking each other is a consequence of the fact that the " $y$-path" and the "z-path" intersect.

Lemma 5.7. Suppose that $\sigma \in \Delta$ and $x, \mathrm{~s}^{2} \mathrm{e}(x), \mathrm{se}^{2}(x) \in\left(\mathrm{n}^{3} \mathrm{w}^{3}\right)^{*}(\sigma)$. Then $\mathrm{s}^{3} \mathrm{e}^{3}(x) \in$ $\left(\mathrm{n}^{3} \mathrm{w}^{3}\right) *(\sigma)$.

Proof. Assume the opposite. We illustrate the following construction in Figure 15. Let $k$ be minimal such that $x_{k}:=\left(\mathrm{s}^{3} \mathrm{e}^{3}\right)^{k}(x)$ is blocked in $\sigma$. Such a $k$ exists, because $x_{r}=x$ for sufficiently large $r$, and $x_{1}, \ldots, x_{r-1}$ cannot be all unblocked, as $x_{1} \notin\left(\mathrm{n}^{3} \mathrm{w}^{3}\right)^{*}(\sigma)$. For the same reason, $x_{2}, \ldots, x_{k} \notin\left(\mathrm{n}^{3} \mathrm{w}^{3}\right)^{*}(\sigma)$. Let $y \in \sigma$ be an element blocking $x_{k} ; y=\pi\left(x_{k}\right)$, 
where $\pi \in\{\mathrm{n}, \mathrm{w}, \mathrm{e}, \mathrm{s}\}$. By symmetry, we may assume that $\pi$ is either $\mathrm{s}$ or $\mathrm{w}$, meaning that $y$ is "below" the line through $x, x_{1}, \ldots, x_{k}$.

Let $z_{1}, z_{2}, \ldots, z_{2 k}$ be elements in $\left(\mathrm{n}^{3} \mathrm{w}^{3}\right)^{*}(\sigma)$ such that $z_{1}=\mathrm{s}^{2} \mathrm{e}(x)$ and such that $z_{i} \in$ $\left\{\mathrm{se}^{2}\left(z_{i-1}\right), \mathrm{s}^{2} \mathrm{e}\left(z_{i-1}\right)\right\}$ for all $i$; apply Lemma 5.5. Write $z_{i}=\mathrm{s}^{a_{i}} \mathrm{e}^{3 i-a_{i}}(x)$. Using induction, one easily proves that $a_{i}>3 i-a_{i}$ for $1 \leq i \leq 2 k$. Namely, if $z_{2 i-1}=\mathrm{s}^{3 i-1} \mathrm{e}^{3 i-2}(x)$, then we must have that $z_{2 i}=\mathbf{s}^{3 i+1} \mathrm{e}^{3 i-1}(x)$, because $\mathbf{s}^{3 i} \mathrm{e}^{3 i}(x)=x_{i}$ is not in $\left(\mathrm{n}^{3} \mathrm{w}^{3}\right)^{*}(\sigma)$ for $i \leq k$.

Now, let $y_{0}, y_{1}, y_{2}, \ldots, y_{k}$ be elements in $\left(\mathrm{n}^{3} \mathrm{w}^{3}\right)^{*}(\sigma)$ such that $y_{0}=y$ and such that $y_{i} \in\left\{\mathrm{nw}^{2}\left(y_{i-1}\right), \mathrm{n}^{2} \mathrm{w}\left(y_{i-1}\right)\right\}$ for all $i$; again, apply Lemma 5.5. Write $y_{i}=\mathrm{n}^{3 i-b_{i}} \mathrm{w}^{b_{i}}(y)$. As above, induction yields that $b_{i}>3 i-b_{i}$ for $1 \leq i \leq 2 k$. Namely, if $y_{2 i-1}=\mathrm{n}^{3 i-2} \mathrm{w}^{3 i-1}(y)$, then we must have that $y_{2 i}=\mathrm{n}^{3 i-1} \mathrm{w}^{3 i+1}(x)$, because $\mathrm{n}^{3 i} \mathrm{w}^{3 i}(x)$ blocks $x_{k-i}$. Moreover, $y_{1}=\mathrm{nw}^{2}\left(y_{0}\right)$, as $\mathrm{n}^{2} \mathrm{w}\left(\mathrm{n}^{2} \mathrm{w}\left(y_{0}\right)\right)=\mathrm{ne} \circ \pi\left(x_{k-1}\right)$ and $\mathrm{nw}^{2}\left(\mathrm{n}^{2} \mathrm{w}\left(y_{0}\right)\right)=\pi\left(x_{k-1}\right)$ both block $x_{k-1}$.

Let $i$ be such that

$$
2 a_{i}-3 i=2 b_{2 k-i}-3(2 k-i) \Longleftrightarrow a_{i}=b_{2 k-i}-3 k+3 i .
$$

Such an $i$ exists, because $\left(0,2 a_{1}-3,2 a_{2}-6, \ldots, 2 a_{2 k}-6 k\right)$ and $\left(0,2 b_{1}-3,2 b_{2}-6, \ldots, b_{2 k}-\right.$ $6 k$ ) both form sequences such that adjacent entries differ by exactly one and such that all entries are nonnegative. Now,

$$
\begin{aligned}
y_{2 k-i} & =\mathrm{n}^{6 k-3 i-b_{2 k-i}} \mathrm{~W}^{b_{2 k-i}}(y)=\mathrm{n}^{3 k-a_{i}} \mathrm{w}^{3 k-3 i+a_{i}}(y) \\
& =\mathbf{s}^{a_{i}-3 k} \mathrm{e}^{3 i-3 k-a_{i}}\left(\pi \circ \mathrm{s}^{3 k} \mathrm{e}^{3 k}(x)\right) \\
& =\pi \circ \mathrm{s}^{a_{i}} \mathrm{e}^{3 i-a_{i}}(x)=\pi\left(z_{i}\right) .
\end{aligned}
$$

Hence $y_{2 k-i}$ and $z_{i}$ block each other, which is a contradiction.

\section{Step 4: Partitioning $\Delta$ into subfamilies $\Gamma^{0}, \Gamma^{R}$, and $\Lambda$}

We consider $\mathrm{n}^{3} \mathrm{w}^{3}$-cycles, i.e., sets of the form $\left\{\left(\mathrm{n}^{3} \mathrm{w}^{3}\right)^{i}(x): i \geq 0\right\}$. We partition $\Delta$ into two subfamilies:

- $\Gamma$ consists of all $\sigma$ such that $\left(\mathrm{n}^{3} \mathrm{w}^{3}\right)^{*}(\sigma)$ is not a union of $\mathrm{n}^{3} \mathrm{w}^{3}$-cycles.

- $\Lambda$ consists of all $\sigma$ such that $\left(\mathrm{n}^{3} \mathrm{w}^{3}\right)^{*}(\sigma)$ is a union of $\mathrm{n}^{3} \mathrm{w}^{3}$-cycles.

In this and the following steps, we consider $\Gamma$; we postpone the treatment of $\Lambda$ until Step 7. We partition $\Gamma$ further into two subfamilies:

- $\Gamma^{0}$ is the family of all $\sigma \in \Gamma$ with the property that there is an element $x$ in $D:=\left(\mathrm{n}^{3} \mathrm{w}^{3}\right)^{*}(\sigma)$ such that $\mathrm{s}^{3} \mathrm{e}^{3}(x) \notin D$ and such that either of the following holds:

(1) $\mathrm{se}^{2}(x) \in D$ and $\mathrm{n}^{2} \mathrm{e}(x), \mathrm{ne}^{2}(x) \notin D$.

(2) $\mathrm{s}^{2} \mathrm{e}(x) \in D$ and $\mathrm{s}^{2} \mathrm{w}(x), \mathrm{sw}^{2}(x) \notin D$.

- $\Gamma^{R}=\Gamma \backslash \Gamma^{0}$.

See Figure 16 for an illustration. 


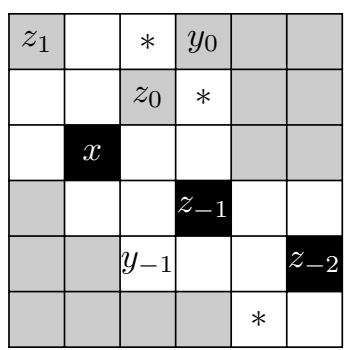

Figure 16: The situation in a set $\sigma$ around a vertex $x$ satisfying (1). Elements marked with stars are not in $\sigma$ by assumption. $z_{-1}, z_{-2} \in \sigma$ and $\mathrm{n}^{2}(x) \notin \sigma$ by Lemma $5.2 ; y_{-1} \notin \sigma$ by Lemma 5.7. Note that $z_{0}$ is unblocked.

\section{Step 5: Getting rid of $\Gamma^{0}$}

First, consider the family $\Gamma^{0}$. For any set $D$, let $\Gamma^{0}(D)$ be the family of sets $\sigma$ in $\Gamma^{0}$ such that $\left(\mathrm{n}^{3} \mathrm{w}^{3}\right)^{*}(\sigma)=D$.
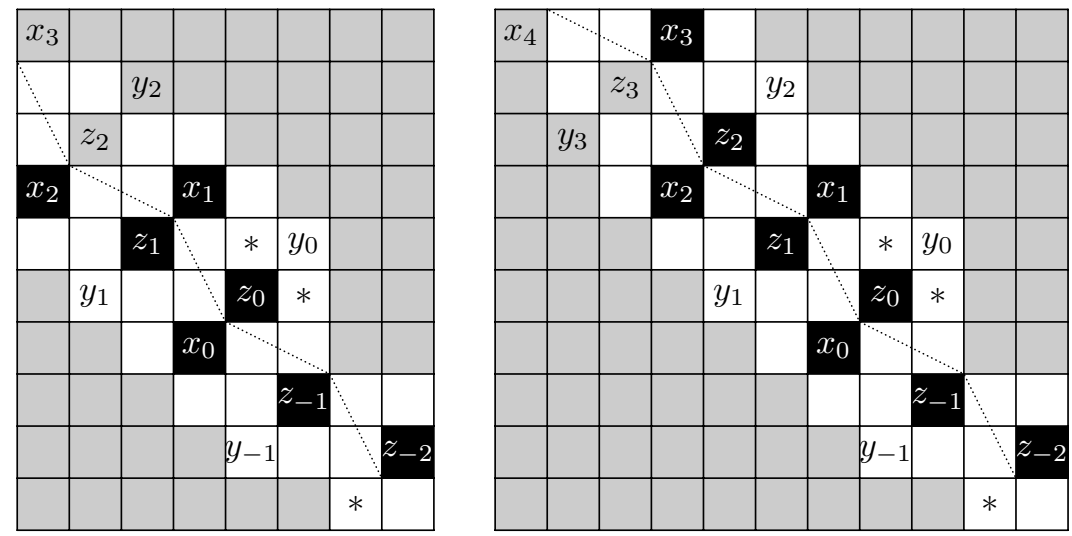

Figure 17: The cases $k=3$ (on the left) and $k=4$ (on the right) in the proof of Lemma 5.8. In both cases, either $x_{k} \notin \sigma$ or $y_{k-1} \in \sigma$. The dotted path indicates the symmetry of the construction.

Lemma 5.8. For each $D$, we have that $Z\left(\Gamma^{0}(D)\right)=0$. Hence $Z\left(\Gamma^{0}\right)=0$.

Proof. Assume that $\Gamma^{0}(D)$ is nonempty and let $\sigma \in \Gamma^{0}(D)$. Let $x$ be an element in $D$ such that $\mathrm{s}^{3} \mathrm{e}^{3}(x) \notin D$ and such that (1) or (2) holds. By symmetry, we may assume that (1) holds. Lemma 5.7 yields that we cannot have that $\mathrm{s}^{2} \mathrm{e}(x)$ and $\mathrm{se}^{2}(x)$ are both in $D$, as this would imply that $\mathrm{s}^{3} \mathrm{e}^{3}(x) \in D$. In particular, (1) and (2) are mutually exclusive. We obviously have that $x \in \sigma$. Moreover, Lemma 5.2 yields that $z_{-1}:=\mathrm{se}^{2}(x)$ and $z_{-2}:=\mathrm{s}^{2} \mathrm{e}^{4}(x)$ are both in $\sigma$.

We illustrate the following construction in Figure 17. For $i \geq 0$, define elements $x_{i}, y_{i}, z_{i}$ as follows: Let $x_{0}:=x, x_{1}:=\mathrm{n}^{3}(x)$, and $x_{i}=\mathrm{n}^{3} \mathrm{w}^{3}\left(x_{i-2}\right)$ for $i \geq 2$. Moreover, 
let $y_{2 i+1}=\mathrm{s}^{2} \mathrm{e}\left(x_{2 i+2}\right)$ and $y_{2 i}=\mathrm{se}^{2}\left(x_{2 i+1}\right)$ for $i \geq 0$. We also define $y_{-1}:=\mathrm{s}^{2} \mathrm{e}\left(x_{0}\right)$; this element is not present in $D$. Finally, let $z_{2 i}=\mathrm{ne}\left(x_{2 i}\right)$ and $z_{2 i+1}=\operatorname{sw}\left(x_{2 i+1}\right)$. Note that the definitions of $z_{-1}$ and $z_{-2}$ align well with the definition of $z_{i}$ for $i \geq 0$; see Figure 17 .

Let $k:=k_{\sigma} \geq 1$ be minimal such that either of the following holds:

- $x_{k} \notin \sigma$.

- $y_{k-1} \in \sigma$.

Such a $k$ exists, because $x_{j}=\mathrm{s}^{3} \mathrm{e}^{3}\left(x_{0}\right)$ for some $j$. Note that Lemma 5.2 applied to $x_{i+1}$ implies that $z_{i} \in \sigma$ for $0 \leq i \leq k-2$; this is because $y_{i} \notin \sigma$. Since before, we know that $z_{-1}$ and $z_{-2}$ belong to $\sigma$.

We want to prove the following:

(i) $\sigma-z_{k_{\sigma}-1}$ and $\sigma+z_{k_{\sigma}-1}$ both belong to $\Gamma^{0}(D)$.

(ii) $k_{\sigma}$ remains unchanged when $z_{k_{\sigma}-1}$ is added to or deleted from $\sigma$.

This will yield the lemma, because we may then form a perfect matching on $\Gamma^{0}(D)$ by pairing $\sigma-z_{k_{\sigma}-1}$ with $\sigma+z_{k_{\sigma}-1}$.

To prove (i), we first show that the two sets belong to $\Delta$. Write $k:=k_{\sigma}$. First, assume that $z_{k-1} \notin \sigma$ and $\sigma \in \Gamma^{0}(D)$. We have that $z_{k-1}$ is not blocked in $\sigma$. For $k=1$, this is by assumption. For $k \geq 2$, two of the neighbors of $z_{k-1}$ are also neighbors of $x_{k-1}$ and hence blocked, whereas the other two neighbors are $\mathrm{nw}\left(x_{k-2}\right)$ and $\mathrm{n}^{2} \mathrm{w}^{2}\left(x_{k-2}\right)$. By Lemma 5.3, these two elements are not present in $\sigma$. Moreover, thanks to $x_{k-2}$, Lemma 5.2 is true for $z_{k-1}$ in $\sigma+z_{k-1}$. Next, assume that $z_{k-1} \in \sigma$ and $\sigma \in \Gamma^{0}(D)$. If $\sigma-z_{k-1}$ is not in $\Delta$, then either we have that $z_{k} \in \sigma$ and $x_{k-1} \notin \sigma$ or we have that $x_{k} \in \sigma$ and $y_{k-1} \notin \sigma$. By construction, neither of these conditions can hold.

To conclude the proof of (i), note that $z_{k-3}$ is in $D$; this is true also for $k \leq 2$. In particular, to prove that $\left(\mathrm{n}^{3} \mathrm{w}^{3}\right)^{*}\left(\sigma-z_{k-1}\right)=\left(\mathrm{n}^{3} \mathrm{w}^{3}\right)^{*}\left(\sigma+z_{k-1}\right)$, it suffices to show that $z_{k-1}$ is unblocked in $\sigma$, which we did above.

It remains to prove (ii). Since $k:=k_{\sigma}$ is defined in terms of $\left\{x_{j}, y_{j-1}, j \geq 1\right\}$, things may go wrong only if $z_{k-1}=x_{j}$ or $z_{k-1}=y_{j-1}$ for some $j \in[1, k]$. Since $z_{k-1}-x_{k}=$ $y_{k-2}-x_{k-1}$, we have that $z_{k-1}=x_{k}$ if and only if $y_{k-2}=x_{k-1}$, which is impossible. Similarly, $z_{k-1}=y_{k-1}$ if and only if $y_{k-2}=z_{k-2}$, which is again impossible. It remains to consider the case $j<k$.

First, suppose that $z_{k-1}$ is equal to $x_{j}$ for some $j<k$. For $j=0$ and $k=1$, we have that $z_{0}=x_{0}$ if and only if $z_{-1}=y_{-1}$, which is a contradiction, as $z_{-1} \in \sigma$ and $y_{-1} \notin \sigma$. For $k \geq 2$, we have that $\left\{\mathrm{s}^{2} \mathrm{e}\left(z_{k-1}\right), \mathrm{se}^{2}\left(z_{k-1}\right)\right\}=\left\{z_{k-2}, x_{k-2}\right\} \subset \sigma$. Yet, either $\mathrm{s}^{2} \mathrm{e}\left(x_{j}\right)$ or $\mathrm{se}^{2}\left(x_{j}\right)$ equals $y_{j-1}$, which is not in $\sigma$, a contradiction.

Next, suppose that $z_{k-1}$ is equal to $y_{j-1}$ for some $j<k$. If $j \geq 2$, then $\mathrm{s}^{3} \mathrm{e}^{3}\left(y_{j-1}\right)=$ $y_{j-3}$, which is not in $\sigma$, contradicting the fact that $\mathrm{s}^{3} \mathrm{e}^{3}\left(z_{k-1}\right)=z_{k-3} \in \sigma$. Suppose that $j=1$; hence $z_{k-1}=y_{0}$. If $k$ is odd, then $\mathbf{s}^{3}\left(z_{k-1}\right)=y_{k-2} \notin \sigma$. However, $\mathbf{s}^{3}\left(y_{0}\right)=z_{-1} \in \sigma$, which is a contradiction. If $k$ is even, then $\mathbf{s}^{4} \mathrm{e}^{2}\left(z_{k-1}\right)=y_{k-3} \notin \sigma$. However, $\mathbf{s}^{4} \mathrm{e}^{2}\left(y_{0}\right)=$ $z_{-2} \in \sigma$, another contradiction. This concludes the proof of (ii), and we are done. 


\section{Step 6: Relating $\Gamma^{R}$ to a family of rhombus tilings}
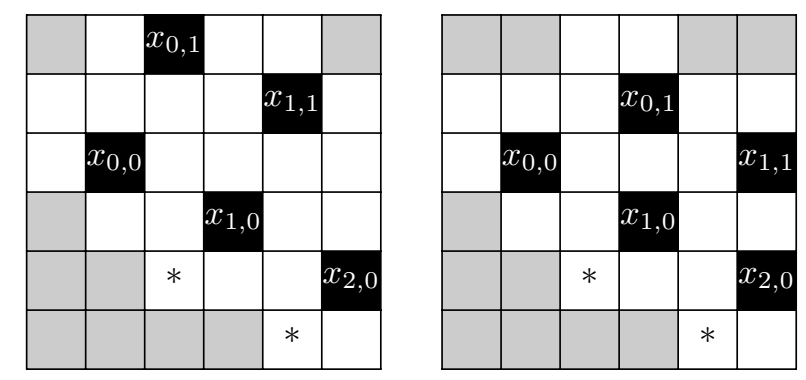

Figure 18: The two possibilities for a set $\sigma$ around a vertex $x:=x_{0,0}$ satisfying (1'). Elements marked with stars are not in $\sigma$ either by assumption or by Lemma 5.7. $x_{1,0}, x_{2,0} \in$ $\sigma$ by Lemma $5.2 ; x_{0,1} \in \sigma$ by the fact that $x_{0,1} \in\left(\mathrm{n}^{3} \mathrm{w}^{3}\right)^{*}(\sigma)$ and $\mathrm{s}^{3} \mathrm{e}^{3}\left(x_{0,1}\right)$ is blocked in $\sigma$ by $x_{2,0} ; x_{1,1} \in \sigma$ again by Lemma 5.2 .

We analyze $\Gamma^{R}$. A set $\sigma$ in $\Gamma$ belongs to this family if and only if every element $x$ in $\left(\mathrm{n}^{3} \mathrm{w}^{3}\right)^{*}(\sigma)$ such that $\mathrm{s}^{3} \mathrm{e}^{3}(x) \notin\left(\mathrm{n}^{3} \mathrm{w}^{3}\right)^{*}(\sigma)$ satisfies either of the following two properties:

$\left(1^{\prime}\right) \mathrm{se}^{2}(x) \in\left(\mathrm{n}^{3} \mathrm{w}^{3}\right)^{*}(\sigma)$ and either $\mathrm{n}^{2} \mathrm{e}(x)$ or $\mathrm{ne}^{2}(x)$ belongs to $\left(\mathrm{n}^{3} \mathrm{w}^{3}\right)^{*}(\sigma)$.

$\left(2^{\prime}\right) \mathbf{s}^{2} \mathrm{e}(x) \in\left(\mathrm{n}^{3} \mathrm{w}^{3}\right)^{*}(\sigma)$ and either $\mathrm{s}^{2} \mathrm{w}(x)$ or $\mathbf{s w}^{2}(x)$ belongs to $\left(\mathrm{n}^{3} \mathrm{w}^{3}\right)^{*}(\sigma)$.

See Figure 18. By Lemma 5.7, $\mathrm{se}^{2}(x)$ and $\mathrm{s}^{2} \mathrm{e}(x)$ cannot both belong to $\left(\mathrm{n}^{3} \mathrm{w}^{3}\right)^{*}(\sigma)$. In particular, (1') and (2') cannot hold simultaneously.

Given a $\langle u, v\rangle$-invariant set $\rho$, we refer to the set of cosets of $\langle u, v\rangle$ contained in $\rho$ as $\rho$ modulo $\langle u, v\rangle$.

Lemma 5.9. $\Gamma^{R}$ is the family of balanced $\langle u, v\rangle$-invariant rhombus tilings modulo $\langle u, v\rangle$ such that the first axis is not equal to $(3,3)$.

Proof. First, suppose that $\sigma$ coincides with a balanced rhombus tiling modulo $\langle u, v\rangle$ in which the first axis is not equal to $(3,3)$. One easily checks that $\sigma=\left(\mathrm{n}^{3} \mathrm{w}^{3}\right)^{*}(\sigma)$, because all elements outside $\sigma$ are blocked; consider Figure 2. Since $(3,3)$ is not the first axis of the tiling, there is an element $x \in \sigma$ such that $\pi(x), \pi^{2}(x) \in \sigma$, where $\pi$ is either $\mathrm{s}^{2} \mathrm{e}$ or $\mathrm{se}^{2}$. Since there are no anti-diagonals $\{q, \mathrm{sw}(q)\}$ in a rhombus tiling, it follows that $\mathrm{s}^{3} \mathrm{e}^{3}(x) \notin \sigma$. As a consequence, $\sigma$ belongs to $\Gamma$. To see that $\sigma$ belongs to $\Gamma^{R}$, apply Proposition 2.1 to deduce that (1') or (2') holds for any $x \in \sigma$.

Next, let $\sigma$ be a set in $\Gamma^{R}$. We want to prove that $\sigma$ forms a rhombus tiling as described in the lemma. Before proceeding, we observe that if $\sigma$ forms a balanced rhombus tiling modulo $\langle u, v\rangle$ in which the first axis is equal to $(3,3)$, then $\sigma$ is a union of $\mathrm{n}^{3} \mathrm{w}^{3}$-cycles and hence in $\Lambda$ rather than in $\Gamma$.

Let $x_{0}$ be an element in $\left(\mathrm{n}^{3} \mathrm{w}^{3}\right)^{*}(\sigma)$ such that $\mathrm{s}^{3} \mathrm{e}^{3}\left(x_{0}\right) \notin\left(\mathrm{n}^{3} \mathrm{w}^{3}\right)^{*}(\sigma)$. Hence $\left(1^{\prime}\right)$ or (2') holds and $x_{0} \in \sigma$. By symmetry, we may assume that (1') holds. As in the previous section, Lemma 5.2 yields that $\mathrm{se}^{2}\left(x_{0}\right)$ and $\mathrm{s}^{2} \mathrm{e}^{4}\left(x_{0}\right)$ both belong to $\sigma$. 
We have that exactly one of $\mathrm{n}^{2} \mathrm{e}\left(x_{0}\right)$ and $n \mathrm{e}^{2}\left(x_{0}\right)$ belongs to $\left(\mathrm{n}^{3} \mathrm{w}^{3}\right)^{*}(\sigma)$, because if both were present, then they would be part of an se-cycle in $\left(\mathrm{n}^{3} \mathrm{w}^{3}\right)^{*}(\sigma)$, which would block $\mathrm{s}^{2} \mathrm{e}^{4}\left(x_{0}\right)$. Let $x_{1}$ be the one of these two elements that belongs to $\left(\mathrm{n}^{3} \mathrm{w}^{3}\right)^{*}(\sigma)$. Since $\mathbf{s}^{3} \mathrm{e}^{3}\left(x_{1}\right) \in\left\{\mathrm{se}^{4}\left(x_{0}\right), \mathrm{s}^{2} \mathrm{e}^{5}\left(x_{0}\right)\right\}$, this element is blocked by $\mathrm{s}^{2} \mathrm{e}^{4}\left(x_{0}\right)$ and hence not in $\left(\mathrm{n}^{3} \mathrm{w}^{3}\right)^{*}(\sigma)$. In particular, $x_{1} \in \sigma$. Moreover, $\mathrm{s}^{2} \mathrm{e}\left(x_{1}\right) \in\left\{\mathrm{e}^{2}\left(x_{0}\right)\right.$, $\left.\mathrm{se}^{3}\left(x_{0}\right)\right\}$, which means that this element is blocked by $\operatorname{se}^{2}\left(x_{0}\right)$ and hence not in $\left(\mathrm{n}^{3} \mathrm{w}^{3}\right)^{*}(\sigma)$ either. As a consequence, $\operatorname{se}^{2}\left(x_{1}\right) \in \sigma$. Since $\sigma$ belongs to $\Gamma^{R}$, as opposed to $\Gamma^{0}$, it follows that $x_{1}$ satisfies $\left(1^{\prime}\right)$.

Proceeding inductively, we obtain a sequence $\left(x_{j}: j \geq 0\right)$ such that each $x_{j}$ satisfies (1') and belongs to $\sigma$ and such that $x_{j+1} \in\left\{\mathrm{ne}^{2}\left(x_{j}\right), \mathrm{n}^{2} \mathrm{e}\left(x_{j}\right)\right\}$. Since $x_{j}=x_{l}$ clearly implies that $x_{j-1}=x_{l-1}$, we obtain that there is a $k$ such that $x_{0}=x_{k}$. In particular, the sequence is periodic and can be extended to all $j \in \mathbb{Z}$ by defining $x_{j}:=x_{j+k}$ for negative $j$.

Now, let $\left(y_{j}: j \in \mathbb{Z}\right)$ be any periodic sequence of elements in $\sigma \backslash(\text { se })^{\infty}(\sigma)$ such that $y_{j+1} \in\left\{\mathrm{ne}^{2}\left(y_{j}\right), \mathrm{n}^{2} \mathrm{e}\left(y_{j}\right)\right\}$ for all $j \in \mathbb{Z}$. By Lemma 5.2, for each $j$, either $\mathrm{s}^{2} \mathrm{e}\left(y_{j}\right)$ or $\mathrm{se}^{2}\left(y_{j}\right)$ belongs to $\sigma$. Suppose that there are distinct indices $i$ and $j$ such that $\operatorname{se}^{2}\left(y_{i}\right), \mathrm{s}^{2} \mathrm{e}\left(y_{j}\right) \in \sigma$. By periodicity, we may assume that $j=i+1$. However,

$$
\mathbf{s}^{2} \mathrm{e}\left(y_{i+1}\right) \in\left\{\mathbf{s}^{2} \mathrm{e}\left(\mathrm{ne}^{2}\left(y_{i}\right)\right), \mathbf{s}^{2} \mathrm{e}\left(\mathrm{n}^{2} \mathrm{e}\left(y_{i}\right)\right)\right\}=\left\{\mathrm{se}^{3}\left(y_{i}\right), \mathrm{e}^{2}\left(y_{i}\right)\right\},
$$

which means that $\mathrm{s}^{2} \mathrm{e}\left(y_{i+1}\right)$ and $\mathrm{se}^{2}\left(y_{i}\right)$ block each other. Hence there is a fixed $\delta \in$ $\left\{\mathrm{se}^{2}, \mathrm{~s}^{2} \mathrm{e}\right\}$ such that $\delta\left(y_{j}\right) \in \sigma$ for all $j \in \mathbb{Z}$. Moreover, with $y_{j}^{\prime}=\delta\left(y_{j}\right)$, we obtain a new periodic sequence $\left(y_{j}^{\prime}: j \in \mathbb{Z}\right)$ of elements in $\sigma \backslash(\text { se })^{\infty}(\sigma)$ such that $y_{j+1}^{\prime} \in$ $\left\{\mathrm{ne}^{2}\left(y_{j}^{\prime}\right), \mathrm{n}^{2} \mathrm{e}\left(y_{j}^{\prime}\right)\right\}$ for all $j \in \mathbb{Z}$.

Write $x_{0, j}:=x_{j}$. For each $j$, let $\epsilon_{j} \in\left\{\mathrm{ne}^{2}, \mathrm{n}^{2} \mathrm{e}\right\}$ be such that $\epsilon_{j}\left(x_{0, j-1}\right)=x_{0, j}$. Induction and the above discussion yield a set $\left\{x_{i, j}: i \geq 0, j \in \mathbb{Z}\right\}$ of elements in $\sigma$ and functions $\delta_{i} \in\left\{\mathrm{se}^{2}, \mathrm{~s}^{2} \mathrm{e}\right\}$ for $i \geq 1$ such that $x_{i, j}=\delta_{i}\left(x_{i-1, j}\right)=\epsilon_{j}\left(x_{i, j-1}\right)$. Clearly, $\left(x_{i, j}: i \geq 0\right)$ is periodic for each $j$, which yields that we may extend our set to all $i$ and hence obtain a set $\left\{x_{i, j}: i, j \in \mathbb{Z}\right\}$. Viewing this set as a subset $\rho$ of $\mathbb{Z}^{2}$ in the natural manner, identifying the coset $x_{0,0}$ with any of its members, we may apply Proposition 2.1 to deduce that $\rho$ is a balanced rhombus tiling. Since each element in $\mathbb{Z}^{2} \backslash \rho$ is adjacent to some element in $\rho$, we conclude that $\sigma$ must coincide with $\rho$ modulo $\langle u, v\rangle$.

\section{Step 7: Proceeding with the family $\Lambda$}

The remaining family is $\Lambda$, which consists of all independent sets $\sigma$ such that $\left(\mathrm{n}^{3} \mathrm{w}^{3}\right)^{*}(\sigma)$ is a union of $\mathrm{n}^{3} \mathrm{w}^{3}$-cycles.

Recall that $d=\operatorname{gcd}\left(u_{1}-u_{2}, v_{1}-v_{2}\right)$. By Lemma 4.4, the number of orbits in $V_{u, v}$ under the permutation se is equal to $d$. Let $\gamma_{0}, \ldots, \gamma_{d-1}$ be these orbits, arranged such that the element $(0, i)$ belongs to $\gamma_{i}$ for $0 \leq i \leq d-1$. Note that $\gamma_{0}, \ldots, \gamma_{d-1}$ are exactly the se-cycles (equivalently, the nw-cycles) in $V_{u, v}$. We identify two cases:

- The exponent $\left|V_{u, v}\right| / d$ of se is not divisible by three.

- The exponent $\left|V_{u, v}\right| / d$ of se is divisible by three. 
We deal with the two cases in Step 8 and Steps 9-12, respectively, the former case being by far the easier.

\section{Step 8: Relating the case $3 \backslash \frac{\left|V_{u, v}\right|}{d}$ to $\Sigma\left(C_{d}\right)$}

First, suppose that the exponent of se is not divisible by three, meaning that $\left|V_{u, v}\right| / d$ is not a multiple of three. In this case, $\gamma_{0}, \ldots, \gamma_{d-1}$ are the $\mathrm{n}^{3} \mathrm{w}^{3}$-cycles in $V_{u, v}$. As a consequence, a set $\sigma$ belongs to $\Lambda$ if and only if $(\mathrm{se})^{*}(\sigma)$ is a union of se-cycles. Moreover, we also have that there are no balanced $\langle u, v\rangle$-invariant rhombus tilings with one axis equal to $(3,3)$; hence $Z\left(\Gamma^{R}\right)=\left|R_{u, v}^{+}\right|-\left|R_{u, v}^{-}\right|$by Lemma 5.9 .

It is clear that two se-cycles $\gamma_{i}$ and $\gamma_{j}$ contain elements blocking each other if and only if $j-i \equiv \pm 1(\bmod d)$. In particular, for a given set $\tau \subseteq \mathbb{Z}_{d}, X_{\tau}:=\bigcup_{i \in \tau} \gamma_{i}$ equals $(\mathrm{se})^{*}(\sigma)$ for some $\sigma \in \Lambda$ if and only if $\tau \in \Sigma\left(C_{d}\right)$, i.e., $\tau$ is an independent set in the cycle graph $C_{d}$. Let $\Lambda(\tau)$ be the family of all $\sigma \in \Lambda$ such that $(\text { se })^{*}(\sigma)=X_{\tau}$.

For $\tau \in \Sigma\left(C_{d}\right)$, write $0 \times \tau:=\{(0, i): i \in \tau\}$. For $\sigma \in \Lambda(\tau) \backslash\{0 \times \tau\}$, let $r:=r_{\sigma}$ be minimal such that $(\sigma \backslash(0 \times \tau)) \cap \gamma_{r}$ is nonempty. It is clear that $\sigma$ stays in $\Sigma\left(X_{\tau}\right)$ and that $r_{\sigma}$ remains the same if $\left(0, r_{\sigma}\right)$ is added to or deleted from $\sigma$. In particular, we obtain a perfect matching on $\Lambda(\tau) \backslash\{0 \times \tau\}$ by pairing $\sigma-\left(0, r_{\sigma}\right)$ with $\sigma+\left(0, r_{\sigma}\right)$.

Taking the union of all these matchings, we obtain a matching on $\Lambda$ such that a set $\sigma$ is unmatched if and only $\sigma=0 \times \tau$ for some $\tau \in \Sigma\left(C_{d}\right)$. By a result due to Kozlov [9, Prop. 5.2], we have the following consequence:

Lemma 5.10. If $\left|V_{u, v}\right| / d$ is not a multiple of three, then $Z(\Lambda)=(-1)^{d} \theta_{d}$.

Recall that $d^{*}=\operatorname{gcd}\left(u_{1}+u_{2}, v_{1}+v_{2}\right)$. Since $d d^{*}$ divides

$$
\operatorname{det}\left(\begin{array}{cc}
u_{1}-u_{2} & v_{1}-v_{2} \\
u_{1}+u_{2} & v_{1}+v_{2}
\end{array}\right)= \pm 2\left|V_{u, v}\right|
$$

we have that $d^{*}$ is not divisible by three. As a consequence, $\theta_{d^{*}}=-1$, which yields that $(-1)^{d} \theta_{d}=-(-1)^{d} \theta_{d} \theta_{d^{*}}$, As a consequence, combining Lemma 5.10 with Lemmas 5.1, 5.8, and 5.9, we obtain Theorem 1.2 in this particular case.

\section{Step 9: Proceeding with the case $3 \mid \frac{\left|V_{u, v}\right|}{d}$}

From now on, assume that the exponent of se is a multiple of three, meaning that three divides $\left|V_{u, v}\right| / d$. For a given set $\sigma \in \Lambda$, write $\hat{\sigma}=\sigma \backslash(\mathrm{se})^{\infty}(\sigma)$. Let $K(\sigma)$ be the set of $\left(\mathrm{ne},\left(\mathrm{n}^{3} \mathrm{w}^{3}\right)^{*}(\hat{\sigma})\right)$-free elements. Hence we first remove all se-cycles and then apply, in order, $\left(\mathrm{n}^{3} \mathrm{w}^{3}\right)^{*}$ and $(\mathrm{ne})^{*}$ to the resulting set. See Figure 19 for an example.

Lemma 5.11. If $\sigma$ belongs to $\Lambda$, then so does $K(\sigma)$. Moreover, (se $)^{\infty}(K(\sigma))=\emptyset$.

Proof. $K(\sigma)$ is obviously a union of $\mathrm{n}^{3} \mathrm{w}^{3}$-cycles, being defined as (ne)* applied to such a union. To settle the first statement in the lemma, it remains to prove that $K(\sigma)$ belongs to $\Delta$. By Lemma 5.2 , it suffices to prove that either $\mathrm{s}^{2} \mathrm{e}(x)$ or $\mathrm{se}^{2}(x)$ belongs to $K(\sigma)$ 


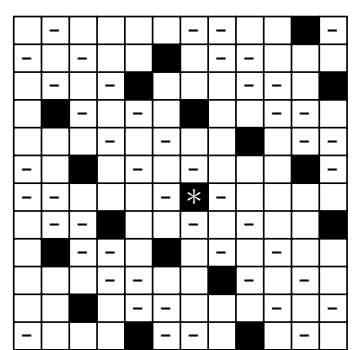

$\sigma$ and $\hat{\sigma}$

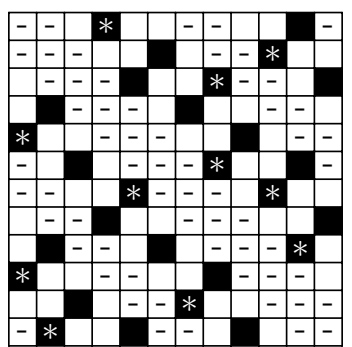

$\sigma^{\prime}:=\left(\mathrm{n}^{3} \mathrm{w}^{3}\right)^{*}(\hat{\sigma})$

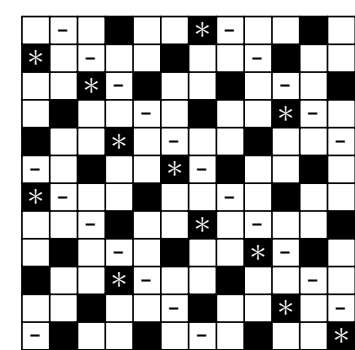

$K(\sigma):=(\mathrm{ne})^{*}\left(\sigma^{\prime}\right)$

Figure 19: On the left a set $\sigma \in \Lambda_{12,12}$ and the subset $\hat{\sigma}$ obtained by removing the element marked with a star; this element belongs to $(\mathrm{se})^{\infty}(\sigma)$. In the middle and on the right, we apply $\left(\mathrm{n}^{3} \mathrm{w}^{3}\right)^{*}$ to $\hat{\sigma}$ and then $(\mathrm{ne})^{*}$ to the result; added elements are marked with stars. se-cycles fully outside the sets are marked with "-".
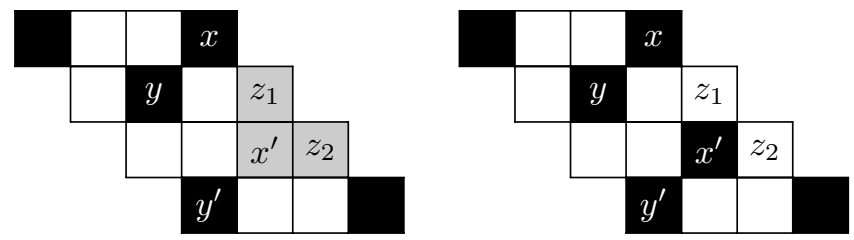

Figure 20: The situation in the proof of Lemma 5.11 around the vertex $x$ in $K(\sigma)$. Given the situation illustrated on the left, we deduce that $z_{1}, z_{2} \notin\left(\mathrm{n}^{3} \mathrm{w}^{3}\right)^{*}(\hat{\sigma})$, which yields the situation illustrated on the right. In particular, $x^{\prime} \in K(\sigma)$.

for each $x \in K(\sigma)$; note that this will imply also the second statement in the lemma. By Lemma 5.5, we are done if $x \in\left(\mathrm{n}^{3} \mathrm{w}^{3}\right)^{*}(\hat{\sigma})$; thus assume that $x \in K(\sigma) \backslash\left(\mathrm{n}^{3} \mathrm{w}^{3}\right)^{*}(\hat{\sigma})$. In particular, $y:=\operatorname{sw}(x) \in K(\sigma)$.

By an induction argument on the number of sw-steps needed to get to an element in $\left(\mathrm{n}^{3} \mathrm{w}^{3}\right)^{*}(\hat{\sigma})$, we may conclude that $y^{\prime}:=\mathrm{s}^{2} \mathrm{e}(y)$ or $x^{\prime}:=\mathrm{se}^{2}(y)$ belongs to $K(\sigma)$; apply Lemma 5.5 in the base case. We want to prove that $x^{\prime}$ belongs to $K(\sigma)$; this will yield the desired result, as $x^{\prime}=\mathrm{s}^{2} \mathrm{e}(x)$. If $y^{\prime} \notin K(\sigma)$, then we are done; thus assume that $y^{\prime} \in K(\sigma)$. This means that the situation is as in the picture on the left in Figure 20 . Since $y^{\prime}=\operatorname{sw}\left(x^{\prime}\right)$, we have that $x^{\prime}$ belongs to $K(\sigma)$ unless it is blocked; apply Lemma 4.2 first to $\sigma^{\prime}:=\left(\mathrm{n}^{3} \mathrm{w}^{3}\right)^{*}(\hat{\sigma})$ and then to $(\mathrm{ne})^{*}\left(\sigma^{\prime}\right)$.

Now, the neighbors of $x^{\prime}$ are $\mathrm{se}(y), \mathrm{s}^{2} \mathrm{e}^{2}(y)$, se $(x)$, and $\mathrm{s}^{2} \mathrm{e}^{2}(x)$. The first two neighbors are blocked by $\mathrm{s}^{2} \mathrm{e}(y)$; hence the remaining neighbors $z_{1}:=\operatorname{se}(x)$ and $z_{2}:=\mathrm{s}^{2} \mathrm{e}^{2}(x)$ have the property that $\operatorname{sw}\left(z_{i}\right) \notin K(\sigma)$. As a consequence, $z_{i}$ belongs to $K(\sigma)$ if and only if $z_{i}$ belongs to $\left(\mathrm{n}^{3} \mathrm{w}^{3}\right)^{*}(\hat{\sigma})$. Yet, applying Lemma 5.5 to $z_{i}$, we then obtain that $\mathrm{s}^{2} \mathrm{e}\left(z_{i}\right)$ or $\mathrm{se}^{2}\left(z_{i}\right)$ belongs to $\left(\mathrm{n}^{3} \mathrm{w}^{3}\right)^{*}(\hat{\sigma})$ and hence to $K(\sigma)$. By Lemma 4.2, this is a contradiction, because both these elements are blocked by $\mathrm{s}^{3} \mathrm{e}^{3}(x)$, which belongs to $K(\sigma)$. It follows that $x^{\prime}$ is not blocked in $K(\sigma)$ and hence belongs to $K(\sigma)$ as desired. 


\section{Step 10: Partitioning $\Lambda$ into subfamilies $\Lambda(K)$}

For each possible $K$, define $\Lambda(K)$ as the family of sets $\sigma$ in $\Lambda(K)$ such that $K(\sigma)=K$. First, note that $\sigma$ belongs to $\Lambda(\emptyset)$ if and only if $(\mathrm{se})^{*}(\sigma)$ is a union of se-cycles. In particular, we may deduce the following, using the same approach as in Step 8:

Lemma 5.12. We have that $Z(\Lambda(\emptyset))=(-1)^{d} \theta_{d}$.

Next, consider a nonempty $K$. Let $\tau_{K}$ be the set of indices $i$ such that $K \cap \gamma_{i}$ is nonempty. We have two cases:

- $\tau_{K}$ is nonempty and not equal to the full set $\mathbb{Z}_{d}$.

- $\tau_{K}$ is equal to $\mathbb{Z}_{d}$.

The remaining case $\tau_{K}=\emptyset$ (i.e., $K=\emptyset$ ) was handled in Lemma 5.12.

\section{Step 11: Relating the case $\tau_{K} \notin\left\{\emptyset, \mathbb{Z}_{d}\right\}$ to a family of rhombus tilings}

For a set $\sigma$ and an index $i$, write $\sigma_{(i)}:=\sigma \cap \gamma_{i}$. We view $i$ as an element in $\mathbb{Z}_{d}$; hence $i$ and $i+d$ are identified. Suppose that $\Lambda(K) \neq \emptyset$ and that $\tau_{K}$ is nonempty and not equal to $\mathbb{Z}_{d}$. Clearly, $d \geq 2$. Moreover, whenever $i \in \tau_{K}$, either $i-1$ or $i+1$ belongs to $\tau_{K}$. Namely, otherwise any given element $x$ in $K_{(i)}$ would not satisfy Lemma 5.2, which would contradict Lemma 5.11. In particular, since $\tau_{K} \neq \mathbb{Z}_{d}$, we cannot have $d=2$. It follows that we may assume that $d>2$.

We claim that for any two consecutive elements $i$ and $i+1$, at least one of them belongs to $\tau_{K}$. Namely, suppose that $i+1 \notin \tau_{K}$ and $i-1 \in \tau_{K}$. Let $x \in K_{(i-1)}$. Then there is no element blocking $x^{\prime}:=\mathrm{se}^{2}(x)$ in $K$; two neighbors of $x^{\prime}$ are in $\gamma_{i+1}$, whereas the other two neighbors are $\mathrm{se}(x)$ and $\mathrm{s}^{2} \mathrm{e}^{2}(x)$, which are not in $K$ by Lemma 5.3 (and Lemma 5.11). Now, suppose that $x^{\prime}$ is not in $K$. Then Lemma 5.2 implies that $\mathrm{s}^{2} \mathrm{e}(x)$ is in $K$. However, applying ne to this element yields $x^{\prime}$, which is a contradiction to $x^{\prime}$ not being (ne, $K$ )-free.

We may write $\tau_{K}$ as a disjoint union of "blocks" of the form $\{i+1, i+2, \ldots, j-1\}$ such that $i, j \notin \tau_{K}$. Let $a_{1}, \ldots, a_{r}$ be the elements outside $\tau_{K}$ in increasing order and let $B_{k}$ be the block between $a_{k}$ and $a_{k+1}$. Hence $B_{k}=\left\{a_{k}+1, \ldots, a_{k+1}-1\right\}$ for $1 \leq k<r$ and $B_{r}=\left\{a_{r}+1, a_{r}+2, \ldots, d+a_{1}-1\right\}$ (computed modulo $\left.d\right)$. For an example, see Figure 19. Assuming that the upper left corner is $(0,0)$, we obtain $r=2, a_{1}=1$, and $a_{2}=7$; hence $b_{1}=\{2,3,4,5,6\}$ and $b_{2}=\{8,9,10,11,0\}$.

Let $\sigma \in \Lambda(K)$. Some element from $\gamma_{a_{i}+1}$ belongs to $\hat{\sigma}$ for each $i$, because there are elements $x$ in $\gamma_{a_{i}+1}$ that are $\left(\right.$ ne, $\left.\left(\mathrm{n}^{3} \mathrm{w}^{3}\right)^{*}(\hat{\sigma})\right)$-free, which is not true for $\operatorname{sw}(x)$.

Lemma 5.13. Let $\Lambda^{R}$ be the union of all families $\Lambda(K)$ such that the corresponding blocks $B_{1}, \ldots, B_{r}$ have size two. Then $\Lambda^{R}$ is the family of balanced $\langle u, v\rangle$-invariant rhombus tilings modulo $\langle u, v\rangle$ such that the first axis is equal to $(3,3)$. 
Proof. Consider a set $K$ as in the lemma. Clearly, $d$ is a multiple of three and $r=d / 3$. Moreover, there is a constant $a \in\{0,1,2\}$ such that $B_{k}=\{3 k-a, 3 k-a+1\}$ and $3 k-a-1,3 k-a+2 \notin \tau_{K}$ for $k \in[r]$. By symmetry, we may assume that $a=2$; hence $B_{k}=\{3 k-2,3 k-1\}$ and $\mathbb{Z}_{d} \backslash \tau_{K}=\{0,3, \ldots, d-3\}$.

Let $x_{0} \in \hat{\sigma}_{(3 k-2)}$. By Lemma 5.2, either $\mathrm{s}^{2} \mathrm{e}\left(x_{0}\right)$ or $\mathrm{se}^{2}\left(x_{0}\right)$ belongs to $\hat{\sigma}$. Since $\mathrm{s}^{2} \mathrm{e}\left(x_{0}\right) \in \gamma_{3 k-3}$, we must have that $x_{1}:=\mathrm{se}^{2}\left(x_{0}\right)$ belongs to $\hat{\sigma}$. Using the same argument and the fact that $3 k \notin \tau_{K}$, we obtain that $x_{2}:=\mathrm{s}^{2} \mathrm{e}\left(x_{1}\right)=\mathrm{s}^{3} \mathrm{e}^{3}\left(x_{0}\right)$ belongs to $\hat{\sigma}$. Proceeding inductively, we may deduce that the entire $\mathrm{n}^{3} \mathrm{w}^{3}$-cycles containing $x_{0}$ and $x_{1}$ are included in $\hat{\sigma}$. This is true for every choice of $k$. Moreover, there are no other elements in $\hat{\sigma}_{(3 k-2)}$ or $\hat{\sigma}_{(3 k-1)}$, as these are all blocked by the $\mathrm{n}^{3} \mathrm{w}^{3}$-cycles containing $x_{0}$ and $x_{1}$. In particular, $\sigma=\hat{\sigma}=K(\sigma)$.

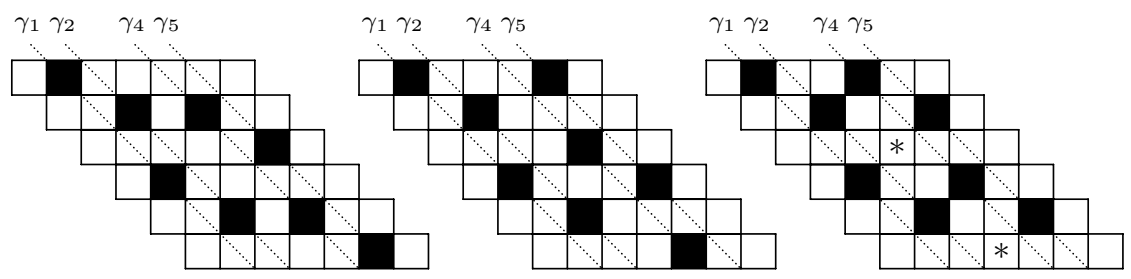

Figure 21: The three possible ways the two sets $\sigma_{(1)} \cup \sigma_{(2)}$ and $\sigma_{(4)} \cup \sigma_{(5)}$ can be related. In the third case, the elements marked with stars are not blocked and hence belong to $K(\sigma)$.

It remains to prove that the condition in Proposition 2.1 is satisfied for every element in $\sigma$. The condition holding for the south-east and the north-west directions is an immediate consequence of the above discussion. For the other two directions, note that the two sets $\sigma_{(3 k-2)} \cup \sigma_{(3 k-1)}$ and $\sigma_{(3 k+1)} \cup \sigma_{(3 k+2)}$ may relate to each other in three different ways; see Figure 21 (this is the case $k=1$ ). However, in the rightmost case in the figure, we have that the elements marked with stars belong to $K(\sigma)$, as they are not blocked. This is a contradiction to the fact that $K_{(3 k)}$ is empty. It follows that only the two leftmost cases are possible. Inspecting these cases, one easily checks that the condition in the proposition holds for the south-west and the north-east directions.

Conversely, one easily checks that a rhombus tiling modulo $\langle u, v\rangle$ as in the lemma belongs to $\Lambda^{R}$.

Lemma 5.14. If some block $B_{i}$ has size at least three, then $Z(\Lambda(K))=0$.

Proof. Let $B_{i}$ be a block of size at least three; we may assume that $k=1$ and $B_{1}=$ $\{1, \ldots, t\}$ for some $t \geq 3$. We divide the proof into steps:

\section{Step I}

First, let us get rid of all $\sigma$ in $\Lambda(K)$ such that $\sigma_{(1)}$ is not a full $\mathrm{n}^{3} \mathrm{w}^{3}$-cycle; the procedure is illustrated in Figure 22. Let $\Lambda^{1}(K)$ be the family of such $\sigma$. For $\sigma \in \Lambda^{1}(K)$, let $x_{1}:=$ $x_{1}(\sigma) \in \sigma_{(1)}$ be minimal (with respect to some total order) such that $x_{3}:=\mathrm{n}^{3} \mathrm{w}^{3}\left(x_{1}\right) \notin \sigma_{(1)}$. 


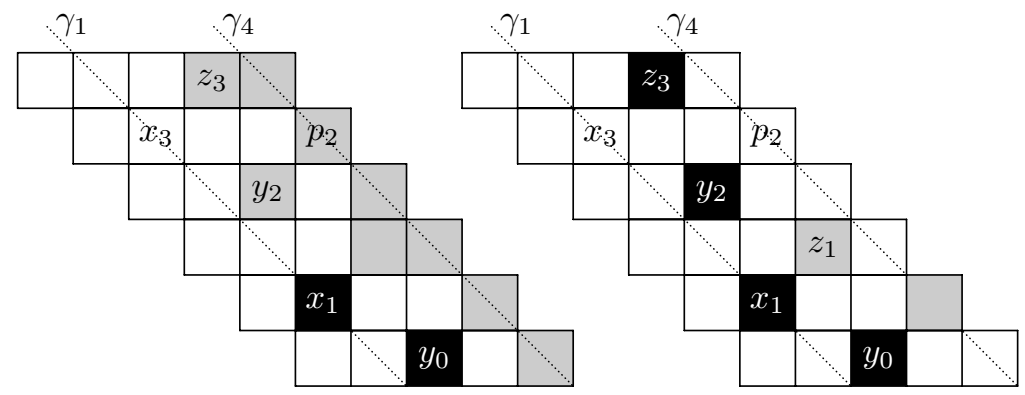

Figure 22: The situation in Step I of the proof of Lemma 5.14. As illustrated in the picture on the left, we can match with the element $y_{2}$ whenever $z_{3} \notin \sigma$ or $p_{2} \in \sigma$. For the remaining case illustrated on the right, we can match with the element $z_{1}$.

Since $\sigma_{(0)}$ is empty, Lemma 5.2 yields that $y_{0}:=\mathrm{se}^{2}\left(x_{1}\right)$ belongs to $\sigma_{(2)}$. Define a matching on $\Lambda^{1}(K)$ by pairing with the element $y_{2}:=\mathrm{n}^{3} \mathrm{w}^{3}\left(y_{0}\right)$ whenever possible; matched pairs have the form $\left\{\sigma-y_{2}, \sigma+y_{2}\right\}$. Note that $y_{2} \in \gamma_{2}$. In particular, the definition of $x_{1}(\sigma)$ does not depend on whether $y_{2}$ is present or not.

We may always add $y_{2}$ to $\sigma$ without ending up outside $\Lambda^{1}(K)$, because $x_{1}$ assures that $y_{2}$ satisfies Lemma 5.2. Moreover, $y_{2}$ is unblocked in $\sigma$, as it belongs to the same $\mathrm{n}^{3} \mathrm{w}^{3}$-cycle in $K_{(2)}$ as $y_{0}$. Hence problems may occur only if we remove $y_{2}$ from $\sigma$. Indeed, we end up outside $\Lambda^{1}(K)$ if and only if $z_{3}:=\mathrm{n}^{2} \mathrm{w}\left(y_{2}\right) \in \sigma_{(3)}$ and $p_{2}:=\mathrm{ne}\left(y_{2}\right) \notin \sigma_{(4)}$. Namely, this is the one situation in which $z_{3}$ does not satisfy Lemma 5.2 for $\sigma-y_{2}$.

Now, let $\hat{\Lambda}^{1}(K)$ be the family of unmatched sets in $\Lambda^{1}(K)$. With notation as above for a given set $\sigma \in \hat{\Lambda}^{1}(K)$, consider the element $z_{1}:=\mathrm{s}^{3} \mathrm{e}^{3}\left(z_{3}\right) ; z_{1}$ belongs to the same $\mathrm{n}^{3} \mathrm{w}^{3}$-cycle in $K_{(3)}$ as $z_{3}$ and is hence unblocked in $\sigma$. We may add or delete $z_{1}$ and still have a set in $\hat{\Lambda}^{1}(K)$. Namely, $z_{1}$ satisfies Lemma 5.2, thanks to $y_{0}$. Moreover, the removal of $z_{1}$ may alter the status with respect to Lemma 5.2 of no other elements but $y_{2}$ and $p_{2}$, and $y_{2}$ already satisfies this lemma thanks to $x_{1}$, whereas $p_{2}$ is not even present in $\sigma$. Hence we obtain a perfect matching on $\hat{\Lambda}^{1}(K)$ by pairing $\sigma-z_{1}$ with $\sigma+z_{1}$.

\section{Step II}

Let $\Lambda^{2}(K)$ be the remaining family; thus $\Lambda^{2}(K)=\Lambda(K) \backslash \Lambda^{1}(K)$. Note that $\sigma_{(1)}$ is an $\mathrm{n}^{3} \mathrm{w}^{3}$-cycle whenever $\sigma \in \Lambda^{2}(K)$. Applying Lemma 5.2 to each element in $\sigma_{(1)}$, we may deduce that $\sigma_{(2)}$ also has this property. There are now two cases for $\sigma \in \Lambda^{2}(K)$ :

- $\gamma_{4}$ is contained in $(\mathbf{s e})^{\infty}(\sigma)$; hence $\gamma_{4}$ forms a full se-cycle in $(\mathbf{s e})^{*}(\sigma)$. Let $\Lambda^{2,1}(K)$ be the family of such sets $\sigma$.

- $\gamma_{4}$ is not contained in $(\mathbf{s e})^{\infty}(\sigma)$. Let $\Lambda^{2,2}(K)$ be the family of such sets $\sigma$. 


\section{Step II.a}

Define a matching on $\Lambda^{2,1}(K)$ by pairing with the element $(0,4)$ whenever possible. A set $\sigma$ remains unmatched if and only if $\sigma_{(4)}=\{(0,4)\}$. We will get rid of the family $\Lambda^{4}(K)$ of such sets in Step III below.

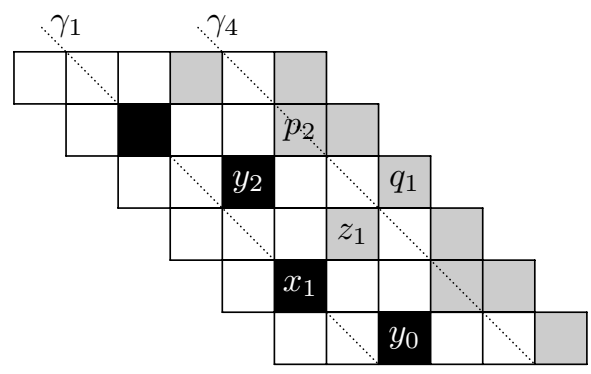

Figure 23: The situation in Step II.b of the proof of Lemma 5.14. We can match with the element $z_{1}$ whenever $p_{2} \notin \sigma$ or $q_{1} \in \sigma$. Compare to the situation in Step I; see Figure 22 .

\section{Step II.b}

For $\sigma \in \Lambda^{2,2}(K)$, suppose that there is an element $y_{2}$ in $\sigma_{(2)}$ such that either $p_{2}:=\operatorname{ne}\left(y_{2}\right)$ does not belong to $\sigma_{(4)}$ or $q_{1}:=\mathrm{e}^{3}\left(y_{2}\right)$ does belong to $\sigma_{(5)}$. We assume that we have chosen $y_{2}:=y_{2}(\sigma)$ to be minimal with this property (with respect to some total order of $\left.\sigma_{(2)}\right)$.

We claim that we may add or delete $z_{1}:=\operatorname{se}^{2}\left(y_{2}\right)$ without ending up outside $\Lambda^{2,2}(K)$ and without altering the value of $y_{2}(\sigma)$. For the first part of the claim, Lemma 5.2 is satisfied for $z_{1}$ thanks to $y_{0}$ and for $p_{2}$ - if present - thanks to $q_{1}$. Moreover, $z_{1}$ is unblocked in $\sigma$ by assumption and belongs to $K\left(\sigma-z_{1}\right)$ thanks to $x_{1}:=\mathbf{s}^{2} \mathrm{e}\left(y_{2}\right)=\operatorname{sw}\left(z_{1}\right)$. For the second part of the claim, just note that $z_{1}$ belongs to $\gamma_{3}$ and that no elements from this set are used in the definition of $y_{2}(\sigma)$. See Figure 23 for an illustration.

As a consequence, we obtain a matching on $\Lambda^{2,2}(K)$ by pairing with the element $y_{2}(\sigma)$.

\section{Step III}

The family $\Lambda^{3}(K)$ of unmatched elements in $\Lambda^{2,2}(K)$ consists of all $\sigma$ such that, for every $y_{2}$ in $\sigma_{(2)}, p_{2}:=\operatorname{ne}\left(y_{2}\right)$ belongs to $\sigma_{(4)}$ and $q_{1}:=\mathrm{e}^{3}\left(y_{2}\right)$ does not belong to $\sigma_{(5)}$. It follows by Lemma 5.2 applied to $p_{1}$ that $z_{1}:=\mathrm{se}^{2}\left(y_{2}\right)$ belongs to $\sigma_{(3)}$. As a consequence, $\sigma_{(i)}$ forms a full $\mathrm{n}^{3} \mathrm{w}^{3}$-cycle for $i=1,2,3,4$, whereas $\sigma_{(5)}$ is empty. Moreover, $\sigma_{(i)}$ is uniquely determined by $\sigma_{(1)}$ for $i=2,3,4$.

To conclude the proof, it suffices to prove that $Z\left(\Lambda^{3}(K)\right)+Z\left(\Lambda^{4}(K)\right)=0$, where $\Lambda^{4}(K)$ is the family of unmatched sets from Step II.b. Now, we obtain a bijection $\varphi$ from $\Lambda^{3}(K)$ to $\Lambda^{4}(K)$ by removing $\sigma_{(3)}$ and $\sigma_{(4)}$ - an even number of elements - and adding in the single element $(0,4)$. Namely, $\varphi$ is certainly well-defined and surjective, and injectivity follows from the observation just made about $\sigma_{(i)}$ being uniquely determined by $\sigma_{(1)}$. See 

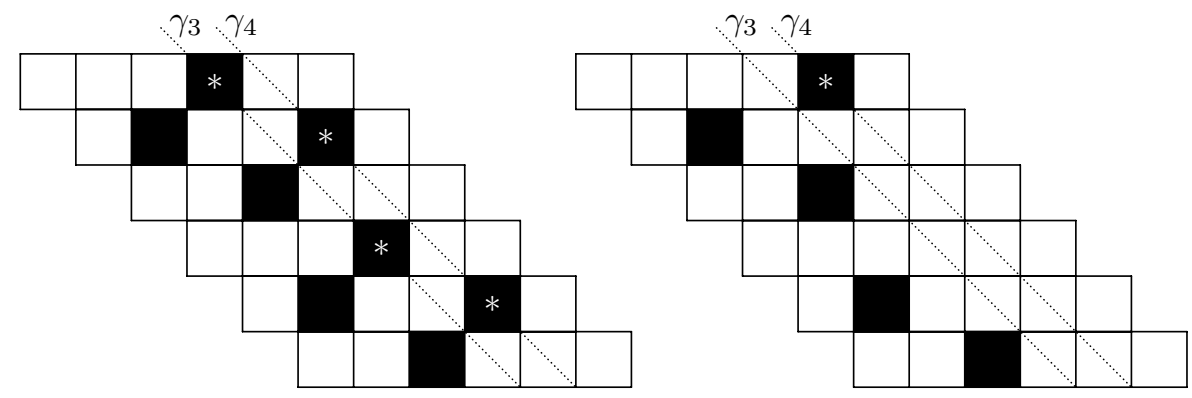

$$
\sigma \in \Lambda_{u, v}^{3}(K) \quad \varphi(\sigma) \in \Lambda_{u, v}^{4}(K)
$$

Figure 24: The function $\varphi$ applied to a set $\sigma$ in $\Lambda^{3}(K)$ in Step III of the proof of Lemma 5.14; the elements marked with stars in the left-hand picture are replaced with the single star-marked element in the right-hand picture.

Figure 24 for an illustration. Since the parity of the size of each set alters under $\varphi$, we obtain that $Z\left(\Lambda^{3}(K)\right)=-Z\left(\Lambda^{4}(K)\right)$. As a consequence, we are done.

\section{Step 12: Relating the case $\tau_{K}=\mathbb{Z}_{d}$ to $\Lambda(\emptyset)$}

The very final step is that $\tau_{K}$ equals the full set $\mathbb{Z}_{d}$. This means that $K_{(i)}$ forms an $\mathrm{n}^{3} \mathrm{w}^{3}$-cycle for each $i$. There is a set $K$ with this property such that $\Lambda(K) \neq \emptyset$ if and only if $d^{*}:=\operatorname{gcd}\left(u_{1}+u_{2}, v_{1}+v_{2}\right)$ is a multiple of three. Namely, $K_{(i)}$ and $K_{(i+1)}$ do not block if and only if $K_{(i+1)}=\mathrm{se}^{2}\left(K_{(i)}\right)=\mathrm{n}^{2} \mathrm{w}\left(K_{(i)}\right)$. Since this must hold for all $i$, we have that $K$ exists if and only if the lattice $\mathbb{I}_{3}$ generated by $(1,2)$ and $(-2,-1)$ contains $\langle u, v\rangle$. This is easily seen to be equivalent to $u_{1}+u_{2}$ and $v_{1}+v_{2}$ both being divisible by three. $K$ is then equal to either of the three cosets of $\hat{\mathbb{I}}_{3}:=\mathbb{I}_{3} /\langle u, v\rangle$, i.e.,

$$
K=\hat{\mathbb{I}}_{3}^{(q)}:=\mathrm{e}^{q}\left(\hat{\mathbb{I}}_{3}\right)=\left\{(i, j) \in V_{u, v}:(i+j) \bmod 3=q\right\},
$$

where $q \in\{0,1,2\}$.

Recalling the discussion from the very beginning of Section 5, we note that there is no loss of generality assuming that $d$ is divisible by three. Otherwise, just start from the beginning, replacing $u_{2}$ and $v_{2}$ with $-u_{2}$ and $-v_{2}$, respectively, thereby swapping the values of $d$ and $d^{*}$.

Remark. Before proceeding, note that if $d^{*}$ is not a multiple of three, then we are done. Namely, collecting values from Lemmas $5.1,5.8,5.9,5.12,5.13$, and 5.14 , we obtain Theorem 1.2; in Lemma 5.12, note that $(-1)^{d} \theta_{d}=-(-1)^{d} \theta_{d} \theta_{d^{*}}$, as $d^{*}$ is not a multiple of three.

Lemma 5.15. Suppose that $d$ and $d^{*}$ are both multiples of three. Then

$$
Z\left(\Lambda\left(\hat{\mathbb{I}}_{3}^{(q)}\right)\right)=-Z(\Lambda(\emptyset))
$$


for $q \in\{0,1,2\}$. As a consequence, $Z(\Lambda)$ equals $-2(-1)^{d} \theta_{d}=-(-1)^{d} \theta_{d} \theta_{d^{*}}$ plus the number of balanced $\langle u, v\rangle$-invariant rhombus tilings modulo $\langle u, v\rangle$ such that the first axis is equal to $(3,3)$.

Remark. This implies Theorem 1.2; collect values from this lemma and Lemmas 5.1, 5.8, and 5.9.

Proof. The latter statement in the lemma follows from the former. To see this, apply Lemmas 5.12, 5.13, and 5.14 and use the fact that there are three choices for $q$. The contribution of the rhombus tilings in Lemma 5.13 being positive is because a rhombus tiling with one axis equal to $(3,3)$ contains an even number of cosets of $\langle u, v\rangle$.

We want to prove that the partition function of the union $\Omega:=\Lambda\left(\hat{\mathbb{I}}_{3}^{(q)}\right) \cup \Lambda(\emptyset)$ vanishes. By symmetry, we may assume that $q=0$.

Let $\Omega^{\prime}$ be the subfamily of $\Omega$ consisting of all $\sigma$ with the property that there is an index $i$ such that $\sigma_{(i)} \cap(\mathrm{se})^{\infty}(\sigma)$ contains an element $x$ different from $(0, i)$. We obtain a perfect matching on $\Omega^{\prime}$ by pairing with $(0, i)$ for the smallest index $i$ with the given property.

Write $c_{(i)}:=\hat{\mathbb{I}}_{3} \cap \gamma_{i}$; it is clear that $c_{(i)}$ is an $\mathrm{n}^{3} \mathrm{w}^{3}$-cycle. We divide the remaining family $\Omega_{1}:=\Omega \backslash \Omega^{\prime}$ into subfamilies in the following manner: For any $\sigma \in \Omega_{1}$, let $\mathrm{a}(\sigma):=\left(a_{i}(\sigma): i \in[0, d / 3-1]\right)$ be the sequence defined as follows:

$$
a_{i}(\sigma)= \begin{cases}\infty & \text { if } \sigma_{(3 i)}=\{(0,3 i)\} \text { and }(0,3 i) \in(\mathrm{se})^{\infty}(\sigma) ; \\ 1 & \text { if } \sigma_{(3 i)}=c_{(3 i)} ; \\ 0 & \text { if } \sigma_{(3 i)}=\emptyset ; \\ -1 & \text { otherwise. }\end{cases}
$$

For a vector $\mathrm{a} \in\{-1,0,1, \infty\}^{d / 3}$, let $\Omega_{1}(\mathrm{a})$ be the subfamily of $\Omega_{1}$ consisting of all $\sigma$ such that $\mathrm{a}=\mathrm{a}(\sigma)$. Our goal is to prove that

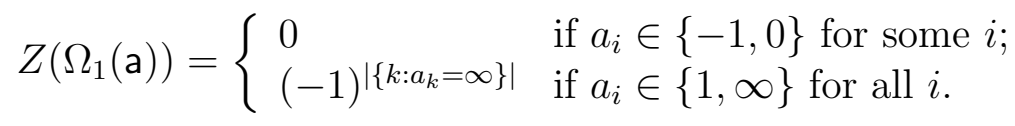

Summing over all a, we will obtain the desired result.

We divide into a number of cases:

(A) $a_{i}=-1$ for some $i$. Let $i$ be maximal with this property. Let $\sigma \in \Omega_{1}(\mathrm{a})$. By construction, there exists an element $x$ in $\sigma_{(3 i)} \backslash(\mathrm{se})^{\infty}(\sigma)$ such that $\mathrm{n}^{3} \mathrm{w}^{3}(x) \notin \sigma$. Let $x:=x(\sigma)$ be maximal with this property (with respect to some total order). Let $\Omega_{1, x}(\mathrm{a})$ be the subfamily of $\Omega_{1}(\mathrm{a})$ consisting of all $\sigma$ satisfying $x(\sigma)=x$.

Consider a set $\sigma$ in $\Omega_{1, x}(\mathrm{a})$. By Lemma 5.2, at least one of the two elements $\operatorname{se}^{2}(x)$ and $\mathrm{s}^{2} \mathrm{e}(x)$ belongs to $\sigma$. Let $\Omega_{1, x, \mathrm{e}}(\mathrm{a})$ be the subfamily consisting of those $\sigma$ that satisfy $\operatorname{se}^{2}(x) \in \sigma$ and write $\Omega_{1, x, \mathrm{~s}}(\mathrm{a}):=\Omega_{1, x}(\mathrm{a}) \backslash \Omega_{1, x, \mathrm{e}}(\mathrm{a})$.

Writing $y_{0}:=\mathrm{se}^{2}(x)$ and $x_{1}:=x$, the situation for a set $\sigma$ in $\Omega_{1, x, \mathrm{e}}(\mathrm{a})$ is almost identical to that in Step I of the proof of Lemma 5.14; see Figure 22. The one 
difference is that $\sigma_{(3 i-1)}$ is not necessarily empty, whereas the corresponding set $\sigma_{(0)}$ in the proof of Lemma 5.14 is empty. However, $\sigma_{(0)}$ being empty is used in that proof only to conclude that $y_{0} \in \sigma$, which we already know in the present case.

Applying the procedure in Step I of the proof of Lemma 5.14, we obtain a perfect matching on $\Omega_{1, x, \mathbf{e}}(\mathrm{a})$. Namely, the elements $y_{2}$ and $z_{1}$ used in the matching belong to $\gamma_{3 i+1}$ and $\gamma_{3 i+2}$, respectively, and hence do not have any impact on $x(\sigma)$ or a $(\sigma)$. Moreover, $y_{2}$ is different from $y_{0}$, as otherwise $x=\mathrm{n}^{3} \mathrm{w}^{3}(x)$, which is impossible. Hence $y_{2}$ and $z_{1}$ have no impact on whether $\sigma$ belongs to $\Omega_{1, x, \mathrm{e}}(\mathrm{a})$ or $\Omega_{1, x, \mathbf{s}}(\mathrm{a})$.

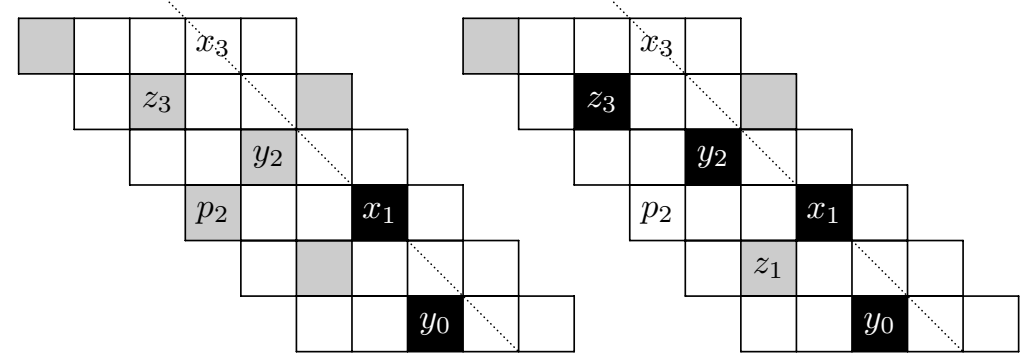

Figure 25: The situation for a set $\sigma$ in $\Omega_{1, x, \mathbf{s}}(\mathrm{a})$ in case (A) of the proof of Lemma 5.15; $x_{1}=x$. The reflection about the dotted line yields the situation in Step I of the proof of Lemma 5.14; compare to Figure 22.

For the family $\Omega_{1, x, \mathbf{s}}(\mathrm{a})$, redefine $y_{0}$ as $\mathbf{s}^{2} \mathrm{e}(x)$. Since $\mathrm{se}^{2}(x) \notin \sigma$, we must have that $y_{0} \in \sigma$. Now, taking the reflection of the given configuration about the line through $x_{1}:=x$ with slope in direction se, we again obtain the situation in Step I of the proof of Lemma 5.14; see Figure 25. Proceeding as in that proof, we obtain a perfect matching on $\Omega_{1, x, \mathbf{s}}(\mathrm{a})$. Here, one should note that $z_{1}:=\mathrm{sw}(x)$ does have an impact on whether $\sigma$ belongs to $\Omega_{1, x, \mathrm{~s}}(\mathrm{a})$ or $\Omega_{1, x, \mathrm{e}}(\mathrm{a})$ if $z_{1}=\mathrm{se}^{2}(x)$, but if this is true, then $p_{2}=x \in \sigma$, where $p_{2}$ is defined as in Figure 25. In particular, the situation in the picture on the right in the figure never occurs, meaning that we never match with $z_{1}$.

(B) $a_{j} \neq-1$ for each $j$ and $a_{i}=0$ for some $i$. Let $i$ be maximal with this property. We identify three different subcases:

- $a_{i-1}=\infty$. In this case, all $\sigma$ in $\Omega_{1}(\mathrm{a})$ have the property that $\sigma_{(3 i-2)}$ and $\sigma_{(3 i)}$ are empty. As a consequence, the only possibilities for $\sigma_{(3 i-1)}$ are $\emptyset$ and $\{(0,3 i-1)\}$. In terms of partition functions, these two possibilities cancel out; thus $Z\left(\Omega_{1}(\mathrm{a})\right)=0$.

- $a_{i-1}=0$. Then the possibilities for $\sigma_{(3 i-2)} \cup \sigma_{(3 i-1)}$ are $\emptyset, c_{(3 i-2)} \cup c_{(3 i-1)}$, $\{(0,3 i-2)\}$, and $\{(0,3 i-1)\}$. Since $\left|\sigma_{(3 i-2)} \cup \sigma_{(3 i-1)}\right|$ is even in two cases and odd in two cases, it follows that $Z\left(\Omega_{1}(\mathrm{a})\right)=0$.

- $a_{i-1}=1$. First, we note that there exists a perfect matching on the subfamily of $\Omega_{1}(\mathrm{a})$ consisting of those $\sigma$ with the property that $\sigma_{(3 i-2)}$ is nonempty. Namely, 


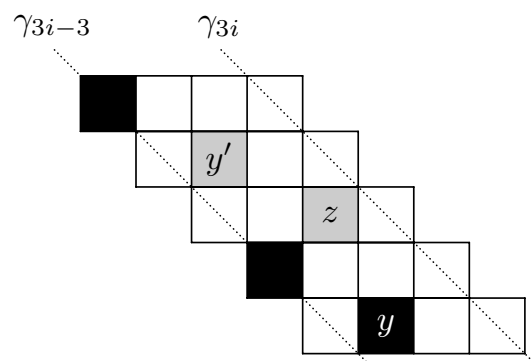

Figure 26: The case that $a_{i}=0, a_{i-1}=1$, and $y \in \sigma_{(3 i-2)}$ in case (B) of the proof of Lemma 5.15.

if $y \in \sigma_{(3 i-2)}$, then we may add or delete $z:=\mathrm{n}^{2} \mathrm{w}(y) \in \gamma_{3 i-1}$ without ending up outside $\Omega_{1}(\mathrm{a})$. To see this, note that $\mathrm{n}^{2} \mathrm{w}(z)$ belongs to $\gamma_{3 i}$ and is hence not in $\sigma$, whereas $y^{\prime}:=\mathrm{nw}^{2}(z)$ already satisfies Lemma 5.2 thanks to the fact that $\mathrm{s}^{2} \mathrm{e}\left(y^{\prime}\right) \in c_{(3 i-3)}=\sigma_{(3 i-3)}$. See Figure 26 for an illustration.

What remains is the subfamily consisting of all $\sigma$ such that $\sigma_{(3 i-2)}$ is empty. The situation then coincides with that for the case $a_{i-1}=\infty$ above, meaning that $\emptyset$ and $\{(0,3 i-1)\}$ are the two possibilities for $\sigma_{(3 i-1)}$. Again, the partition function vanishes.

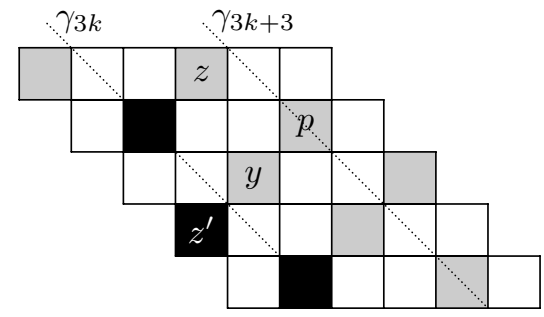

Figure 27: A set $\sigma$ in $\Omega_{1, k}(\mathrm{a})$ in case $(\mathrm{C})$ of the proof of Lemma 5.15. We have that $z \notin \sigma$ or $p \in \sigma$.

(C) $a_{i} \in\{1, \infty\}$ for each $i$. For $k$ in $[0, d / 3-1]$, let $\Omega_{1, k}($ a $)$ be the subfamily of $\Omega_{1}(\mathrm{a})$ consisting of all $\sigma$ such that $\sigma_{(3 k-1)}$ is nonempty and such that $\sigma_{(3 i-1)}$ is empty for all $i \in[0, k-1]$. Note that $\sigma_{(3 k)}=c_{(3 k)}$, as $\sigma_{(3 k-1)}$ is nonempty.

Let $z^{\prime}$ be maximal in $\sigma_{(3 k-1)}$ with respect to some total order. We claim that we may add or delete $y:=$ ne $\left(z^{\prime}\right) \in \sigma_{(3 k+1)}$ without ending up outside $\Omega_{1, k}($ a) or affecting the maximality of $z^{\prime}$; see Figure 27. The latter is obvious. To prove the former, note that $\mathrm{s}^{2} \mathrm{e}(y) \in \sigma_{(3 k)}$ and that $z:=\mathrm{n}^{2} \mathrm{w}(y)$ cannot belong to $\sigma_{(3 k+2)}$ unless $a_{k+1}=1$ and hence $p:=\operatorname{se}^{2}(z) \in \sigma_{(3 k+3)}$; thus Lemma 5.2 is satisfied both in $\sigma-y$ and in $\sigma+y$. As a consequence, matching with $y$ yields a perfect matching on $\Omega_{1, k}(\mathrm{a})$.

The remaining family $\Omega_{2}(\mathrm{a})$ consists of all $\sigma$ in $\Omega_{1}(\mathrm{a})$ such that $\sigma_{(3 k-1)}$ is empty for all $k$. This implies that $\sigma_{(3 k+1)}=c_{(3 k+1)}$ if $\sigma_{(3 k)}=c_{(3 k)}$ (equivalently, $a_{k}=1$ ) and 
$\sigma_{(3 k+1)}=\emptyset$ if $\sigma_{(3 k)}=(0,3 k)$ (equivalently, $\left.a_{k}=\infty\right)$. It follows that $\Omega_{2}(\mathrm{a})$ consists of the single element

$$
\sigma_{\mathrm{a}}:=\left\{(0,3 k): a_{k}=\infty\right\} \cup \bigcup_{k: a_{k}=1}\left(c_{(3 k)} \cup c_{(3 k+1)}\right)
$$

Since $c_{(3 k)}$ and $c_{(3 k+1)}$ have the same number of elements, we may conclude that $Z\left(\Omega_{1}(\mathrm{a})\right)=Z\left(\Omega_{2}(\mathrm{a})\right)=(-1)^{\left|\left\{k: a_{k}=\infty\right\}\right|}$.

\section{Concluding remarks}

Our main discovery is an enumerative connection between the simplicial complex $\Sigma_{u, v}$ and the family $R_{u, v}$ of balanced $\langle u, v\rangle$-invariant rhombus tilings. Unfortunately, our proof is quite technical in nature, ranging over several steps, and does not reveal much of the inner structure of $\Sigma_{u, v}$. In particular, the following question remains unanswered:

- Is there a way to "understand" the connection between $\Sigma_{u, v}$ and $R_{u, v}$ ?

We would be particularly interested in hearing about interpretations of this connection in the language of statistical mechanics.

One may also consider other graphs with a grid structure. Fendley and Schoutens [3] examined several such graphs and obtained exact results for octagon-square and enneagontriangle grids. In fact, they managed to compute the (co-)homology of the relevant independence complexes.

Another important example, closely related to the square grid $S_{m, n}$, is the "cylinder" $C_{m, n}=I_{m} \times C_{n}$, where $I_{m}$ is the graph obtained from $C_{m}$ by removing the edge between 0 and $m-1$. Hence we obtain $C_{m, n}$ from $S_{m, n}$ by removing the edge between $(0, j)$ and $(m-1, j)$ for every $j \in \mathbb{Z}_{n}$. As Fendley et al. observed [4], the independence complex of $C_{m, n}$ appears to have nice enumerative properties. Specifically, for $m \leq 11$, all roots of the transfer matrix associated to $\left\{C_{m, n}: n \geq 1\right\}$ are roots of unity; see Table 1 . It seems reasonable to conjecture that this is true in general. Moreover, the situation for $m \leq 11$ suggests that $Z\left(C_{m, n}\right)=1$ for odd $n$ unless $\operatorname{gcd}(m-1, n)$ is a multiple of three, in which case $Z\left(C_{m, n}\right)=-2$; see Table 2 . For even $n$, the situation appears to be far more complicated; see Table 3 for the value of $Z\left(C_{m, n}\right)$ for small $m$ and small even $n$. So far, we have been unsuccessful in our attempts to adapt our methods to $C_{m, n}$.

For the triangular grid, numerical computations [4] suggest that the partition function (evaluated at $z=-1$ ) grows approximately as $1.14^{N}$, where $N$ is the number of vertices modulo the given periodicity. As a comparison, our results imply that $\left|Z\left(\Sigma_{m, n} ;-1\right)\right| \leq$ $D_{m}$, where $D_{m} \sim\left(\frac{1+\sqrt{5}}{2}\right)^{m} \approx 1.618^{m}$, which is subexponential in the vertex size $m n$. Hence the behavior of the triangular grid appears to be completely different from that of the square grid. In this context, it is worth mentioning that the case $z=+1$ is well-understood for the triangular grid, thanks to a beautiful result due to Baxter [1]. 
Table 1: The characteristic polynomial $Q_{m}(t)$ of the transfer matrix associated to the family $\left\{Z\left(C_{m, n}\right): n \geq 1\right\}$ for small $m$. We multiply by $(t+1) \cdot\left(t^{4}+(-1)^{m}\right)$ for optimal display.

\begin{tabular}{|l|l|}
\hline$m$ & $(t+1) \cdot\left(t^{4}+(-1)^{m}\right) \cdot Q_{m}(t)$ \\
\hline \hline 1 & $\left(t^{4}-1\right)\left(t^{3}+1\right)$ \\
\hline 2 & $\left(t^{8}-1\right)$ \\
\hline 3 & $\left(t^{8}-1\right)\left(t^{2}-1\right)$ \\
\hline 4 & $\left(t^{8}-1\right)\left(t^{3}+1\right)\left(t^{2}-1\right)$ \\
\hline 5 & $\left(t^{10}-1\right)\left(t^{8}-1\right)$ \\
\hline 6 & $\left(t^{14}-1\right)\left(t^{8}-1\right)\left(t^{4}-1\right)$ \\
\hline 7 & $\left(t^{18}-1\right)\left(t^{12}-1\right)\left(t^{4}+1\right)\left(t^{3}+1\right)\left(t^{2}-1\right)$ \\
\hline 8 & $\left(t^{22}-1\right)\left(t^{16}-1\right)^{2}\left(t^{4}-1\right)\left(t^{2}-1\right)$ \\
\hline 9 & $\left(t^{26}-1\right)\left(t^{20}-1\right)^{2}\left(t^{14}-1\right)\left(t^{10}+1\right)\left(t^{4}+1\right)$ \\
\hline 10 & $\left(t^{30}-1\right)\left(t^{24}-1\right)^{3}\left(t^{18}-1\right)^{2}\left(t^{4}-1\right)^{2}\left(t^{3}+1\right)$ \\
\hline 11 & $\left(t^{34}-1\right)\left(t^{28}-1\right)^{3}\left(t^{22}-1\right)^{4}\left(t^{14}-1\right)\left(t^{8}+1\right)\left(t^{4}+1\right)^{2}\left(t^{2}-1\right)$ \\
\hline
\end{tabular}

Table 2: $Z\left(C_{m, n}\right)$ for $m \leq 11$ and odd $n$.

\begin{tabular}{|c|c|c|c|c|}
\hline \multirow{2}{*}{\multicolumn{2}{|c|}{$Z\left(C_{m, n}\right)$}} & \multicolumn{3}{|c|}{$n \bmod 6$} \\
\hline & & 1 & 3 & 5 \\
\hline \multirow{3}{*}{$m \bmod 3$} & 1 & $\bar{~} \overline{1}$ & $\overline{-2}$ & $\overline{1}$ \\
\hline & 2 & 1 & 1 & 1 \\
\hline & 3 & 1 & 1 & 1 \\
\hline
\end{tabular}

Further computational results appear in the work of van Eerten [11]. The grids under consideration are square, hexagonal, and triangular grids along with their dimer counterparts, and for all but the first family, the growth at $z=-1$ appears to be exponential.

\section{Acknowledgment}

This work was carried out at the Technical University of Berlin within the European Graduate Program "Combinatorics, Geometry, and Computation." I thank Günter Ziegler for his kind hospitality. I also thank Johan Thapper for pointing out an error in Table 3 in a previous version of the paper. 
Table 3: $Z\left(C_{m, n}\right)$ for $m \leq 11$ and even $n$.

\begin{tabular}{|c|c|c|c|c|c|c|c|c|c|c|c|c|c|}
\hline \multirow{2}{*}{\multicolumn{2}{|c|}{$Z\left(C_{m, n}\right)$}} & \multicolumn{12}{|c|}{$n$} \\
\hline & & 2 & $\overline{4}$ & 6 & 8 & 10 & 12 & 14 & 16 & 18 & 20 & 22 & 24 \\
\hline \multirow{11}{*}{$m$} & $\overline{\overline{11}}$ & $\bar{~} \overline{-1}$ & $\overline{-1-1}$ & $\overline{\overline{2}}$ & $\bar{~} \overline{-1}$ & $\overline{-1-1}$ & $\overline{\overline{2}}$ & $\overline{-\overline{-1}}$ & $\overline{-1-1}$ & $\overline{\overline{2}}$ & $\overline{-1-1}$ & $\bar{~} \overline{-1}$ & $\overline{\overline{2}}$ \\
\hline & 2 & -1 & $\overline{3}$ & -1 & 3 & -1 & 3 & -1 & 3 & -1 & $\overline{3}$ & -1 & 3 \\
\hline & 3 & 1 & $\overline{-3}$ & 1 & 5 & 1 & -3 & 1 & 5 & 1 & $\overline{-3}$ & 1 & 5 \\
\hline & 4 & 1 & 5 & 4 & 5 & 1 & 8 & 1 & 5 & 4 & 5 & 1 & 8 \\
\hline & 5 & -1 & -5 & -1 & 3 & $\overline{9}$ & -5 & -1 & $\overline{3}$ & -1 & 5 & -1 & $\overline{3}$ \\
\hline & 6 & -1 & 7 & -1 & 7 & -1 & 7 & 13 & 7 & -1 & 7 & -1 & 7 \\
\hline & 7 & 1 & -7 & 4 & 1 & 1 & 8 & 1 & 1 & 22 & -7 & 1 & 16 \\
\hline & 8 & 1 & 9 & 1 & 1 & 1 & 9 & 1 & 33 & 1 & 9 & 23 & 1 \\
\hline & 9 & -1 & -9 & -1 & -1 & -11 & -9 & 13 & -1 & -1 & 41 & -1 & -1 \\
\hline & 10 & -1 & 11 & 2 & 3 & -1 & 14 & -1 & 3 & 38 & 11 & -1 & 78 \\
\hline & 11 & 1 & -11 & 1 & -3 & 1 & -11 & 15 & 13 & 1 & -11 & 89 & -3 \\
\hline
\end{tabular}

\section{References}

[1] R. J. Baxter, Hard Hexagons: Exact Solution, J. Physics A 13 (1980), 1023-1030.

[2] N. G. de Bruijn, Algebraic theory of Penrose's non-periodic tilings of the plane, Math. Proc. A 84 (1981), 39-66.

[3] P. Fendley and K. Schoutens, Exact results for strongly-correlated fermions in 2+1 dimensions, Phys. Rev. Lett. 95 (2005), 046403.

[4] P. Fendley, K. Schoutens, and H. van Eerten, Hard squares with negative activity, J. Phys. A: Math. Gen. 38 (2005), no. 2, 315-322.

[5] R. Forman, Morse theory for cell complexes, Adv. Math. 134 (1998), 90-145.

[6] M. Fulmek and C. Krattenthaler, The number of rhombus tilings of a symmetric hexagon which contains a fixed rhombus on the symmetry axis, I, Ann. Combin. 2 (1998), 19-40.

[7] M. Fulmek and C. Krattenthaler, The number of rhombus tilings of a symmetric hexagon which contains a fixed rhombus on the symmetry axes, II, Europ. J. Combin. 21 (2000), 601-640. 2000.

[8] J. Jonsson, Certain homology cycles of the independence complex of grid graphs, Manuscript, 2005.

[9] D. Kozlov, Complexes of directed trees, J. Combin. Theory, Ser. A 88 (1999), no. 1, 112-122.

[10] R. Penrose, The rôle of aesthetics in pure and applied mathematical research, Bull. Inst. Math. Appl. 10 (1974), 266-271.

[11] H. van Eerten, Extensive ground state entropy in supersymmetric lattice models, preprint, 2005, cond-mat/0509581. 


\section{A The characteristic polynomial of $T_{m}$ for small $m$}

Fendley et al. [4] computed the characteristic polynomial $P_{m}(t)$ of the transfer matrix $T_{m}$ for $m \leq 15$. Since the size of $T_{m}$ grows exponentially, it is hard to compute $P_{m}(t)$ directly from $T_{m}$ for large $m$. Yet, by Theorem 3.4, we may instead compute $P_{m}(t)$ by counting rhombus tilings, which is a substantially easier task (indeed polynomial in $m$ ) thanks to Theorem 2.6.

Define $\tilde{P}_{m}(t)=P_{m}(t) / g_{m}(t)$, where $g_{m}(t)$ is defined as in Theorem 3.4. Using computer, we have calculated $\tilde{P}_{m}(t)$ for $m \leq 50$ :

$$
\begin{aligned}
& \tilde{P}_{1}= 1 \\
& \tilde{P}_{2}= t^{4}-1 \\
& \tilde{P}_{3}= 1 \\
& \tilde{P}_{4}=\left(t^{2}-1\right)^{2}\left(t^{4}-1\right) \\
& \tilde{P}_{5}=\left(t^{5}+1\right)^{2} \\
& \tilde{P}_{6}=\left(t^{4}-1\right)\left(t^{6}-1\right)^{3} . \\
& \tilde{P}_{7}=\left(t^{14}+1\right)^{2} . \\
& \tilde{P}_{8}=\left(t^{2}-1\right)^{2}\left(t^{4}-1\right)\left(t^{10}-1\right)^{4} \\
& \tilde{P}_{9}=\left(t^{9}-1\right)^{4}\left(t^{18}-1\right)^{2} \\
& \tilde{P}_{10}=\left(t^{4}-1\right)\left(t^{5}-1\right)^{2}\left(t^{8}-1\right)^{5}\left(t^{14}-1\right)^{5} . \\
& \tilde{P}_{16}=\left(t^{2}-1\right)^{2}\left(t^{4}-1\right)\left(t^{10}-1\right)^{4}\left(t^{14}-1\right)^{32}\left(t^{20}-1\right)^{48}\left(t^{26}-1\right)^{8}\left(t^{32}-1\right)^{10} \\
& \tilde{P}_{11}=\left(t^{55}-1\right)^{2}\left(t^{22}+1\right)^{4} . \\
& \tilde{P}_{12}=\left(t^{2}-1\right)^{2}\left(t^{4}-1\right)\left(t^{6}-1\right)^{3}\left(t^{12}-1\right)^{12}\left(t^{18}-1\right)^{6}\left(t^{24}-1\right)^{2} \\
& \tilde{P}_{13}=\left(t^{26}-1\right)^{4}\left(t^{91}-1\right)^{4}\left(t^{13}+1\right)^{4} . \\
& \tilde{P}_{14}=\left(t^{4}-1\right)\left(t^{10}-1\right)^{7}\left(t^{14}-1\right)^{2}\left(t^{16}-1\right)^{28}\left(t^{22}-1\right)^{7}\left(t^{28}-1\right)^{4}\left(t^{7}+1\right)^{4} \\
& \tilde{P}_{15}=\left(t^{15}-1\right)^{12}\left(t^{30}-1\right)^{6}\left(t^{45}-1\right)^{18}\left(t^{5}+1\right)^{2}\left(t^{30}+1\right)^{6} \\
& \tilde{P}^{6}\left(t^{136}-1\right)^{8}\left(t^{187}-1\right)^{10}\left(t^{34}+1\right)^{2}\left(t^{68}+1\right)^{4}
\end{aligned}
$$




$$
\begin{aligned}
& \tilde{P}_{18}=\left(t^{4}-1\right)\left(t^{6}-1\right)^{3}\left(t^{9}-1\right)^{8}\left(t^{12}-1\right)^{9}\left(t^{18}-1\right)^{101}\left(t^{24}-1\right)^{81}\left(t^{30}-1\right)^{9} \\
& \left(t^{36}-1\right)^{16}\left(t^{27}+1\right)^{36} \text {. } \\
& \tilde{P}_{19}=\left(t^{19}-1\right)^{4}\left(t^{38}-1\right)^{6}\left(t^{133}-1\right)^{4}\left(t^{190}-1\right)^{20}\left(t^{247}-1\right)^{14}\left(t^{38}+1\right)^{8}\left(t^{95}+1\right)^{10} \text {. } \\
& \tilde{P}_{20}=\left(t^{2}-1\right)^{2}\left(t^{4}-1\right)\left(t^{5}-1\right)^{2}\left(t^{8}-1\right)^{5}\left(t^{14}-1\right)^{5}\left(t^{16}-1\right)^{60}\left(t^{22}-1\right)^{250} \\
& \left(t^{28}-1\right)^{120}\left(t^{34}-1\right)^{10}\left(t^{40}-1\right)^{28}\left(t^{160}-1\right)^{4}\left(t^{220}-1\right)^{14} \text {. } \\
& \begin{aligned}
\tilde{P}_{21}= & \left(t^{21}-1\right)^{36}\left(t^{42}-1\right)^{26}\left(t^{63}-1\right)^{60}\left(t^{84}-1\right)^{144}\left(t^{105}-1\right)^{56}\left(t^{14}+1\right)^{2}\left(t^{21}+1\right)^{4} \\
& \left(t^{42}+1\right)^{18} .
\end{aligned} \\
& \tilde{P}_{22}=\left(t^{4}-1\right)\left(t^{14}-1\right)^{11}\left(t^{20}-1\right)^{275}\left(t^{22}-1\right)^{4}\left(t^{26}-1\right)^{539}\left(t^{32}-1\right)^{176}\left(t^{38}-1\right)^{11} \\
& \left(t^{44}-1\right)^{42}\left(t^{55}-1\right)^{14}\left(t^{110}-1\right)^{28}\left(t^{11}+1\right)^{8}\left(t^{143}+1\right)^{56} \text {. } \\
& \tilde{P}_{23}=\left(t^{46}-1\right)^{40}\left(t^{161}-1\right)^{28}\left(t^{184}-1\right)^{4}\left(t^{253}-1\right)^{78}\left(t^{322}-1\right)^{84}\left(t^{391}-1\right)^{24} \\
& \left(t^{23}+1\right)^{8}\left(t^{46}+1\right)^{10}\left(t^{92}+1\right)^{2} \text {. } \\
& \tilde{P}_{24}=\left(t^{2}-1\right)^{2}\left(t^{4}-1\right)\left(t^{6}-1\right)^{3}\left(t^{10}-1\right)^{4}\left(t^{12}-1\right)^{16}\left(t^{18}-1\right)^{114}\left(t^{24}-1\right)^{926} \\
& \left(t^{30}-1\right)^{1044}\left(t^{36}-1\right)^{240}\left(t^{42}-1\right)^{12}\left(t^{48}-1\right)^{68}\left(t^{72}-1\right)^{18}\left(t^{96}-1\right)^{168} \\
& \left(t^{120}-1\right)^{150} \text {. } \\
& \tilde{P}_{25}=\left(t^{25}-1\right)^{4}\left(t^{50}-1\right)^{10}\left(t^{125}-1\right)^{10}\left(t^{250}-1\right)^{34}\left(t^{325}-1\right)^{220}\left(t^{400}-1\right)^{160} \\
& \left(t^{475}-1\right)^{30}\left(t^{5}+1\right)^{2}\left(t^{50}+1\right)^{84}\left(t^{125}+1\right)^{2}\left(t^{200}+1\right)^{16} \text {. } \\
& \tilde{P}_{26}=\left(t^{4}-1\right)\left(t^{13}-1\right)^{12}\left(t^{16}-1\right)^{13}\left(t^{22}-1\right)^{637}\left(t^{26}-1\right)^{4}\left(t^{28}-1\right)^{2548}\left(t^{34}-1\right)^{1872} \\
& \left(t^{40}-1\right)^{325}\left(t^{46}-1\right)^{13}\left(t^{52}-1\right)^{110}\left(t^{91}-1\right)^{28}\left(t^{182}-1\right)^{280}\left(t^{286}-1\right)^{2} \\
& \left(t^{143}+1\right)^{80}\left(t^{221}+1\right)^{168} \text {. } \\
& \tilde{P}_{27}=\left(t^{9}-1\right)^{4}\left(t^{18}-1\right)^{2}\left(t^{27}-1\right)^{124}\left(t^{36}-1\right)^{216}\left(t^{45}-1\right)^{540}\left(t^{54}-1\right)^{206}\left(t^{81}-1\right)^{18} \\
& \left(t^{108}-1\right)^{448}\left(t^{135}-1\right)^{1400}\left(t^{162}-1\right)^{720}\left(t^{189}-1\right)^{110}\left(t^{18}+1\right)^{54}\left(t^{54}+1\right)^{14} \\
& \left(t^{81}+1\right)^{180} \text {. } \\
& \tilde{P}_{28}=\left(t^{2}-1\right)^{2}\left(t^{4}-1\right)\left(t^{7}-1\right)^{4}\left(t^{10}-1\right)^{7}\left(t^{14}-1\right)^{10}\left(t^{16}-1\right)^{28}\left(t^{20}-1\right)^{168} \\
& \left(t^{22}-1\right)^{7}\left(t^{26}-1\right)^{2744}\left(t^{28}-1\right)^{4}\left(t^{32}-1\right)^{6160}\left(t^{38}-1\right)^{3150}\left(t^{44}-1\right)^{420} \\
& \left(t^{50}-1\right)^{14}\left(t^{56}-1\right)^{190}\left(t^{280}-1\right)^{10}\left(t^{364}-1\right)^{168}\left(t^{448}-1\right)^{336}\left(t^{532}-1\right)^{132} \\
& \left(t^{91}+1\right)^{40} \text {. } \\
& \tilde{P}_{29}=\left(t^{29}-1\right)^{4}\left(t^{58}-1\right)^{22}\left(t^{203}-1\right)^{30}\left(t^{290}-1\right)^{20}\left(t^{319}-1\right)^{66}\left(t^{406}-1\right)^{650} \\
& \left(t^{493}-1\right)^{1140}\left(t^{580}-1\right)^{400}\left(t^{667}-1\right)^{44}\left(t^{29}+1\right)^{4}\left(t^{58}+1\right)^{264}\left(t^{145}+1\right)^{10} \\
& \left(t^{203}+1\right)^{56} \text {. } \\
& \tilde{P}_{30}=\left(t^{4}-1\right)\left(t^{5}-1\right)^{2}\left(t^{6}-1\right)^{3}\left(t^{8}-1\right)^{5}\left(t^{14}-1\right)^{5}\left(t^{15}-1\right)^{24}\left(t^{18}-1\right)^{15} \\
& \left(t^{24}-1\right)^{1305}\left(t^{30}-1\right)^{9681}\left(t^{36}-1\right)^{13500}\left(t^{42}-1\right)^{5040}\left(t^{45}-1\right)^{918}\left(t^{48}-1\right)^{540} \\
& \left(t^{54}-1\right)^{15}\left(t^{60}-1\right)^{770}\left(t^{90}-1\right)^{3672}\left(t^{120}-1\right)^{18}\left(t^{150}-1\right)^{110}\left(t^{15}+1\right)^{12} \\
& \left(t^{75}+1\right)^{3360}\left(t^{105}+1\right)^{1188} \text {. }
\end{aligned}
$$




$$
\begin{aligned}
& \tilde{P}_{31}=\left(t^{62}-1\right)^{424}\left(t^{155}-1\right)^{2}\left(t^{248}-1\right)^{16}\left(t^{310}-1\right)^{6}\left(t^{341}-1\right)^{110}\left(t^{403}-1\right)^{408} \\
& \left(t^{496}-1\right)^{2000}\left(t^{589}-1\right)^{2244}\left(t^{682}-1\right)^{550}\left(t^{775}-1\right)^{52}\left(t^{31}+1\right)^{20}\left(t^{62}+1\right)^{58} \\
& \left(t^{124}+1\right)^{8}\left(t^{248}+1\right)^{168} \text {. } \\
& \tilde{P}_{32}=\left(t^{2}-1\right)^{2}\left(t^{4}-1\right)\left(t^{10}-1\right)^{4}\left(t^{14}-1\right)^{32}\left(t^{16}-1\right)^{20}\left(t^{20}-1\right)^{48}\left(t^{22}-1\right)^{256} \\
& \left(t^{26}-1\right)^{8}\left(t^{28}-1\right)^{7040}\left(t^{32}-1\right)^{10}\left(t^{34}-1\right)^{28224}\left(t^{40}-1\right)^{27200}\left(t^{46}-1\right)^{7744} \\
& \left(t^{52}-1\right)^{672}\left(t^{58}-1\right)^{16}\left(t^{64}-1\right)^{608}\left(t^{112}-1\right)^{2}\left(t^{224}-1\right)^{76}\left(t^{272}-1\right)^{220} \\
& \left(t^{352}-1\right)^{14}\left(t^{448}-1\right)^{420}\left(t^{544}-1\right)^{1584}\left(t^{640}-1\right)^{1320}\left(t^{736}-1\right)^{286} \text {. } \\
& \tilde{P}_{33}=\left(t^{33}-1\right)^{376}\left(t^{55}-1\right)^{2}\left(t^{66}-1\right)^{340}\left(t^{99}-1\right)^{546}\left(t^{132}-1\right)^{272}\left(t^{165}-1\right)^{5562} \\
& \left(t^{198}-1\right)^{15876}\left(t^{231}-1\right)^{12360}\left(t^{264}-1\right)^{2520}\left(t^{297}-1\right)^{180}\left(t^{22}+1\right)^{4}\left(t^{33}+1\right)^{12} \\
& \left(t^{66}+1\right)^{722}\left(t^{99}+1\right)^{594}\left(t^{132}+1\right)^{108} \text {. } \\
& \tilde{P}_{34}=\left(t^{4}-1\right)\left(t^{17}-1\right)^{16}\left(t^{20}-1\right)^{17}\left(t^{26}-1\right)^{2448}\left(t^{32}-1\right)^{29988}\left(t^{34}-1\right)^{8} \\
& \left(t^{38}-1\right)^{74052}\left(t^{44}-1\right)^{51425}\left(t^{50}-1\right)^{11492}\left(t^{56}-1\right)^{833}\left(t^{62}-1\right)^{17}\left(t^{68}-1\right)^{1098} \\
& \left(t^{136}-1\right)^{208}\left(t^{187}-1\right)^{110}\left(t^{272}-1\right)^{3360}\left(t^{374}-1\right)^{4664}\left(t^{442}-1\right)^{14}\left(t^{544}-1\right)^{176} \\
& \left(t^{646}-1\right)^{286}\left(t^{17}+1\right)^{4}\left(t^{85}+1\right)^{4}\left(t^{221}+1\right)^{280}\left(t^{323}+1\right)^{7920}\left(t^{425}+1\right)^{800} \text {. } \\
& \tilde{P}_{35}=\left(t^{35}-1\right)^{28}\left(t^{49}-1\right)^{10}\left(t^{70}-1\right)^{994}\left(t^{98}-1\right)^{30}\left(t^{119}-1\right)^{260}\left(t^{245}-1\right)^{28} \\
& \left(t^{350}-1\right)^{528}\left(t^{385}-1\right)^{8}\left(t^{490}-1\right)^{740}\left(t^{595}-1\right)^{6864}\left(t^{700}-1\right)^{12680} \\
& \left(t^{805}-1\right)^{7150}\left(t^{910}-1\right)^{1092}\left(t^{1015}-1\right)^{70}\left(t^{5}+1\right)^{2}\left(t^{14}+1\right)^{2}\left(t^{70}+1\right)^{416} \\
& \left(t^{175}+1\right)^{264}\left(t^{245}+1\right)^{60}\left(t^{350}+1\right)^{50}\left(t^{455}+1\right)^{182} \text {. } \\
& \tilde{P}_{36}=\left(t^{2}-1\right)^{2}\left(t^{4}-1\right)\left(t^{6}-1\right)^{3}\left(t^{9}-1\right)^{8}\left(t^{12}-1\right)^{21}\left(t^{18}-1\right)^{139}\left(t^{24}-1\right)^{443} \\
& \left(t^{27}-1\right)^{324}\left(t^{30}-1\right)^{16209}\left(t^{36}-1\right)^{106904}\left(t^{42}-1\right)^{176418}\left(t^{48}-1\right)^{91980} \\
& \left(t^{54}-1\right)^{24120}\left(t^{60}-1\right)^{1026}\left(t^{66}-1\right)^{18}\left(t^{72}-1\right)^{1940}\left(t^{144}-1\right)^{56}\left(t^{180}-1\right)^{2750} \\
& \left(t^{216}-1\right)^{17820}\left(t^{252}-1\right)^{26950}\left(t^{288}-1\right)^{12012}\left(t^{324}-1\right)^{1638}\left(t^{45}+1\right)^{1344} \\
& \left(t^{63}+1\right)^{8008} \text {. } \\
& \tilde{P}_{37}=\left(t^{37}-1\right)^{28}\left(t^{74}-1\right)^{1010}\left(t^{185}-1\right)^{52}\left(t^{259}-1\right)^{14}\left(t^{296}-1\right)^{168}\left(t^{407}-1\right)^{858} \\
& \left(t^{481}-1\right)^{148}\left(t^{518}-1\right)^{42}\left(t^{592}-1\right)^{4288}\left(t^{703}-1\right)^{21896}\left(t^{814}-1\right)^{27610} \\
& \left(t^{925}-1\right)^{11830}\left(t^{1036}-1\right)^{1568}\left(t^{1147}-1\right)^{80}\left(t^{37}+1\right)^{4}\left(t^{74}+1\right)^{1458}\left(t^{148}+1\right)^{84} \\
& \left(t^{296}+1\right)^{96}\left(t^{407}+1\right)^{550} \text {. } \\
& \tilde{P}_{38}=\left(t^{4}-1\right)\left(t^{19}-1\right)^{16}\left(t^{22}-1\right)^{19}\left(t^{28}-1\right)^{4275}\left(t^{34}-1\right)^{82764}\left(t^{38}-1\right)^{14} \\
& \left(t^{40}-1\right)^{331056}\left(t^{46}-1\right)^{388531}\left(t^{52}-1\right)^{157339}\left(t^{58}-1\right)^{23275}\left(t^{64}-1\right)^{1216} \\
& \left(t^{70}-1\right)^{19}\left(t^{76}-1\right)^{3420}\left(t^{95}-1\right)^{858}\left(t^{133}-1\right)^{44}\left(t^{152}-1\right)^{10}\left(t^{190}-1\right)^{1716} \\
& \left(t^{247}-1\right)^{2002}\left(t^{266}-1\right)^{440}\left(t^{380}-1\right)^{34320}\left(t^{494}-1\right)^{12012}\left(t^{532}-1\right)^{28} \\
& \left(t^{646}-1\right)^{624}\left(t^{760}-1\right)^{2000}\left(t^{874}-1\right)^{1430}\left(t^{19}+1\right)^{16} \\
& \left(t^{323}+1\right)^{9268}\left(t^{437}+1\right)^{37752}\left(t^{551}+1\right)^{1456} \text {. } \\
& \tilde{P}_{39}=\left(t^{26}-1\right)^{4}\left(t^{39}-1\right)^{1264}\left(t^{78}-1\right)^{2720}\left(t^{91}-1\right)^{4}\left(t^{117}-1\right)^{3420}\left(t^{156}-1\right)^{312} \\
& \left(t^{195}-1\right)^{5096}\left(t^{234}-1\right)^{57870}\left(t^{273}-1\right)^{183870}\left(t^{312}-1\right)^{168940}\left(t^{351}-1\right)^{56004} \\
& \left(t^{390}-1\right)^{5880}\left(t^{429}-1\right)^{272}\left(t^{13}+1\right)^{4}\left(t^{39}+1\right)^{42}\left(t^{78}+1\right)^{2312}\left(t^{117}+1\right)^{720} \\
& \left(t^{156}+1\right)^{6006} \text {. }
\end{aligned}
$$




$$
\begin{aligned}
\tilde{P}_{40}= & \left(t^{2}-1\right)^{2}\left(t^{4}-1\right)\left(t^{5}-1\right)^{2}\left(t^{8}-1\right)^{5}\left(t^{10}-1\right)^{4}\left(t^{14}-1\right)^{5}\left(t^{16}-1\right)^{60}\left(t^{20}-1\right)^{56} \\
& \left(t^{22}-1\right)^{250}\left(t^{26}-1\right)^{500}\left(t^{28}-1\right)^{120}\left(t^{32}-1\right)^{34000}\left(t^{34}-1\right)^{10}\left(t^{38}-1\right)^{348480} \\
& \left(t^{40}-1\right)^{28}\left(t^{44}-1\right)^{920080}\left(t^{50}-1\right)^{801600}\left(t^{56}-1\right)^{258720}\left(t^{62}-1\right)^{32000} \\
& \left(t^{68}-1\right)^{1440}\left(t^{74}-1\right)^{20}\left(t^{80}-1\right)^{5984}\left(t^{160}-1\right)^{4}\left(t^{220}-1\right)^{14}\left(t^{320}-1\right)^{544} \\
& \left(t^{380}-1\right)^{5460}\left(t^{440}-1\right)^{11004}\left(t^{500}-1\right)^{5720}\left(t^{520}-1\right)^{24}\left(t^{640}-1\right)^{1856} \\
& \left(t^{760}-1\right)^{18946}\left(t^{880}-1\right)^{47190}\left(t^{1000}-1\right)^{37180}\left(t^{1120}-1\right)^{10192}\left(t^{1240}-1\right)^{952}
\end{aligned}
$$

$$
\begin{aligned}
\tilde{P}_{41}= & \left(t^{41}-1\right)^{4}\left(t^{82}-1\right)^{4870}\left(t^{164}-1\right)^{80}\left(t^{205}-1\right)^{10}\left(t^{287}-1\right)^{30}\left(t^{410}-1\right)^{830} \\
& \left(t^{533}-1\right)^{1274}\left(t^{574}-1\right)^{190}\left(t^{697}-1\right)^{9614}\left(t^{820}-1\right)^{73088}\left(t^{943}-1\right)^{155610} \\
& \left(t^{1066}-1\right)^{107614}\left(t^{1189}-1\right)^{29016}\left(t^{1312}-1\right)^{2688}\left(t^{1435}-1\right)^{102}\left(t^{41}+1\right)^{58} \\
& \left(t^{82}+1\right)^{2936}\left(t^{205}+1\right)^{400}\left(t^{287}+1\right)^{4}\left(t^{410}+1\right)^{1320}\left(t^{533}+1\right)^{2860}\left(t^{656}+1\right)^{64} .
\end{aligned}
$$$$
\tilde{P}_{42}=\left(t^{4}-1\right)\left(t^{6}-1\right)^{3}\left(t^{10}-1\right)^{7}\left(t^{14}-1\right)^{2}\left(t^{16}-1\right)^{28}\left(t^{21}-1\right)^{92}\left(t^{22}-1\right)^{7}
$$$$
\left(t^{24}-1\right)^{21}\left(t^{28}-1\right)^{4}\left(t^{30}-1\right)^{7056}\left(t^{36}-1\right)^{205821}\left(t^{42}-1\right)^{1264571}
$$$$
\left(t^{48}-1\right)^{2337993}\left(t^{54}-1\right)^{1565109}\left(t^{60}-1\right)^{411600}\left(t^{63}-1\right)^{2844}\left(t^{66}-1\right)^{43155}
$$$$
\left(t^{72}-1\right)^{1701}\left(t^{78}-1\right)^{21}\left(t^{84}-1\right)^{17534}\left(t^{105}-1\right)^{840}\left(t^{126}-1\right)^{64638}
$$$$
\left(t^{168}-1\right)^{686400}\left(t^{210}-1\right)^{92550}\left(t^{252}-1\right)^{5400}\left(t^{294}-1\right)^{30030}\left(t^{336}-1\right)^{41184}
$$$$
\left(t^{378}-1\right)^{16200}\left(t^{7}+1\right)^{4}\left(t^{21}+1\right)^{36}\left(t^{63}+1\right)^{13728}\left(t^{105}+1\right)^{2160}\left(t^{147}+1\right)^{398076}
$$$$
\left(t^{189}+1\right)^{411840}\left(t^{231}+1\right)^{7344} \text {. }
$$

$$
\begin{aligned}
\tilde{P}_{43}= & \left(t^{43}-1\right)^{48}\left(t^{86}-1\right)^{4200}\left(t^{215}-1\right)^{1430}\left(t^{301}-1\right)^{1456}\left(t^{344}-1\right)^{88}\left(t^{473}-1\right)^{1498} \\
& \left(t^{559}-1\right)^{10}\left(t^{602}-1\right)^{4368}\left(t^{688}-1\right)^{2304}\left(t^{817}-1\right)^{50830}\left(t^{946}-1\right)^{240206} \\
& \left(t^{1075}-1\right)^{363896}\left(t^{1204}-1\right)^{197008}\left(t^{1333}-1\right)^{43384}\left(t^{1462}-1\right)^{3264}\left(t^{1591}-1\right)^{114} \\
& \left(t^{43}+1\right)^{16}\left(t^{86}+1\right)^{9744}\left(t^{172}+1\right)^{44}\left(t^{344}+1\right)^{88}\left(t^{473}+1\right)^{4290}\left(t^{602}+1\right)^{196} \\
& \left(t^{731}+1\right)^{408} .
\end{aligned}
$$

$$
\begin{aligned}
\tilde{P}_{44}= & \left(t^{2}-1\right)^{2}\left(t^{4}-1\right)\left(t^{11}-1\right)^{8}\left(t^{14}-1\right)^{11}\left(t^{20}-1\right)^{275}\left(t^{22}-1\right)^{88}\left(t^{26}-1\right)^{539} \\
& \left(t^{28}-1\right)^{660}\left(t^{32}-1\right)^{176}\left(t^{34}-1\right)^{66550}\left(t^{38}-1\right)^{11}\left(t^{40}-1\right)^{1012088}\left(t^{44}-1\right)^{42} \\
& \left(t^{46}-1\right)^{4048902}\left(t^{52}-1\right)^{5510736}\left(t^{55}-1\right)^{910}\left(t^{58}-1\right)^{2914912}\left(t^{64}-1\right)^{635712} \\
& \left(t^{70}-1\right)^{57222}\left(t^{76}-1\right)^{1980}\left(t^{82}-1\right)^{22}\left(t^{88}-1\right)^{18466}\left(t^{110}-1\right)^{1820} \\
& \left(t^{143}-1\right)^{19448}\left(t^{176}-1\right)^{264}\left(t^{220}-1\right)^{36400}\left(t^{286}-1\right)^{116688}\left(t^{308}-1\right)^{2} \\
& \left(t^{616}-1\right)^{30}\left(t^{748}-1\right)^{3456}\left(t^{880}-1\right)^{53200}\left(t^{1012}-1\right)^{205442}\left(t^{1144}-1\right)^{260260} \\
& \left(t^{1276}-1\right)^{123760}\left(t^{1408}-1\right)^{22848}\left(t^{1540}-1\right)^{1550}\left(t^{187}+1\right)^{2352}\left(t^{253}+1\right)^{128128} \\
& \left(t^{319}+1\right)^{38896} .
\end{aligned}
$$

$$
\begin{aligned}
\tilde{P}_{45}= & \left(t^{9}-1\right)^{4}\left(t^{15}-1\right)^{66}\left(t^{18}-1\right)^{2}\left(t^{27}-1\right)^{900}\left(t^{30}-1\right)^{1356}\left(t^{45}-1\right)^{7722} \\
& \left(t^{54}-1\right)^{6300}\left(t^{63}-1\right)^{77000}\left(t^{72}-1\right)^{184800}\left(t^{75}-1\right)^{72144}\left(t^{81}-1\right)^{119340} \\
& \left(t^{90}-1\right)^{61152}\left(t^{105}-1\right)^{486486}\left(t^{120}-1\right)^{1441440}\left(t^{135}-1\right)^{1474200} \\
& \left(t^{150}-1\right)^{501228}\left(t^{165}-1\right)^{69768}\left(t^{180}-1\right)^{16016}\left(t^{225}-1\right)^{660}\left(t^{270}-1\right)^{41820} \\
& \left(t^{315}-1\right)^{490762}\left(t^{360}-1\right)^{1601600}\left(t^{405}-1\right)^{1873872}\left(t^{450}-1\right)^{862400} \\
& \left(t^{495}-1\right)^{166464}\left(t^{540}-1\right)^{12960}\left(t^{585}-1\right)^{380}\left(t^{5}+1\right)^{2}\left(t^{30}+1\right)^{6}\left(t^{36}+1\right)^{1680} \\
& \left(t^{45}+1\right)^{4}\left(t^{60}+1\right)^{61776}\left(t^{90}+1\right)^{6390}\left(t^{135}+1\right)^{1842}\left(t^{225}+1\right)^{7840}
\end{aligned}
$$




$$
\begin{aligned}
& \tilde{P}_{46}=\left(t^{4}-1\right)\left(t^{23}-1\right)^{88}\left(t^{26}-1\right)^{23}\left(t^{32}-1\right)^{11132}\left(t^{38}-1\right)^{470327}\left(t^{44}-1\right)^{4232943} \\
& \left(t^{46}-1\right)^{50}\left(t^{50}-1\right)^{11758175}\left(t^{56}-1\right)^{12189632}\left(t^{62}-1\right)^{5211248}\left(t^{68}-1\right)^{957168} \\
& \left(t^{74}-1\right)^{74727}\left(t^{80}-1\right)^{2300}\left(t^{86}-1\right)^{23}\left(t^{92}-1\right)^{32720}\left(t^{161}-1\right)^{28}\left(t^{184}-1\right)^{64} \\
& \left(t^{253}-1\right)^{10098}\left(t^{322}-1\right)^{34020}\left(t^{368}-1\right)^{1040}\left(t^{391}-1\right)^{7752}\left(t^{506}-1\right)^{428120} \\
& \left(t^{644}-1\right)^{1089088}\left(t^{736}-1\right)^{84}\left(t^{782}-1\right)^{62016}\left(t^{874}-1\right)^{4622}\left(t^{1012}-1\right)^{40040} \\
& \left(t^{1150}-1\right)^{91936}\left(t^{1288}-1\right)^{68068}\left(t^{1426}-1\right)^{16796}\left(t^{23}+1\right)^{20}\left(t^{115}+1\right)^{5720} \\
& \left(t^{437}+1\right)^{48528}\left(t^{575}+1\right)^{1149712}\left(t^{713}+1\right)^{424320}\left(t^{851}+1\right)^{3876} \text {. } \\
& \tilde{P}_{47}=\left(t^{47}-1\right)^{48}\left(t^{94}-1\right)^{10632}\left(t^{188}-1\right)^{424}\left(t^{235}-1\right)^{266}\left(t^{329}-1\right)^{104}\left(t^{470}-1\right)^{952} \\
& \left(t^{611}-1\right)^{20020}\left(t^{658}-1\right)^{10}\left(t^{752}-1\right)^{320}\left(t^{799}-1\right)^{3868}\left(t^{893}-1\right)^{570} \\
& \left(t^{940}-1\right)^{117648}\left(t^{1081}-1\right)^{843030}\left(t^{1222}-1\right)^{1894412}\left(t^{1363}-1\right)^{1644240} \\
& \left(t^{1504}-1\right)^{580416}\left(t^{1645}-1\right)^{90124}\left(t^{1786}-1\right)^{5130}\left(t^{1927}-1\right)^{140}\left(t^{47}+1\right)^{60} \\
& \left(t^{94}+1\right)^{33626}\left(t^{235}+1\right)^{80}\left(t^{376}+1\right)^{32}\left(t^{470}+1\right)^{2010}\left(t^{611}+1\right)^{7852} \\
& \left(t^{752}+1\right)^{11424} \text {. } \\
& \tilde{P}_{48}=\left(t^{2}-1\right)^{2}\left(t^{4}-1\right)\left(t^{6}-1\right)^{3}\left(t^{10}-1\right)^{4}\left(t^{12}-1\right)^{16}\left(t^{14}-1\right)^{32}\left(t^{18}-1\right)^{114} \\
& \left(t^{20}-1\right)^{48}\left(t^{24}-1\right)^{1062}\left(t^{26}-1\right)^{8}\left(t^{30}-1\right)^{1908}\left(t^{32}-1\right)^{10}\left(t^{36}-1\right)^{122880} \\
& \left(t^{42}-1\right)^{2672004}\left(t^{48}-1\right)^{15531608}\left(t^{54}-1\right)^{31409664}\left(t^{60}-1\right)^{25527024} \\
& \left(t^{66}-1\right)^{8989056}\left(t^{72}-1\right)^{1456074}\left(t^{78}-1\right)^{96264}\left(t^{84}-1\right)^{2856}\left(t^{90}-1\right)^{24} \\
& \left(t^{96}-1\right)^{59042}\left(t^{112}-1\right)^{2}\left(t^{120}-1\right)^{150}\left(t^{144}-1\right)^{11790}\left(t^{168}-1\right)^{166320} \\
& \left(t^{192}-1\right)^{884962}\left(t^{216}-1\right)^{1432080}\left(t^{240}-1\right)^{831410}\left(t^{264}-1\right)^{167960} \\
& \left(t^{288}-1\right)^{18000}\left(t^{336}-1\right)^{406296}\left(t^{384}-1\right)^{2312320}\left(t^{432}-1\right)^{4445856} \\
& \left(t^{480}-1\right)^{3341520}\left(t^{528}-1\right)^{1054272}\left(t^{576}-1\right)^{139482}\left(t^{624}-1\right)^{7182} \text {. } \\
& \tilde{P}_{49}=\left(t^{35}-1\right)^{14}\left(t^{49}-1\right)^{8}\left(t^{77}-1\right)^{714}\left(t^{98}-1\right)^{58784}\left(t^{133}-1\right)^{210}\left(t^{154}-1\right)^{3570} \\
& \left(t^{175}-1\right)^{10850}\left(t^{245}-1\right)^{3536}\left(t^{343}-1\right)^{9724}\left(t^{392}-1\right)^{4}\left(t^{539}-1\right)^{6006} \\
& \left(t^{686}-1\right)^{29452}\left(t^{784}-1\right)^{344}\left(t^{833}-1\right)^{4896}\left(t^{931}-1\right)^{36322}\left(t^{1078}-1\right)^{599242} \\
& \left(t^{1225}-1\right)^{2826356}\left(t^{1372}-1\right)^{4706464}\left(t^{1519}-1\right)^{3230136}\left(t^{1666}-1\right)^{943296} \\
& \left(t^{1813}-1\right)^{125970}\left(t^{1960}-1\right)^{6600}\left(t^{2107}-1\right)^{154}\left(t^{14}+1\right)^{2}\left(t^{49}+1\right)^{168} \\
& \left(t^{98}+1\right)^{19852}\left(t^{196}+1\right)^{2}\left(t^{343}+1\right)^{28}\left(t^{392}+1\right)^{44}\left(t^{539}+1\right)^{4080}\left(t^{686}+1\right)^{20384} \\
& \left(t^{833}+1\right)^{19380}\left(t^{980}+1\right)^{100} \text {. } \\
& \tilde{P}_{50}=\left(t^{4}-1\right)\left(t^{5}-1\right)^{2}\left(t^{8}-1\right)^{5}\left(t^{14}-1\right)^{5}\left(t^{25}-1\right)^{24}\left(t^{28}-1\right)^{25}\left(t^{34}-1\right)^{16900} \\
& \left(t^{40}-1\right)^{1002000}\left(t^{46}-1\right)^{12780625}\left(t^{50}-1\right)^{94}\left(t^{52}-1\right)^{51122500}\left(t^{58}-1\right)^{78145600} \\
& \left(t^{64}-1\right)^{50979600}\left(t^{70}-1\right)^{15023375}\left(t^{76}-1\right)^{2030625}\left(t^{82}-1\right)^{122500}\left(t^{88}-1\right)^{3025} \\
& \left(t^{94}-1\right)^{25}\left(t^{100}-1\right)^{104198}\left(t^{125}-1\right)^{44}\left(t^{200}-1\right)^{16}\left(t^{250}-1\right)^{3066} \\
& \left(t^{325}-1\right)^{35780}\left(t^{400}-1\right)^{14272}\left(t^{475}-1\right)^{570}\left(t^{500}-1\right)^{97456}\left(t^{650}-1\right)^{4899360} \\
& \left(t^{800}-1\right)^{4275840}\left(t^{850}-1\right)^{132}\left(t^{950}-1\right)^{128592}\left(t^{1000}-1\right)^{10570}\left(t^{1150}-1\right)^{136250} \\
& \left(t^{1300}-1\right)^{482664}\left(t^{1450}-1\right)^{587860}\left(t^{1600}-1\right)^{268736}\left(t^{1750}-1\right)^{45220}\left(t^{25}+1\right)^{168} \\
& \left(t^{125}+1\right)^{204}\left(t^{425}+1\right)^{1540}\left(t^{575}+1\right)^{1267200}\left(t^{725}+1\right)^{7117832}\left(t^{875}+1\right)^{1129548} \\
& \left(t^{1025}+1\right)^{5852} \text {. }
\end{aligned}
$$

